\title{
BASIS PROPERTIES OF THE HAAR SYSTEM IN LIMITING BESOV SPACES
}

\author{
GUSTAVO GARRIGÓS ANDREAS SEEGER TINO ULLRICH
}

\begin{abstract}
We study Schauder basis properties for the Haar system in Besov spaces $B_{p, q}^{s}\left(\mathbb{R}^{d}\right)$. We give a complete description of the limiting cases, obtaining various positive results for $q \leq \min \{1, p\}$, and providing new counterexamples in other situations. The study is based on suitable estimates of the dyadic averaging operators $\mathbb{E}_{N}$; in particular we find asymptotically optimal growth rates for the norms of these operators in global and local situations.
\end{abstract}

\section{INTRODUCTION}

The purpose of this paper is to complete the study of the basis properties of the (inhomogeneous) Haar system in the scale of Besov spaces $B_{p, q}^{s}\left(\mathbb{R}^{d}\right)$. In view of previous results, only the endpoint cases are of interest. This is a companion to the forthcoming paper [6], in which the authors consider the same endpoint questions for the Triebel-Lizorkin spaces. The outcomes for Besov and for Triebel-Lizorkin spaces, in both non-endpoint situations $([14,19,11,12,4])$ and endpoint situations, are markedly different.

To state the results, we first set some basic notation. Consider the one variable functions $h^{(0)}=\mathbb{1}_{[0,1)}$ and $h^{(1)}=\mathbb{1}_{[0,1 / 2)}-\mathbb{1}_{[1 / 2,1)}$. For every $\epsilon=\left(\epsilon_{1}, \ldots, \epsilon_{d}\right) \in\{0,1\}^{d}$, for $k \in \mathbb{N}_{0}$ and $\mu=\left(\mu_{1}, \ldots, \mu_{d}\right) \in \mathbb{Z}^{d}$ one defines

$$
h_{k, \mu}^{\epsilon}(x)=\prod_{i=1}^{d} h^{\left(\epsilon_{i}\right)}\left(2^{k} x_{i}-\mu_{i}\right), \quad x=\left(x_{1}, \ldots, x_{d}\right) \in \mathbb{R}^{d} .
$$

Denoting $\Upsilon=\{0,1\}^{d} \backslash\{\overrightarrow{0}\}$, the Haar system is given as

$$
\mathcal{H}_{d}=\left\{h_{0, \mu}^{\overrightarrow{0}}\right\}_{\mu \in \mathbb{Z}^{d}} \cup\left\{h_{k, \mu}^{\epsilon}: k \in \mathbb{N}_{0}, \mu \in \mathbb{Z}^{d}, \epsilon \in \Upsilon\right\} .
$$

We refer to $2^{k}$ as the Haar frequency of $h_{k, \mu}^{\epsilon}$. We consider an enumeration $\mathcal{U}=\left\{u_{n}\right\}_{n=1}^{\infty}$ of the Haar system, and write $u_{n}=h_{k(n), \mu(n)}^{\epsilon(n)}$ for the corresponding frequency and position parameters $k(n), \mu(n)$.

2010 Mathematics Subject Classification. 46E35, 46B15, 42C40.

Key words and phrases. Schauder basis, basic sequence, unconditional basis, local Schauder basis, dyadic averaging operators, Haar system, Besov and Triebel-Lizorkin spaces. 
Given $R \in \mathbb{N}$, the partial sum operator $S_{R} \equiv S_{R}^{\mathcal{U}}$ is defined as the projection onto $\operatorname{span}\left\{u_{1}, \ldots, u_{R}\right\}$, that is

$$
S_{R}^{\mathcal{U}} f=\sum_{n=1}^{R} u_{n}^{*}(f) u_{n},
$$

where for $u_{n}=h_{k(n), \mu(n)}^{\epsilon(n)}$ the linear functional $u_{n}^{*}$ is defined by

$$
u_{n}^{*}(f)=2^{k(n) d}\left\langle f, h_{k(n), \mu(n)}^{\epsilon(n)}\right\rangle,
$$

at least when $f \in L_{\text {loc }}^{1}\left(\mathbb{R}^{d}\right)$. Below we shall only consider Besov spaces so that $u_{n} \in B_{p, q}^{s}$ and $u_{n}^{*}$ extends to an element of $\left(B_{p, q}^{s}\right)^{*}$ for all $n \in \mathbb{N}$, so that (2) will actually have a meaning for all $f \in B_{p, q}^{s}$.

We say that $\mathcal{U}$ is a Schauder basis of $B_{p, q}^{s}\left(\mathbb{R}^{d}\right)$ if

$$
\lim _{R \rightarrow \infty}\left\|S_{R}^{\mathcal{U}} f-f\right\|_{B_{p, q}^{s}}=0
$$

holds for every $f \in B_{p, q}^{s}$. We say that $\mathcal{U}$ is a basic sequence if (3) holds for every $f$ in the $B_{p, q}^{s}$-closure of span $\mathcal{H}_{d}$. Finally, we say that $\mathcal{H}_{d}$ is an unconditional basis of $B_{p, q}^{s}$ if every enumeration $\mathcal{U}$ is a Schauder basis.

The above basis properties are related with the uniform bound

$$
C_{\mathcal{U}}:=\sup _{R \geq 1}\left\|S_{R}^{\mathcal{U}}\right\|_{B_{p, q}^{s} \rightarrow B_{p, q}^{s}}<\infty .
$$

Indeed, one has

$$
\left\|S_{R}^{\mathcal{U}} f-f\right\|_{B_{p, q}^{s}} \lesssim\left(C_{\mathcal{U}}+1\right)\|f-h\|_{B_{p, q}^{s}}+\left\|S_{R}^{\mathcal{U}} h-h\right\|_{B_{p, q}^{s},}, \quad h \in \operatorname{span} \mathcal{H}_{d} .
$$

Thus, (44) implies that $\mathcal{U}$ is a basic sequence in $B_{p, q}^{s}$. If $\operatorname{span}\left(\mathcal{H}_{d}\right)$ is dense in $B_{p, q}^{s}$, then $\mathcal{U}$ is a Schauder basis if and only if (44) holds. If in addition the bound in (4) does not depend on the enumeration $\mathcal{U}$ then $\mathcal{H}_{d}$ is an unconditional basis of $B_{p, q}^{s}$. By the uniform boundedness principle such a uniform estimate is also necessary for unconditionality. This is well-known for Banach spaces, and a proof for quasi-Banach spaces can be found in [1.

We consider the full range of indices $s \in \mathbb{R}$ and $0<p, q \leq \infty$. When $p=\infty$ or $q=\infty$ the space $B_{p, q}^{s}$ is not separable, but in those cases one may consider the Schauder basis property in the $B_{p, q}^{s}$-closure of the Schwartz class $\mathcal{S}$, which we will denote $b_{p, q}^{s}$ (as in [10, Def 1.1.3]).

The pentagon $\mathfrak{P}$ depicted in Figure 1 shows the natural index region for these problems. More precisely, Triebel showed in [14, 19] that, for all $q<\infty$, the Haar system $\mathcal{H}_{d}$ is an unconditional basis of $B_{p, q}^{s}\left(\mathbb{R}^{d}\right)$ for the $(1 / p, s)$ parameters in the interior of the pentagon $\mathfrak{P}$; i.e. those satisfying one of the conditions (i), (ii) in Theorem 1.1. He also showed that for the parameters in the complement of the closure of $\mathfrak{P}$ the Haar system does not form a basis. Except for a few trivial cases, the behavior at the points 

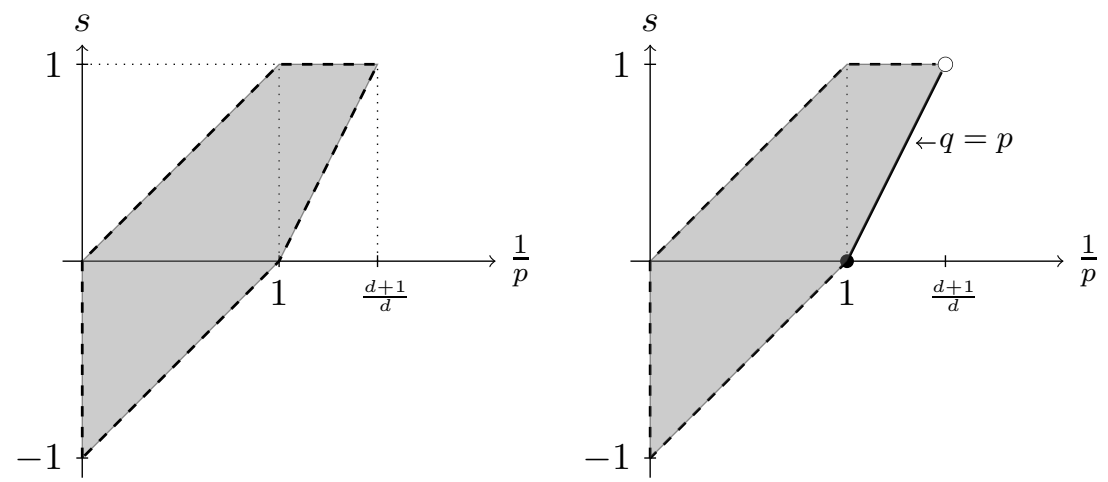

Figure 1. Parameter domain $\mathfrak{P}$ for $\mathcal{H}_{d}$ in $B_{p, q}^{s}\left(\mathbb{R}^{d}\right)$. The left figure shows the region of unconditionality, and right figure the region for the Schauder basis property.

$(1 / p, s)$ lying in the boundary of $\mathfrak{P}$ seems to be unexplored; see however the separate work [8] and Remark 1.7 below.

In this paper we attempt to fill these gaps, by giving an answer, positive or negative, depending on the secondary index $q$. Moreover, in some cases, the negative answer is replaced by slightly weaker properties, such as the local Schauder basis, or basic sequence properties.

We begin by stating complete results about unconditionality, which contain new negative cases compared to [14, 19]. We remark that the corresponding results in Triebel-Lizorkin spaces are much more restrictive, see the discussion in [19, Remark 2.2.10] and the counterexamples in [11].

Theorem 1.1. Let $0<p, q \leq \infty$ and $s \in \mathbb{R}$. Then, $\mathcal{H}_{d}$ is an unconditional basis of $B_{p, q}^{s}\left(\mathbb{R}^{d}\right)$ if and only if one of the following two conditions is satisfied.

$$
1 \leq p<\infty, \quad-1+\frac{1}{p}<s<\frac{1}{p}, \quad 0<q<\infty .
$$

(ii) $\frac{d}{d+1}<p<1, \quad d\left(\frac{1}{p}-1\right)<s<1, \quad 0<q<\infty$.

The region (i)-(ii) is shown in the left of Figure 1, In the next results we shall be concerned with the endpoint behavior when we drop unconditionality. To do so we must single out specific enumerations of the Haar system, labeled 'admissible', or 'strongly admissible'.

Definition 1.2. (i) An enumeration $\mathcal{U}$ is said to be admissible if there is a constant $b \in \mathbb{N}$ with the following property: for each cube $I_{\nu}=\nu+[0,1]^{d}, \nu \in$ $\mathbb{Z}^{d}$, if $u_{n}$ and $u_{n^{\prime}}$ are both supported in $I_{\nu}$ and $\left|\operatorname{supp}\left(u_{n}\right)\right| \geq 2^{b d}\left|\operatorname{supp}\left(u_{n^{\prime}}\right)\right|$, then necessarily $n<n^{\prime}$. 
(ii) An enumeration $\mathcal{U}$ is strongly admissible if there is a constant $b \in \mathbb{N}$ with the following property: for each cube $I_{\nu}, \nu \in \mathbb{Z}^{d}$, if $I_{\nu}^{* *}$ denotes the fivefold dilated cube with respect to its center, and if $u_{n}$ and $u_{n^{\prime}}$ are supported in $I_{\nu}^{* *}$ with $\left|\operatorname{supp}\left(u_{n}\right)\right| \geq 2^{b d}\left|\operatorname{supp}\left(u_{n^{\prime}}\right)\right|$ then necessarily $n<n^{\prime}$.

The notion in (i) was used in [4 for the case $b=1$, but the results stated in that paper continue to hold with this slightly more general definition.

The stronger notion in (ii) turns out to be more appropriate in the endpoint cases, for which the characteristic functions of cubes may not be pointwise multipliers; cf. [10. Loosely speaking, in a strongly admissible enumeration if a Haar frequency $2^{k}$ shows up at step $n$ (i.e. if $u_{n}=h_{k, \mu}^{\varepsilon}$ for some $k \in \mathbb{N}_{0}$ ) then all Haar functions with Haar frequency $\leq 2^{k-b}$ which are 'nearby' (in a well defined sense) have already been counted before step $n$. We refer to $\$ 11$ for concrete examples.

Finally, we remark that the above distinction is void for the classical Haar system in the unit cube, $\mathcal{H}\left([0,1)^{d}\right)$, where admissibility is a straightforward property; for $b=1$ it means that $n<n^{\prime}$ implies $\left|\operatorname{supp}\left(u_{n}\right)\right| \geq\left|\operatorname{supp}\left(u_{n^{\prime}}\right)\right|$ (and could be slightly weakened if $b \geq 2$ ). The typical example is the lexicographic ordering.

We now formulate a theorem involving all strongly admissible enumerations of the Haar system. A positive endpoint result is obtained for $B_{p, p}^{s}$ when $s=d / p-d$ and $\frac{d}{d+1}<p \leq 1$. Also new negative results are obtained for suitable strongly admissible enumerations; see the right of Figure 1.

Theorem 1.3. Let $0<p, q \leq \infty$ and $s \in \mathbb{R}$. Then, the following statements are equivalent, i.e. (a) $\Longleftrightarrow(b)$ :

(a) Every strongly admissible enumeration of $\mathcal{H}_{d}$ is a Schauder basis of $B_{p, q}^{s}\left(\mathbb{R}^{d}\right)$.

(b) One of the following three conditions is satisfied:

$$
\begin{array}{ll}
\text { (i) } & 1 \leq p<\infty, \quad \frac{1}{p}-1<s<\frac{1}{p}, \quad 0<q<\infty, \\
\text { (ii) } \quad \frac{d}{d+1}<p<1, \quad \frac{d}{p}-d<s<1, \quad 0<q<\infty, \\
\text { (iii) } \frac{d}{d+1}<p \leq 1, \quad s=\frac{d}{p}-d, \quad q=p .
\end{array}
$$

Next we explore various weaker properties at the boundary of $\mathfrak{P}$. We say that an enumeration $\mathcal{U}$ satisfies the local Schauder basis property for $B_{p, q}^{s}$ if

$$
\left\|\left(S_{R}^{\mathcal{U}} f-f\right) \chi\right\|_{B_{p, q}^{s}} \rightarrow 0
$$

for all $f \in B_{p, q}^{s}\left(\mathbb{R}^{d}\right)$ and all $\chi \in C_{c}^{\infty}\left(\mathbb{R}^{d}\right)$. This implies that the basis expansion holds, $g=\sum_{n=1}^{\infty} u_{n}^{*}(g) u_{n}$ in $B_{p, q}^{s}$, for all compactly supported $g \in B_{p, q}^{s}\left(\mathbb{R}^{d}\right)$. Similarly we say that $\mathcal{U}$ satisfies the local basic sequence property in $B_{p, q}^{s}\left(\mathbb{R}^{d}\right)$ when (5) holds for all $f \in{\overline{\operatorname{span} \mathcal{H}_{d}}}^{B_{p, q}^{s}}$ and all $\chi \in$ 
$C_{c}^{\infty}\left(\mathbb{R}^{d}\right)$. The next theorem and Figure 2 show the region of validity for the first of these properties.

Theorem 1.4. Let $0<p, q \leq \infty$ and $s \in \mathbb{R}$. Then, the following statements are equivalent, i.e. (a) $\Longleftrightarrow(b)$ :

(a) Every strongly admissible enumeration of the Haar system $\mathcal{H}_{d}$ satisfies the local Schauder basis property for $B_{p, q}^{s}\left(\mathbb{R}^{d}\right)$.

(b) One of the following four conditions is satisfied:

(i) $1 \leq p<\infty, \quad-1+\frac{1}{p}<s<\frac{1}{p}, \quad 0<q<\infty$,

(ii) $1 \leq p<\infty, \quad s=-1+\frac{1}{p}, \quad 0<q \leq 1$,

(iii) $\frac{d}{d+1}<p<1, \quad \frac{d}{p}-d<s<1, \quad 0<q<\infty$,

(iv) $\quad \frac{d}{d+1}<p<1, \quad s=\frac{d}{p}-d, \quad 0<q \leq p$.

We remark that these local properties can be given slightly stronger statements using the Bourdaud definitions $\left(B_{p, q}^{s}\right)_{\ell^{p}}$ of localized Besov spaces; see $\oint 9.2$ below.

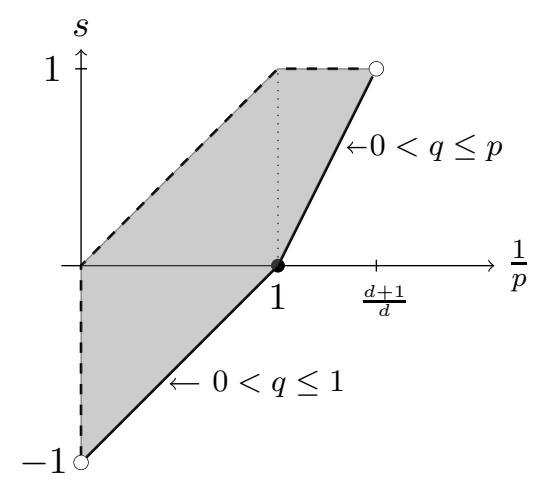

FiguRE 2. Region for the local Schauder basis property in the spaces $B_{p, q}^{s}\left(\mathbb{R}^{d}\right)$, depending on the value of $q \in(0, \infty)$.

Remark 1.5. Strong admissibility may be replaced by admissibility (for the positive results in Theorems 1.3 and 1.4$)$ in the cases where $\mathbb{1}_{[0,1]^{d}}$ is a pointwise multiplier of $B_{p, q}^{s}\left(\mathbb{R}^{d}\right)$. By [16, $\left.\S 2.8 .7\right]$ or [10, $\left.\S 4.6 .3\right]$, the latter holds when

$$
\max \left\{d\left(\frac{1}{p}-1\right), \frac{1}{p}-1\right\}<s<\frac{1}{p},
$$

so it applies to the interior points of $\mathfrak{P}$. It also applies in other positive results (such as the local basic sequence property, which will follow from Theorem 1.8) in the case $s=1, \frac{d}{d+1}<p<1$ corresponding to the interior of the horizontal edge of $\mathfrak{P}$. 
Remark 1.6. A similar statement to Theorem 1.4 holds for $\mathcal{H}\left(\mathbb{T}^{d}\right)$, the Haar system in the torus in the standard lexicographic enumeration. Namely, it is a Schauder basis on $B_{p, q}^{s}\left(\mathbb{T}^{d}\right)$ if and only if one of the conditions (i), (ii), (iii), (iv) in Theorem 1.4 are satisfied. Moreover, in the range (6) the class of $C^{\infty}$-functions with compact support in $(0,1)^{d}$ are dense in $B_{p, q}^{s}\left((0,1)^{d}\right)$ (see [18, §3.2]) and thus it is easy to see that the Schauder basis problem for the Haar systems on $\mathbb{T}^{d}$ and on $(0,1)^{d}$ are equivalent in this range. So far this observation does not apply to the cases corresponding to the non-horizontal edges of $\mathfrak{P}$ in higher dimensions, however see Franke's better result [3, $\S 4.6]$ for the interval $(0,1)$.

Remark 1.7. (i) In a classical work [7, P. Oswald considered, for $0<p<1$, the Schauder basis property (including some endpoint results) for a class of Besov spaces on the interval, $\mathcal{B}_{p, q,(1)}^{s}(I)$, defined by first order differences. In these classes, which in general differ from $B_{p, q}^{s}$, one has a positive answer in the larger region $1 / p-1<s<1 / p$ (in particular, for some $s \geq 1$ ); see [7, Theorem 3].

(ii) In a very recent separate study [8], Oswald pursued further these questions for both, the class $\mathcal{B}_{p, q,(1)}^{s}\left(I^{d}\right)$ and the standard Besov spaces $B_{p, q}^{s}\left((0,1)^{d}\right)$. He obtained analogs of the positive results in (ii)-(iv) of Theorem 1.4, and presented similar counterexamples as ours for the case $s=d / p-d$. Contrary to what is stated in that paper, these local results do not transfer to the spaces on $\mathbb{R}^{d}$ by simply enumerating the Haar system, as one may see from Theorem 1.3 above and the specific example in Proposition 12.1.

Dyadic averaging operators. A crucial tool in our analysis will be the dyadic averaging operator $\mathbb{E}_{N}$, defined as the conditional expectation with respect to the $\sigma$-algebra generated by the set $\mathcal{D}_{N}$ of all dyadic cubes of length $2^{-N}$. That is, setting

$$
I_{N, \mu}=2^{-N}\left(\mu+[0,1)^{d}\right), \quad \mu \in \mathbb{Z}^{d},
$$

we have

$$
\mathbb{E}_{N} f(x)=\sum_{\mu \in \mathbb{Z}^{d}} \mathbb{1}_{I_{N, \mu}}(x) 2^{N d} \int_{I_{N, \mu}} f(y) d y,
$$

at least for $f \in L_{l o c}^{1}\left(\mathbb{R}^{d}\right)$.

The relation with the Haar system is given via the martingale difference operator $\mathbb{E}_{N+1}-\mathbb{E}_{N}$ which is the orthogonal projection onto the space generated by the Haar functions with frequency $2^{N}$, i.e.

$$
\mathbb{E}_{N+1} f-\mathbb{E}_{N} f=\sum_{\epsilon \in \Upsilon} \sum_{\mu \in \mathbb{Z}^{d}} 2^{N d}\left\langle f, h_{N, \mu}^{\epsilon}\right\rangle h_{N, \mu}^{\epsilon} .
$$

In addition to $\mathbb{E}_{N}$ we shall need another operator which involves Haar functions of a fixed frequency level. For $N \in \mathbb{N}$ and any $\mathfrak{a} \in \ell^{\infty}\left(\mathbb{Z}^{d} \times \Upsilon\right)$ we 
set

$$
T_{N}[f, \mathfrak{a}]=\sum_{\epsilon \in \Upsilon} \sum_{\mu \in \mathbb{Z}^{d}} a_{\mu, \epsilon} 2^{N d}\left\langle f, h_{N, \mu}^{\epsilon}\right\rangle h_{N, \mu}^{\epsilon}
$$

One aims for estimates of the operators $f \mapsto T_{N}[f, \mathfrak{a}]$ that are uniform in $\|\mathfrak{a}\|_{\infty} \leq 1$. The relation between the partial sum operators $S_{R}^{\mathcal{U}}$ and the operators $\mathbb{E}_{N}$ and $T_{N}[\cdot, \mathfrak{a}]$ is explained in $\oint 9$. In particular, the uniform boundedness of these operators in $B_{p, q}^{s}$ implies the local basic sequence property for all strongly admissible enumerations $\mathcal{U}$. The region of uniform boundedness for these operators is given in the next theorems, and depicted in Figure 3 .

Theorem 1.8. Let $0<p<\infty, 0<q \leq \infty$ and $s \in \mathbb{R}$.

(a) The operators $\mathbb{E}_{N}$ admit an extension from $\mathcal{S}\left(\mathbb{R}^{d}\right)$ to $B_{p, q}^{s}\left(\mathbb{R}^{d}\right)$ such that

$$
\sup _{N \geq 0}\left\|\mathbb{E}_{N}\right\|_{B_{p, q}^{s} \rightarrow B_{p, q}^{s}}<\infty
$$

if and only if one of the following six conditions is satisfied:

(i) $\quad p>1, \quad s=\frac{1}{p}, \quad q=\infty$,

(ii) $\quad p \geq 1, \quad-1+\frac{1}{p}<s<\frac{1}{p}, \quad 0<q \leq \infty$,

(iii) $\quad p \geq 1, \quad s=-1+\frac{1}{p}, \quad 0<q \leq 1$,

(iv) $\quad \frac{d}{d+1}<p<1, \quad s=1, \quad 0<q \leq p$,

(v) $\frac{d}{d+1}<p<1, \quad d\left(\frac{1}{p}-1\right)<s<1, \quad 0<q \leq \infty$,

(vi) $\quad \frac{d}{d+1} \leq p<1, \quad s=d\left(\frac{1}{p}-1\right), \quad 0<q \leq p$.

(b) If one of the conditions (i)-(vi) is satisfied and if $\|\mathfrak{a}\|_{\ell^{\infty}\left(\mathbb{Z}^{d} \times \Upsilon\right)} \leq 1$ then the operators $T_{N}[\cdot, \mathfrak{a}]$ are uniformly bounded on $B_{p, q}^{s}\left(\mathbb{R}^{d}\right)$.

Finally we state a result for $p=\infty$.

Theorem 1.9. (i) If $-1<s<0$ then the operators $\mathbb{E}_{N}$ have uniformly bounded extensions to $B_{\infty, q}^{s}\left(\mathbb{R}^{d}\right)$, for all $0<q \leq \infty$.

(ii) If $s=0$ then $\mathbb{E}_{N}$ admits a bounded extension to $B_{\infty, q}^{0}\left(\mathbb{R}^{d}\right)$ if and only if $q=\infty$. Moreover, we have $\sup _{N}\left\|\mathbb{E}_{N}\right\|_{B_{\infty, \infty}^{0} \rightarrow B_{\infty, \infty}^{0}}<\infty$.

(iii) If $s=-1$ then $\sup _{N}\left\|\mathbb{E}_{N}\right\|_{B_{\infty, q}^{-1} \rightarrow B_{\infty, q}^{-1}}=\infty$, for all $0<q \leq \infty$.

Moreover, below we also investigate situations when the individual operators $\mathbb{E}_{N}$ are bounded but not uniformly bounded, and derive precise growth conditions for the operator norms in such cases. See Theorem 6.1 for complete results in the case $s=1$, and Theorem 10.2 for the case $s=d / p-d$ and $p \leq 1$. A more detailed description of these and other local results is given the next subsection. 


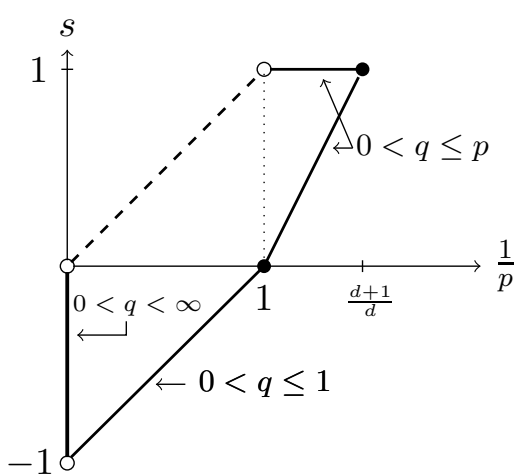

The cases $0<q<\infty$

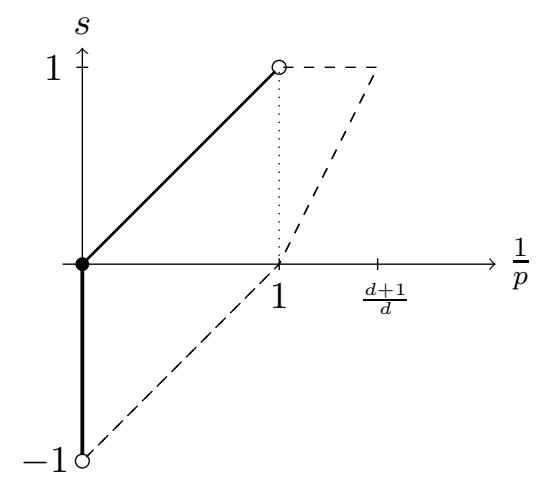

The case $q=\infty$

FiguRE 3. Regions for uniform boundedness of $\mathbb{E}_{N}$ (hence for the local basic sequence property) in the spaces $B_{p, q}^{s}\left(\mathbb{R}^{d}\right)$.

Guide through this paper and discussion of further quantitative results. The positive results in the interior of the pentagon $\mathfrak{P}$ in Figure 1 , including the unconditionality property, are classical and due to Triebel [13, 14, 19]. Moreover, unboundedness results outside the closure of $\mathfrak{P}$ are discussed in those references and [4].

The new positive results in Theorems 1.8 and 1.9 at the boundary of $\mathfrak{P}$ rely on $L^{p}$ bounds for the operators $L_{k} \mathbb{E}_{N} L_{j}$, where $L_{k}$ are suitable local means and the operators act on functions with compactly supported Fourier transforms. These bounds were already contained in our previous paper [4] (see also [5] for a proof of such results using wavelets). We review these estimates in 92 , see in particular Corollary 2.3. For both ranges $p \geq 1$ and $p \leq 1$ further straightforward estimates imply four key propositions with different outcomes in the four cases depending on the signs of $j-N$ and $k-N$. These propositions are stated for $p \leq 1$ in $\$ 3$ and for $p \geq 1$ in 8 .

Concerning the negative results in Theorems 1.8 and 1.9, these are presented as follows. First, when $s=1 / p$, characteristic functions of cubes (and also Haar functions) do not belong to $B_{p, q}^{1 / p}$ when $q<\infty$ which rules out these cases. In $\$ 5$ we shall further show that the space $b_{p, \infty}^{1 / p}$ (the closure of the Schwartz class under the $B_{p, \infty}^{1 / p}$ norm) intersects the algebraic span of $\mathcal{H}_{d}$ only in $\{0\}$. This is in contrast with the fact, shown in $8 \underline{8.2}$, that $b_{p, \infty}^{1 / p}$ is actually contained in ${\overline{\operatorname{span}} \mathcal{H}_{d}}^{B_{p, \infty}^{1 / p}}$ if $1<p<\infty$, so some positive result will hold in this case; see Proposition 9.2.

In $\sqrt[6]{6}$ we consider the cases $s=1$. At the endpoint space $B_{1, \infty}^{1}$ we show that the operators $\mathbb{E}_{N}$ are individually bounded, but not uniformly bounded, and for large $N$ we have $\left\|\mathbb{E}_{N}\right\|_{B_{1, \infty}^{1} \rightarrow B_{1, \infty}^{1}} \approx N$, see Theorem 6.1. 
When $s=1$ and $\frac{d}{d+1}<p<1$ the operators $\mathbb{E}_{N}$ are also individually bounded on $B_{p, q}^{1}$ but not uniformly bounded if $q>p$. In these cases Theorem 6.1 implies that for large $N$ we have $\left\|\mathbb{E}_{N}\right\|_{B_{p, q}^{1} \rightarrow B_{p, q}^{1}} \approx N^{1 / p-1 / q}$. The situation is worse at the endpoint $p=d /(d+1)$, that is the vertex of $\mathfrak{P}$ where $s=1=d / p-d$. In this case Theorem 10.2 gives an exponential lower bound even for compactly supported functions, while the $\mathbb{E}_{N}$ fail to be individually bounded in the whole $B_{d /(d+1), q}^{1}\left(\mathbb{R}^{d}\right)$ when $q>p=\frac{d}{d+1}$.

In 97 we discuss the simpler situation on the line $s=1 / p-1$ with $1<$ $p \leq \infty$. The cases $q>1$ are easily ruled out because Haar functions do not belong to the dual space $\left(B_{p, q}^{1 / p-1}\right)^{*}=B_{p^{\prime}, q^{\prime}}^{1 / p^{\prime}}$. In the cases $0<q \leq 1$, a lower bound $\left\|\mathbb{E}_{N}\right\|_{B_{\infty, q}^{-1} \rightarrow B_{\infty, q}^{-1}} \gtrsim N$ is obtained by duality in $\$ 7.2$.

In $\$ 10$ we gather the negative results for Theorem 1.8 at the edge $s=d / p-$ $d$ with $p \leq 1$. Again, we rule out the cases $q>1$, as Haar functions do not belong to the dual space $\left(B_{p, q}^{d / p-d}\right)^{*}=B_{\infty, q^{\prime}}^{0}$. Moreover, we show in Theorem 10.1 that the individual operators $\mathbb{E}_{N}$ are unbounded on $B_{p, q}^{d / p-d}$ when $q>p$. We shall actually prove sharp results if one restricts to compactly supported functions. To quantify these we use the following definition.

Definition 1.10. Let $Q$ be an open dyadic cube in $\mathbb{R}^{d}$ of side length $\geq 1 / 2$ and $X$ be a (quasi-)Banach space of tempered distributions $\mathcal{S}^{\prime}\left(\mathbb{R}^{d}\right)$. For a linear operator $T$ defined on those $f \in X$ which are supported in $Q$ we set

$$
\mathrm{Op}(T, X, Q)=\sup \left\{\|T f\|_{X}:\|f\|_{X} \leq 1, \operatorname{supp}(f) \subset Q\right\} .
$$

In Theorem 10.2 the precise growth of $\mathrm{Op}\left(\mathbb{E}_{N}, B_{p, q}^{d / p-d}, Q\right)$ is obtained for the range $\frac{d}{d+1} \leq p \leq q \leq 1$ where it is shown that

$$
\mathrm{Op}\left(\mathbb{E}_{N}, B_{p, q}^{d / p-d}, Q\right) \approx\left(2^{N d}|Q|\right)^{\frac{1}{p}-\frac{1}{q}}
$$

We also show that the above-mentioned lower bounds for $s=1$ have a sharp local analogue, namely for every cube $Q$ of sidelength $\geq 1$ one has

$$
\mathrm{Op}\left(\mathbb{E}_{N}, B_{p, q}^{1}, Q\right) \approx N^{\frac{1}{p}-\frac{1}{q}}, \quad \frac{d}{d+1}<p<1, \quad q \in[p, \infty] .
$$

We now turn to the uniform boundedness of the operators $S_{R}^{\mathcal{U}}$, associated with strongly admissible enumerations $\mathcal{U}$. We prove in 99.1 that if $\mathbb{E}_{N}$ (and $\left.T_{N}[\cdot, \mathfrak{a}]\right)$ are uniformly bounded in $B_{p, q}^{s}$ then we also have, for each fixed $Q$,

$$
\sup _{R \in \mathbb{N}} \operatorname{Op}\left(S_{R}^{\mathcal{U}}, B_{p, q}^{s}, Q\right)<\infty .
$$

Assuming (11), one has the local Schauder basis property if and only if the span of the Haar system is dense in $B_{p, q}^{s}$.

The density of span $\mathcal{H}_{d}$ in $B_{p, q}^{s}$ is studied separately in 88 , It clearly fails when $p=\infty$ or $q=\infty$ because $B_{p, q}^{s}$ is not separable in those cases. When $s=1$, we also show that density fails in $B_{p, q}^{1}$ when $\frac{d}{d+1} \leq p<1$ and $q \leq p$. This gives the negative results in Theorem 1.4 for those cases. We do not 
know, however, whether density should also fail in the remaining cases $q>p$; see our discussion in 98.1 .

The positive Schauder basis results in Theorem 1.3 are obtained in 89.2 , They follow from $\oint 9.1$ and the fact that the $B_{p, q}^{s}\left(\mathbb{R}^{d}\right)$-norms can be 'localized' if and only if $p=q$. Moreover, we actually prove the Schauder basis (resp. basic sequence) property in the Bourdaud spaces $\left(B_{p, q}^{s}\right)_{\ell p}$, in the range of Theorem 1.4 (resp. 1.8 and 1.9). We remark that these spaces coincide with $B_{p, q}^{s}$ if and only if $p=q$. Alternatively, the positive statement in (iii) of Theorem 1.3 is also a special case of a more general result for TriebelLizorkin spaces $F_{p, q}^{s}\left(\mathbb{R}^{d}\right)$ in $[\underline{6}$.

In $\$ 11$ we construct an explicit strongly admissible enumeration $\mathcal{U}$ for which

$$
S_{R(m)}^{\mathcal{U}} f=\mathbb{E}_{m} f \quad \text { if } \operatorname{supp}(f) \subset(-5,5)^{d},
$$

for a suitable sequence $R(m)$. One can then apply the examples on unboundedness of $\mathbb{E}_{N}$ when restricted to functions on cubes, alluded to above, to see that (11) fails. This gives the negative results in Theorems 1.4 and 1.3 at the edge $s=d / p-d$, for $\frac{d}{d+1}<p \leq 1$ and all $q>p$.

This same enumeration $\mathcal{U}$ is used in $\$ 12$ to show that, when $q \in(0, p)$, the operators $S_{R}^{\mathcal{U}}$ are not uniformly bounded in the whole spaces $B_{p, q}^{d / p-d}\left(\mathbb{R}^{d}\right)$ if $\frac{d}{d+1}<p \leq 1$, or $B_{p, q}^{1 / p-1}\left(\mathbb{R}^{d}\right)$ if $1<p<\infty$. Hence $\mathcal{U}$ is not a Schauder basis in these cases.

Finally, regarding the negative results in Theorem 1.1, examples showing the failure of unconditionality for parameters $(1 / p, s)$ on the boundary of $\mathfrak{P}$ are given in $\$ 13$ for the case $B_{p, q}^{d / p-d}$ with $\frac{d}{d+1}<p \leq 1$, and in $\$ 14$ for the case $B_{p, q}^{1 / p-1}$ with $1<p<\infty$. Since the argument in $₫ 13$ also applies to a similar result for Triebel-Lizorkin spaces, we include lower bounds for those as well.

Acknowledgements. The authors would like to thank the Isaac Newton Institute for Mathematical Sciences, Cambridge, for support and hospitality during the program Approximation, Sampling and Compression in Data Science where some work on this paper was undertaken. This work was supported by EPSRC grant no EP/K032208/1. G.G. was supported in part by grants MTM2016-76566-P, MTM2017-83262-C2-2-P and Programa Salvador de Madariaga PRX18/451 from Micinn (Spain), and grant 20906/PI/18 from Fundación Séneca (Región de Murcia, Spain). A.S. was supported in part by National Science Foundation grants DMS 1500162 and 1764295. T.U. was supported in part by Deutsche Forschungsgemeinschaft (DFG), grant $403 / 2-1$. 


\section{Preparatory Results}

2.1. Besov quasi-norms. Let $s \in \mathbb{R}$ and $0<p \leq \infty$ be given. Throughout the paper we fix a number $A>d / p$ and an integer

$$
M>A+|s|+2 .
$$

Consider two functions $\beta_{0}, \beta \in C_{c}^{\infty}\left(\mathbb{R}^{d}\right)$, supported in $(-1 / 2,1 / 2)^{d}$, with the properties $\left|\widehat{\beta}_{0}(\xi)\right|>0$ if $|\xi| \leq 1,|\widehat{\beta}(\xi)|>0$ if $1 / 8 \leq|\xi| \leq 1$ and $\beta$ has vanishing moments up to order $M$, that is

$$
\int_{\mathbb{R}^{d}} \beta(x) x_{1}^{m_{1}} \cdots x_{d}^{m_{d}} d x=0, \quad \forall m_{i} \in \mathbb{N}_{0} \text { with } m_{1}+\ldots+m_{d} \leq M .
$$

The optimal value of $M$ is irrelevant for the purposes of this paper, and (12) suffices for our results. We let $\beta_{k}:=2^{k d} \beta\left(2^{k} \cdot\right)$ for each $k \geq 1$, and denote

$$
L_{k}(f)=\beta_{k} * f
$$

whenever $f \in \mathcal{S}^{\prime}\left(\mathbb{R}^{d}\right)$. It is then known, see e.g. [17, 2.5.3], that an equivalent quasi-norm in the Besov spaces $B_{p, q}^{s}\left(\mathbb{R}^{d}\right), 0<q \leq \infty$, is given by

$$
\|g\|_{B_{p, q}^{s}} \approx\left\|\left\{2^{k s} L_{k} g\right\}_{k=0}^{\infty}\right\|_{\ell^{q}\left(L^{p}\right)} .
$$

Recall also that $b_{p, q}^{s}$ denotes the closure of $\mathcal{S}\left(\mathbb{R}^{d}\right)$ in the $B_{p, q}^{s}$ norm. When $p<\infty$ and $q=\infty$, it not difficult to see that $b_{p, \infty}^{s}$ coincides with the set of all $g \in \mathcal{S}^{\prime}\left(\mathbb{R}^{d}\right)$ such that

$$
\lim _{k \rightarrow \infty} 2^{k s}\left\|L_{k} g\right\|_{p}=0
$$

The space $b_{\infty, \infty}^{s}$ coincides with the set of all $g \in \mathcal{S}^{\prime}\left(\mathbb{R}^{d}\right)$ such that $L_{k} g \in C_{0}$ for all $k \in \mathbb{N}$ and such that $\lim _{k \rightarrow \infty} 2^{k s}\left\|L_{k} g\right\|_{\infty}=0$.

Next, let $\eta_{0} \in C_{c}^{\infty}\left(\mathbb{R}^{d}\right)$ be supported on $\{\xi:|\xi|<3 / 8\}$ and such that $\eta_{0}(\xi)=1$ if $|\xi| \leq 1 / 4$. We consider the following frequency localization operators

$$
\begin{aligned}
\widehat{\Lambda_{0} f}(\xi) & =\frac{\eta_{0}(\xi)}{\widehat{\beta}_{0}(\xi)} \widehat{f}(\xi), \\
\widehat{\Lambda_{k} f}(\xi) & =\frac{\eta_{0}\left(2^{-k} \xi\right)-\eta_{0}\left(2^{-k+1} \xi\right)}{\widehat{\beta}\left(2^{-k} \xi\right)} \widehat{f}(\xi), \quad k \geq 1,
\end{aligned}
$$

so that $f=\sum_{j=0}^{\infty} L_{j} \Lambda_{j} f$ with convergence in $\mathcal{S}^{\prime}$. It is also well-known that

$$
\|f\|_{B_{p, q}^{s}} \approx\left\|\left\{2^{k s} \Lambda_{k} f\right\}_{k=0}^{\infty}\right\|_{\ell^{q}\left(L^{p}\right)} .
$$

In particular, if we let $\Pi_{N}=\sum_{j=0}^{N} L_{j} \Lambda_{j}$, then

$$
\sup _{N}\left\|\Pi_{N} f\right\|_{B_{p, q}^{s}} \lesssim\|f\|_{B_{p, q}^{s}} \text {. }
$$


Below we shall be interested in uniformly bounded extensions of the dyadic averaging operators $\mathbb{E}_{N}$ defined in (7). Observe that

$$
\mathbb{E}_{N}-\Pi_{N}=\mathbb{E}_{N}\left(I-\Pi_{N}\right)-\left(I-\mathbb{E}_{N}\right) \Pi_{N},
$$

so if we denote

$$
\mathbb{E}_{N}^{\perp}=I-\mathbb{E}_{N}
$$

then, using (14), we have

$$
\begin{aligned}
\left\|\mathbb{E}_{N} f-\Pi_{N} f\right\|_{B_{p, q}^{s}} \lesssim & \left\|\left\{2^{k s} \sum_{j=N+1}^{\infty} L_{k} \mathbb{E}_{N} L_{j} \Lambda_{j} f\right\}_{k=0}^{\infty}\right\|_{\ell q\left(L^{p}\right)}+ \\
& +\left\|\left\{2^{k s} \sum_{j=0}^{N} L_{k} \mathbb{E}_{N}^{\perp} L_{j} \Lambda_{j} f\right\}_{k=0}^{\infty}\right\|_{\ell^{q}\left(L^{p}\right)} .
\end{aligned}
$$

Thus, as in 4, the uniform bounds of $\mathbb{E}_{N}$ will be reduced to suitable estimates for the compositions $L_{k} \mathbb{E}_{N} L_{j}$ and $L_{k} \mathbb{E}_{N}^{\perp} L_{j}$, for each $j, k \geq 0$.

2.2. Local estimates. We consider the following Peetre maximal operators: if $j \geq 0$ and $g$ is continuous, then

$$
\begin{aligned}
\mathcal{M}_{j} g(x) & =\sup _{|h|_{\infty} \leq 2^{-j+5}}|g(x+h)|, \\
\mathcal{M}_{A, j}^{*} g(x) & =\sup _{|h|_{\infty} \leq 2^{5}} \frac{|g(x+h)|}{\left(1+2^{j}|h|\right)^{A}}, \\
\mathfrak{M}_{A, j}^{* *} g(x) & =\sup _{h \in \mathbb{R}^{d}} \frac{|g(x+h)|}{\left(1+2^{j}|h|\right)^{A}} .
\end{aligned}
$$

Clearly, we have the pointwise relations $\mathcal{M}_{j} g \lesssim \mathcal{M}_{A, j}^{*} g \leq \mathfrak{M}_{A, j}^{* *} g$.

The following lemma was proved in [4, Lemma 2.2] using the cancellation properties of $L_{\max \{j, k\}}$.

Lemma 2.1. For $j, k \geq 0$ we have

$$
\left|L_{k} L_{j} g(x)\right| \lesssim 2^{-|k-j|(M-A)} \mathcal{M}_{A, \max \{j, k\}}^{*} g(x) .
$$

We remark that the larger maximal function $\mathfrak{M}_{A, \max \{j, k\}}^{* *} f$ was used in [4. Lemma 2.2], in place of $\mathcal{M}_{A, \max \{j, k\}}^{*} f$. However, since the convolution kernel of $L_{j} L_{k}$ is supported on a cube of sidelength $2^{-j}+2^{-k}$, it is clear that (20) holds as well.

From our previous work [4] we have the following crucial estimates.

Proposition 2.2. Let $0<p \leq \infty$ and

$$
B_{p}(j, k, N)= \begin{cases}2^{N-j} 2^{\frac{j-k}{p}} 2^{(j-N)(d-1)\left(\frac{1}{p}-1\right)_{+}} & \text {if } j, k>N, \\ 2^{\frac{N-k}{p}} 2^{j-N} & \text { if } j \leq N<k, \\ 2^{k-N} 2^{j-N} 2^{(N-k) d\left(\frac{1}{p}-1\right)_{+}} & \text {if } 0 \leq j, k \leq N, \\ 2^{k-j+\frac{j-N}{p}+[N-k+(j-k)(d-1)]\left(\frac{1}{p}-1\right)_{+}} & \text {if } k \leq N<j .\end{cases}
$$


Then the following inequalities hold for all continuous functions $g$ :

(i) For $j \geq N+1$,

$$
\begin{aligned}
& \left\|L_{k} \mathbb{E}_{N}\left[L_{j} g\right]\right\|_{p} \\
& \quad \lesssim \begin{cases}B_{p}(j, k, N)\left\|\mathcal{M}_{j} g\right\|_{p} & \text { if } k \geq N+1, \\
B_{p}(j, k, N)\left\|\mathcal{M}_{j} g\right\|_{p}+2^{-|j-k|(M-A)}\left\|\mathcal{M}_{A, j}^{*} g\right\|_{p} & \text { if } 0 \leq k \leq N .\end{cases}
\end{aligned}
$$

(ii) For $0 \leq j \leq N$,

$$
\begin{aligned}
\left\|L_{k} \mathbb{E}_{N}^{\perp}\left[L_{j} g\right]\right\|_{p} \\
\quad \lesssim \begin{cases}B_{p}(j, k, N)\left\|\mathcal{M}_{j} g\right\|_{p}+2^{-|j-k|(M-A)}\left\|\mathcal{M}_{A, j}^{*} g\right\|_{p} & \text { if } k \geq N+1, \\
B_{p}(j, k, N)\left\|\mathcal{M}_{j} g\right\|_{p} & \text { if } 0 \leq k \leq N .\end{cases}
\end{aligned}
$$

(iii) The same bounds hold if the operators $\mathbb{E}_{N}$ in (i) and $\mathbb{E}_{N}^{\perp}$ in (ii) are replaced by $T_{N}[\cdot, \mathfrak{a}]$ (as defined in (9)), uniformly in $\|\mathfrak{a}\|_{\infty} \leq 1$.

Remark. These bounds are contained in [4, §2], although the statement of [4, Proposition 2.1] is slightly less general. Namely, applying these bounds to $g \in \mathcal{S}^{\prime}\left(\mathbb{R}^{d}\right)$ such that $\operatorname{supp} \widehat{g} \subset\left\{|\xi| \leq 2^{j+1}\right\}$, and using additionally the Peetre inequality $\left\|\mathfrak{M}_{A, j}^{* *} g\right\|_{p} \lesssim\|g\|_{p}$, for $A>d / p$, one obtains [4, Proposition 2.1]. The formulation here will be applied later to functions of the form $g=\zeta \Lambda_{j} f$ with $f \in \mathcal{S}^{\prime}\left(\mathbb{R}^{d}\right)$ and $\zeta \in C_{c}^{\infty}$.

The statement of Proposition 2.2 can be put into a more convenient form. First, when $g=\Lambda_{j} f$, the Peetre maximal inequality gives $\left\|\mathfrak{M}_{A, j}^{* *}\left(\Lambda_{j} f\right)\right\|_{p} \lesssim$ $\left\|\Lambda_{j} f\right\|_{p}$ provided that $A>d / p$. Next, if $M \geq A+1$ then in the cases $k \leq$ $N<j$ and $j \leq N<k$ the term $2^{-|j-k|(M-A)}$ is dominated by $B_{p}(j, k, N)$ and thus the statement can be simplified. Finally, we shall use the quantities

$$
U_{p, s}(j, k, N):=2^{(k-j) s} B_{p}(j, k, N) ;
$$

see also (27) and (33) below. We then obtain

Corollary 2.3. Let $U_{p, s}(j, k, N)$ be as in (24). Then for all $f \in \mathcal{S}^{\prime}\left(\mathbb{R}^{d}\right)$

$$
2^{k s}\left\|L_{k} \mathbb{E}_{N} L_{j} \Lambda_{j} f\right\|_{p} \lesssim U_{p, s}(j, k, N) 2^{j s}\left\|\Lambda_{j} f\right\|_{p}, \quad \text { if } j \geq N+1,
$$

and

$$
2^{k s}\left\|L_{k} \mathbb{E}_{N}^{\perp} L_{j} \Lambda_{j} f\right\|_{p} \lesssim U_{p, s}(j, k, N) 2^{j s}\left\|\Lambda_{j} f\right\|_{p}, \quad \text { if } j \leq N .
$$

The same holds with $\mathbb{E}_{N}$ and $\mathbb{E}_{N}^{\perp}$ replaced by $T_{N}[\cdot, \mathfrak{a}]$ if $\|\mathfrak{a}\|_{\infty} \leq 1$. 


\section{UPPER BOUNDS FOR $p \leq 1$}

In the range $p \leq 1$, the constants in (24) take the following explicit form

$$
U_{p, s}(j, k, N)= \begin{cases}2^{k\left(s-\frac{1}{p}\right)} 2^{j\left(\frac{d}{p}-d-s\right)} 2^{N\left(d-\frac{d-1}{p}\right)} & \text { if } j, k>N \\ 2^{k\left(s-\frac{1}{p}\right)} 2^{j(1-s)} 2^{N\left(\frac{1}{p}-1\right)} & \text { if } j \leq N<k \\ 2^{k\left(s+d+1-\frac{d}{p}\right)} 2^{j(1-s)} 2^{N\left(\frac{d}{p}-d-2\right)} & \text { if } 0 \leq j, k \leq N \\ 2^{k\left(s+d+1-\frac{d}{p}\right)} 2^{j\left(\frac{d}{p}-d-s\right)} 2^{-N} & \text { if } k \leq N<j .\end{cases}
$$

We now state four propositions corresponding to the four cases of (27). We then sketch the straightforward proofs.

Proposition 3.1. For $\frac{d-1}{d}<p \leq 1$ and $r>0$,

$$
\begin{aligned}
\left(\sum_{k>N} 2^{k s r}\left\|\sum_{j>N} L_{k} \mathbb{E}_{N} L_{j} \Lambda_{j} f\right\|_{p}^{r}\right)^{1 / r} & \\
& \lesssim \begin{cases}\sup _{j>N} 2^{j s}\left\|\Lambda_{j} f\right\|_{p} & \text { if } d\left(\frac{1}{p}-1\right)<s<\frac{1}{p}, \\
\left(\sum_{j>N} 2^{j s p}\left\|\Lambda_{j} f\right\|_{p}^{p}\right)^{1 / p} & \text { if } s=d\left(\frac{1}{p}-1\right)<\frac{1}{p} .\end{cases}
\end{aligned}
$$

For $p=1$ and $s=1$ we have

$$
\sup _{k>N} 2^{k}\left\|\sum_{j>N} L_{k} \mathbb{E}_{N} L_{j} \Lambda_{j} f\right\|_{1} \lesssim \sup _{j>N} 2^{j}\left\|\Lambda_{j} f\right\|_{1} .
$$

The same inequalities hold when $\mathbb{E}_{N}$ is replaced with $T_{N}[\cdot, \mathfrak{a}]$ if $\|\mathfrak{a}\|_{\infty} \leq 1$.

Proposition 3.2. For $0<p<1$ and $r>0$,

$$
\begin{aligned}
\left(\sum_{k>N} 2^{k s r}\left\|\sum_{j \leq N} L_{k} \mathbb{E}_{N}^{\perp} L_{j} \Lambda_{j} f\right\|_{p}^{r}\right)^{1 / r} & \\
& \lesssim \begin{cases}\sup _{j \leq N} 2^{j s}\left\|\Lambda_{j} f\right\|_{p} & \text { if } s<1, \\
\left(\sum_{j=0}^{N} 2^{j s p}\left\|\Lambda_{j} f\right\|_{p}^{p}\right)^{1 / p} & \text { if } s=1 \\
2^{(s-1) N} \sup _{j \leq N} 2^{j s}\left\|\Lambda_{j} f\right\|_{p} & \text { if } 1<s<1 / p .\end{cases}
\end{aligned}
$$

Inequality (29a) also holds for $p=1$ and $s<1$. When $p=s=1$ we have

$$
\sup _{k>N} 2^{k}\left\|\sum_{j \leq N} L_{k} \mathbb{E}_{N}^{\perp} L_{j} \Lambda_{j} f\right\|_{1} \lesssim \sum_{j=0}^{N} 2^{j}\left\|\Lambda_{j} f\right\|_{1}
$$

The same statements hold with $\mathbb{E}_{N}^{\perp}$ replaced by $T_{N}[\cdot, \mathfrak{a}]$ if $\|\mathfrak{a}\|_{\infty} \leq 1$. 
Proposition 3.3. For $\frac{d}{d+2}<p \leq 1$ and $r>0$,

$$
\begin{aligned}
\left(\sum_{k \leq N} 2^{k s r} \|\right. & \left.\sum_{j \leq N} L_{k} \mathbb{E}_{N}^{\perp} L_{j} \Lambda_{j} f \|_{p}^{r}\right)^{1 / r} \\
& \lesssim \begin{cases}\sup _{j \leq N} 2^{j s}\left\|\Lambda_{j} f\right\|_{p} & \text { if } \frac{d}{p}-d-1<s<1, \\
\left(\sum_{j=0}^{N} 2^{j s p}\left\|\Lambda_{j} f\right\|_{p}^{p}\right)^{1 / p} & \text { if } s=1 \\
2^{(s-1) N} \sup _{j \leq N} 2^{j s}\left\|\Lambda_{j} f\right\|_{p} & \text { if } 1<s<1 / p .\end{cases}
\end{aligned}
$$

The same inequality holds with $\mathbb{E}_{N}^{\perp}$ replaced by $T_{N}[\cdot, \mathfrak{a}]$ if $\|\mathfrak{a}\|_{\infty} \leq 1$.

Proposition 3.4. For $0<p \leq 1$ and $r>0$,

$$
\begin{aligned}
\left(\sum_{k \leq N} 2^{k s r}\left\|\sum_{j>N} L_{k} \mathbb{E}_{N} L_{j} \Lambda_{j} f\right\|_{p}^{r}\right)^{1 / r} & \\
& \lesssim \begin{cases}\sup _{j>N} 2^{j s}\left\|\Lambda_{j} f\right\|_{p} & \text { if } s>\frac{d}{p}-d, \\
\left(\sum_{j>N} 2^{j s p}\left\|\Lambda_{j} f\right\|_{p}^{p}\right)^{1 / p} & \text { if } s=\frac{d}{p}-d .\end{cases}
\end{aligned}
$$

The same inequality holds with $\mathbb{E}_{N}$ replaced by $T_{N}[\cdot, \mathfrak{a}]$ if $\|\mathfrak{a}\|_{\infty} \leq 1$.

Proofs. The proofs of the four propositions involve Corollary 2.3 and an application of the $p$-triangle inequality for $p \leq 1$.

Proof of Proposition 3.1. First observe that the range of $s$ in (28a) is nontrivial if and only if $p>d /(d-1)$. Let $\Sigma_{r}$ denote the left hand side of (28a). Then the $p$-triangle inequality and Corollary 2.3 give

$$
\begin{aligned}
\Sigma_{r} & \leq\left(\sum_{k>N} 2^{k s r}\left[\sum_{j>N}\left\|L_{k} \mathbb{E}_{N} L_{j} \Lambda_{j} f\right\|_{p}^{p}\right]^{\frac{r}{p}}\right)^{1 / r} \\
& \lesssim\left(\sum_{k>N}\left[\sum_{j>N} U_{p, s}(j, k, N)^{p} 2^{j s p}\left\|\Lambda_{j} f\right\|_{p}^{p}\right]^{\frac{r}{p}}\right)^{1 / r} .
\end{aligned}
$$

When $d\left(\frac{1}{p}-1\right)<s<\frac{1}{p}$ this implies

$$
\Sigma_{r} \lesssim\left(\sum_{k>N}\left[\sum_{j>N} 2^{k\left(s-\frac{1}{p}\right) p} 2^{j\left(\frac{d}{p}-d-s\right) p} 2^{N\left(d-\frac{d-1}{p}\right) p}\right]^{\frac{r}{p}}\right)^{1 / r} \sup _{\ell>N} 2^{\ell s}\left\|\Lambda_{\ell} f\right\|_{p},
$$

which gives the asserted expression because the series above is bounded (uniformly in $N$ ). At the endpoint $s=d\left(\frac{1}{p}-1\right)<\frac{1}{p}$ we have

$$
\Sigma_{r} \lesssim\left(\sum_{k>N} 2^{(k-N)\left(s-\frac{1}{p}\right) r}\right)^{1 / r}\left(\sum_{j>N} 2^{j s p}\left\|\Lambda_{j} f\right\|_{p}^{p}\right)^{\frac{1}{p}}
$$


which also leads to the asserted expression in (28a). Finally, if $s=p=1$, using that $U_{p, s}(j, k, N)=2^{N-j}$ we obtain

$$
\Sigma_{\infty} \lesssim \sum_{j>N} 2^{N-j} 2^{j}\left\|\Lambda_{j} f\right\|_{1} \leq \sup _{\ell>N} 2^{\ell}\left\|\Lambda_{\ell} f\right\|_{1} .
$$

Proof of Proposition 3.2. The left hand side of (29a) is controlled by

$$
\begin{aligned}
& \left(\sum_{k>N}\left[\sum_{j \leq N} U_{p, s}(j, k, N)^{p} 2^{j s p}\left\|\Lambda_{j} f\right\|_{p}^{p}\right]^{r / p}\right)^{1 / r} \\
& \lesssim\left(\sum_{k \geq N} 2^{k\left(s-\frac{1}{p}\right) r} 2^{N\left(\frac{1}{p}-1\right) r}\right)^{\frac{1}{r}}\left(\sum_{j \leq N} 2^{j(1-s) p}\left[2^{j s}\left\|\Lambda_{j} f\right\|_{p}\right]^{p}\right)^{\frac{1}{p}} .
\end{aligned}
$$

If $s<1 / p$ the first sum can be evaluated as $C_{2}(p, s, r) 2^{N(s-1)}$ and the above expression is dominated by a constant times

$$
\left(\sum_{j \leq N} 2^{(j-N)(1-s) p}\left[2^{j s}\left\|\Lambda_{j} f\right\|_{p}\right]^{p}\right)^{\frac{1}{p}} .
$$

(29a) follows immediately. The proof of (29b) is similar.

Proof of Proposition [3.3. The left hand side of (30) is controlled by

$$
\begin{aligned}
& \left(\sum_{k \leq N}\left[\sum_{j \leq N} U_{p, s}(j, k, N)^{p} 2^{j s p}\left\|\Lambda_{j} f\right\|_{p}^{p}\right]^{r / p}\right)^{1 / r} \\
& \lesssim\left(\sum_{k \leq N} 2^{k\left(s+d+1-\frac{d}{p}\right) r} 2^{N\left(\frac{d}{p}-d-2\right) r}\right)^{\frac{1}{r}}\left(\sum_{j \leq N} 2^{j(1-s) p}\left[2^{j s}\left\|\Lambda_{j} f\right\|_{p}\right]^{p}\right)^{\frac{1}{p}} .
\end{aligned}
$$

If $s>\frac{d}{p}-d-1$ the first factor can be evaluated to be $C_{3}(p, s, r) 2^{N(s-1)}$ and the above expression is again dominated by a constant times

$$
\left(\sum_{j \leq N} 2^{(j-N)(1-s) p}\left[2^{j s}\left\|\Lambda_{j} f\right\|_{p}\right]^{p}\right)^{\frac{1}{p}} .
$$

Note that for the $s$-range in (30) to be nontrivial we want $\frac{d}{p}-d-1<1$, i.e. $p>\frac{d}{d+2}$. Now (30) follows easily.

Proof of Proposition 3.4. The left hand side of (31) is controlled by

$$
\begin{aligned}
& \left(\sum_{k \leq N}\left[\sum_{j>N} U_{p, s}(j, k, N)^{p} 2^{j s p}\left\|\Lambda_{j} f\right\|_{p}^{p}\right]^{r / p}\right)^{1 / r} \\
& \lesssim\left(\sum_{k \leq N} 2^{k\left(s+d+1-\frac{d}{p}\right) r} 2^{-N r}\right)^{\frac{1}{r}}\left(\sum_{j>N} 2^{j\left(\frac{d}{p}-d-s\right) p}\left[2^{j s}\left\|\Lambda_{j} f\right\|_{p}\right]^{p}\right)^{\frac{1}{p}} .
\end{aligned}
$$

In the range $s \geq \frac{d}{p}-d$ under consideration the first factor can be evaluated to be $C_{4}(p, s, r) 2^{N\left(s+d-\frac{d}{p}\right)}$ and the above expression is dominated by a constant 
times

$$
\left(\sum_{j>N} 2^{-(j-N)\left(s-\frac{d}{p}+d\right) p}\left[2^{j s}\left\|\Lambda_{j} f\right\|_{p}\right]^{p}\right)^{\frac{1}{p}} .
$$

This yields (31).

Remark 3.5. The proofs of Propositions 3.1 and 3.4 show that each operator $\mathbb{E}_{N}$ admits an extension to $B_{p, q}^{s}\left(\mathbb{R}^{d}\right)$ in the ranges of indices (iv), (v) and (vi) of Theorem 1.8, namely

$$
\mathbb{E}_{N}(f):=\sum_{j=0}^{\infty} \mathbb{E}_{N}\left[L_{j} \Lambda_{j} f\right], \quad \text { in } B_{p, q}^{s} .
$$

Indeed, for all $r>0$ and for $J_{2}>J_{1}>N$ one has, in cases (iv) and (v),

$$
\left\|\mathbb{E}_{N}\left(\sum_{j=J_{1}}^{J_{2}} L_{j} \Lambda_{j} f\right)\right\|_{B_{p, r}^{s}} \lesssim_{N} 2^{-J_{1} \varepsilon}\|f\|_{B_{p, \infty}^{s}},
$$

with $\varepsilon=s-d(1 / p-1)>0$, and in case (vi)

$$
\left\|\mathbb{E}_{N}\left(\sum_{j=J_{1}}^{J_{2}} L_{j} \Lambda_{j} f\right)\right\|_{B_{p, r}^{s}} \lesssim_{N}\left(\sum_{j=J_{1}}^{J_{2}} 2^{j s p}\left\|\Lambda_{j} f\right\|_{p}^{p}\right)^{\frac{1}{p}} .
$$

Proof of Theorem 1.8: Sufficiency for $\frac{d}{d+1} \leq p \leq 1$. In view of (18), (19) and trivial embeddings of Besov spaces, the uniform boundedness of $\mathbb{E}_{N}$ follows immediately from the above four propositions.

4. UPPER BOUNDS FOR $1 \leq p \leq \infty$

When $p \geq 1$ the constants in (24) take the form

$$
U_{p, s}(j, k, N)= \begin{cases}2^{k\left(s-\frac{1}{p}\right)} 2^{j\left(\frac{1}{p}-1-s\right)} 2^{N} & \text { if } j, k>N, \\ 2^{k\left(s-\frac{1}{p}\right)} 2^{j(1-s)} 2^{N\left(\frac{1}{p}-1\right)} & \text { if } j \leq N<k \\ 2^{k(1+s)} 2^{j(1-s)} 2^{-2 N} & \text { if } 0 \leq j, k \leq N, \\ 2^{k(1+s)} 2^{j\left(\frac{1}{p}-1-s\right)} 2^{-\frac{N}{p}} & \text { if } k \leq N<j .\end{cases}
$$

Again we state four propositions corresponding to the four cases of (33).

Proposition 4.1. Suppose $1 \leq p \leq \infty$. Then

$$
\sup _{k>N} 2^{k s}\left\|\sum_{j>N} L_{k} \mathbb{E}_{N} L_{j} \Lambda_{j} f\right\|_{p} \lesssim \sup _{j>N} 2^{j s}\left\|\Lambda_{j} f\right\|_{p}, \quad \text { if } \frac{1}{p}-1<s \leq \frac{1}{p} .
$$

Moreover, for all $r>0$

$$
\begin{aligned}
\left(\sum_{k>N} 2^{k s r}\left\|\sum_{j>N} L_{k} \mathbb{E}_{N} L_{j} \Lambda_{j} f\right\|_{p}^{r}\right)^{1 / r} & \\
& \lesssim \begin{cases}\sup _{j>N} 2^{j s}\left\|\Lambda_{j} f\right\|_{p} & \text { if } \frac{1}{p}-1<s<\frac{1}{p}, \\
\sum_{j>N} 2^{j s}\left\|\Lambda_{j} f\right\|_{p} & \text { if } s=\frac{1}{p}-1 .\end{cases}
\end{aligned}
$$


The same inequalities hold with $\mathbb{E}_{N}$ replaced by $T_{N}[\cdot, \mathfrak{a}]$ if $\|\mathfrak{a}\|_{\infty} \leq 1$.

Proposition 4.2. Suppose $1 \leq p \leq \infty$. Then for all $r>0$

$$
\left(\sum_{k>N} 2^{k s r}\left\|\sum_{j \leq N} L_{k} \mathbb{E}_{N}^{\perp} L_{j} \Lambda_{j} f\right\|_{p}^{r}\right)^{1 / r} \lesssim \sup _{j \leq N} 2^{j s}\left\|\Lambda_{j} f\right\|_{p}, \quad \text { if } s<\frac{1}{p} .
$$

Moreover, if $s=\frac{1}{p}<1$ then

$$
\sup _{k>N} 2^{k s}\left\|\sum_{j \leq N} L_{k} \mathbb{E}_{N}^{\perp} L_{j} \Lambda_{j} f\right\|_{p} \lesssim \sup _{j \leq N} 2^{j s}\left\|\Lambda_{j} f\right\|_{p}
$$

and if $s=p=1$ then

$$
\sup _{k>N} 2^{k}\left\|\sum_{j \leq N} L_{k} \mathbb{E}_{N}^{\perp} L_{j} \Lambda_{j} f\right\|_{1} \lesssim \sum_{j \leq N} 2^{j}\left\|\Lambda_{j} f\right\|_{1} .
$$

The same inequalities hold with $\mathbb{E}_{N}^{\perp}$ replaced by $T_{N}[\cdot, \mathfrak{a}]$ if $\|\mathfrak{a}\|_{\infty} \leq 1$.

Proposition 4.3. Let $1 \leq p \leq \infty$ and $r>0$. Then

$$
\left(\sum_{k \leq N} 2^{k s r}\left\|\sum_{j \leq N} L_{k} \mathbb{E}_{N}^{\perp} L_{j} \Lambda_{j} f\right\|_{p}^{r}\right)^{1 / r} \lesssim \sup _{j \leq N} 2^{j s}\left\|\Lambda_{j} f\right\|_{p} \text { if }-1<s<1 .
$$

Moreover, for the case $s=-1$ we have

$$
\sup _{k \leq N} 2^{-k}\left\|\sum_{j \leq N} L_{k} \mathbb{E}_{N}^{\perp} L_{j} \Lambda_{j} f\right\|_{p} \lesssim \sup _{j \leq N} 2^{-j}\left\|\Lambda_{j} f\right\|_{p},
$$

and for the case $s=1$ we have

$$
\left(\sum_{k \leq N} 2^{k r}\left\|\sum_{j \leq N} L_{k} \mathbb{E}_{N}^{\perp} L_{j} \Lambda_{j} f\right\|_{p}^{r}\right)^{1 / r} \lesssim \sum_{j=0}^{N} 2^{j}\left\|\Lambda_{j} f\right\|_{p}
$$

The same inequalities hold with $\mathbb{E}_{N}^{\perp}$ replaced by $T_{N}[\cdot, \mathfrak{a}]$ if $\|\mathfrak{a}\|_{\infty} \leq 1$.

Proposition 4.4. Let $1 \leq p \leq \infty$. Then for all $r>0$,

$$
\left(\sum_{k \leq N} 2^{k s r}\left\|\sum_{j>N} L_{k} \mathbb{E}_{N} L_{j} \Lambda_{j} f\right\|_{p}^{r}\right)^{1 / r} \lesssim \sup _{j>N} 2^{j s}\left\|\Lambda_{j} f\right\|_{p} \quad \text { if } s>\frac{1}{p}-1
$$

Moreover, for the case $s=\frac{1}{p}-1$ and $1 \leq p<\infty$,

$$
\left(\sum_{k \leq N} 2^{k\left(\frac{1}{p}-1\right) r}\left\|\sum_{j>N} L_{k} \mathbb{E}_{N} L_{j} \Lambda_{j} f\right\|_{p}^{r}\right)^{1 / r} \lesssim \sum_{j=N+1}^{\infty} 2^{j\left(\frac{1}{p}-1\right)}\left\|\Lambda_{j} f\right\|_{p} .
$$

Finally, for the case $s=-1$ and $p=\infty$

$$
\sup _{k \leq N} 2^{-k}\left\|\sum_{j>N} L_{k} \mathbb{E}_{N} L_{j} \Lambda_{j} f\right\|_{\infty} \lesssim \sum_{j=N+1}^{\infty} 2^{-j}\left\|\Lambda_{j} f\right\|_{\infty} .
$$

The same inequalities hold when $\mathbb{E}_{N}$ is replaced by $T_{N}[\cdot, \mathfrak{a}]$, with $\|\mathfrak{a}\|_{\infty} \leq 1$. 
Proofs. The proofs of the four propositions involve Corollary 2.3 and an application of the triangle inequality for $L^{p}$ when $p \geq 1$.

Proof of Proposition 4.1. Assume $s<1 / p$. By the triangle inequality and Corollary 2.3 the left hand side of (34b) is estimated by

$$
\begin{aligned}
& \left(\sum_{k>N} 2^{k s r}\left[\sum_{j>N}\left\|L_{k} \mathbb{E}_{N} L_{j} \Lambda_{j} f\right\|_{p}\right]^{r}\right)^{\frac{1}{r}} \\
& \lesssim\left(\sum_{k>N}\left[\sum_{j>N} U_{p, s}(j, k, N) 2^{j s}\left\|\Lambda_{j} f\right\|_{p}\right]^{r}\right)^{\frac{1}{r}} \\
& \lesssim\left(\sum_{k>N} 2^{k\left(s-\frac{1}{p}\right) r}\right)^{\frac{1}{r}} \sum_{j>N} 2^{j\left(\frac{1}{p}-1-s\right)} 2^{N} 2^{j s}\left\|\Lambda_{j} f\right\|_{p} .
\end{aligned}
$$

When $s<1 / p$ the first factor is $c(p, s, r) 2^{N(s-1 / p)}$ and we see that the entire expression is dominated by a constant times

$$
\sum_{j>N} 2^{(N-j)\left(s+1-\frac{1}{p}\right)} 2^{j s}\left\|\Lambda_{j} f\right\|_{p}
$$

which proves (34b) and of course also (34a) when $s<1 / p$. Replacing the $\ell^{r}$ norm by a supremum in the above proof we see that (34a) is valid even for $s=1 / p$.

Proof of Proposition 4.2. Let $s<1 / p$. The left hand side of (35a) is estimated by a constant times

$$
\begin{aligned}
& \left(\sum_{k>N}\left[\sum_{j \leq N} U_{p, s}(j, k, N) 2^{j s}\left\|\Lambda_{j} f\right\|_{p}\right]^{r}\right)^{\frac{1}{r}} \\
& \lesssim\left(\sum_{k>N} 2^{k\left(s-\frac{1}{p}\right) r}\right)^{\frac{1}{r}} \sum_{j \leq N} 2^{j(1-s)} 2^{N\left(\frac{1}{p}-1\right)} 2^{j s}\left\|\Lambda_{j} f\right\|_{p} \\
& \lesssim \sum_{j \leq N} 2^{(j-N)(1-s)} 2^{j s}\left\|\Lambda_{j} f\right\|_{p} .
\end{aligned}
$$

This easily yields (35a). The proofs of (35b), (35c) are similar.

Proof of Proposition 4.3. Assume $s>-1$. The left hand side of (36a) is estimated by a constant times

$$
\begin{aligned}
& \left(\sum_{k \leq N}\left[\sum_{j \leq N} U_{p, s}(j, k, N) 2^{j s}\left\|\Lambda_{j} f\right\|_{p}\right]^{r}\right)^{\frac{1}{r}} \\
& \lesssim\left(\sum_{k \leq N} 2^{k(1+s) r}\right)^{\frac{1}{r}} \sum_{j \leq N} 2^{j(1-s)} 2^{-2 N} 2^{j s}\left\|\Lambda_{j} f\right\|_{p},
\end{aligned}
$$


and since the first factor is $\tilde{c}(p, q, r) 2^{N(1+s)}$ we estimate the expression by a constant times

$$
\sum_{j \leq N} 2^{(j-N)(1-s)} 2^{j s}\left\|\Lambda_{j} f\right\|_{p}
$$

This easily yields (36a) and also (36c). The proof of (36b) which has a supremum in $k$ for the case $s=-1$ is similar.

Proof of Proposition 4.4. Let $s>-1$. The left hand side of (37a) is estimated by a constant times

$$
\begin{aligned}
& \left(\sum_{k \leq N}\left[\sum_{j>N} U_{p, s}(j, k, N) 2^{j s}\left\|\Lambda_{j} f\right\|_{p}\right]^{r}\right)^{\frac{1}{r}} \\
& \lesssim\left(\sum_{k \leq N} 2^{k(1+s) r}\right)^{\frac{1}{r}} \sum_{j>N} 2^{j\left(\frac{1}{p}-1-s\right)} 2^{-\frac{N}{p}} 2^{j s}\left\|\Lambda_{j} f\right\|_{p} \\
& \lesssim \sum_{j>N} 2^{(j-N)\left(\frac{1}{p}-1-s\right)} 2^{j s}\left\|\Lambda_{j} f\right\|_{p}
\end{aligned}
$$

which yields (37a ) and also (37b). The proof of (37c) for the case $s=-1$ is similar.

Remark 4.5. Similar reasonings as in Remark 3.5justify the meaning of the extension formula for $\mathbb{E}_{N}$ in (32), for the ranges of indices in (i), (ii), (iii) in Theorem 1.8, and the cases (i), (ii) in Theorem 1.9. In the special case $s=1 / p$, for $1<p \leq \infty$, one has

$$
\left\|\mathbb{E}_{N}\left(\sum_{j=J_{1}}^{J_{2}} L_{j} \Lambda_{j} f\right)\right\|_{B_{p, \infty}^{1 / p}} \lesssim_{N} 2^{-J_{1}}\|f\|_{B_{p, \infty}^{s}}
$$

so the series $\sum_{j=0}^{\infty} \mathbb{E}_{N}\left(L_{j} \Lambda_{j} f\right)$ always converges in $B_{p, \infty}^{1 / p}$, even though the series $\sum_{j=0}^{\infty} L_{j} \Lambda_{j} f$ only does if $f \in b_{p, \infty}^{1 / p}$.

Proof of Theorems 1.8 and 1.9: Sufficiency for $1 \leq p \leq \infty$. As before, one uses the previous four propositions combined with (18), (19) and trivial embeddings of Besov spaces.

\section{Necessary COnditions for boundedness When $s=1 / p$}

It is well known that the characteristic function of a cube (and also the Haar functions) do not belong to $B_{p, q}^{1 / p}$ for any $q<\infty$; see [15, 2.6.3 (18)]. In this section we elaborate a bit more on this result.

Recall that $b_{p, \infty}^{s}$ denotes the closure of the Schwartz space in the $B_{p, \infty}^{s}$ norm. Note also that $B_{p, q}^{s} \subset b_{p, \infty}^{s}$ for all $q<\infty$; see (15) above. Finally, $\operatorname{span} \mathcal{H}_{d}$ denotes the vector space of all finite linear combinations of Haar functions.

Proposition 5.1. Let $0<p \leq \infty$. Then

$$
b_{p, \infty}^{1 / p} \cap \operatorname{span} \mathcal{H}_{d}=\{0\} .
$$


Before proving this proposition we define, given $M \in \mathbb{N}$, certain test functions $\Psi$ with vanishing moments of order up to $2 M$ (which, along with their dilates $\Psi_{N}=2^{N d} \Psi\left(2^{N}\right.$.), will be also be used in subsequent sections).

5.1. Tensorized test functions. Given $M \in \mathbb{N}$, consider a non-negative even function $\phi_{0} \in C_{c}^{\infty}\left(-\frac{1}{8}, \frac{1}{8}\right)$ such that $\phi_{0}^{(2 M)}(t)>0$ for all $t$ in some interval $[-2 \varepsilon, 2 \varepsilon]$ (with $\varepsilon<1 / 16$ ). Since $\widehat{\phi}_{0}(0)=\int \phi_{0}>0$, dilating if necessary we may also assume that $\widehat{\phi}_{0} \neq 0$ on $(-1,1)$. Let $\varphi_{0} \in C_{c}^{\infty}\left(\left(-\frac{1}{8}, \frac{1}{8}\right)^{d-1}\right)$ be such that $\widehat{\varphi}_{0} \neq 0$ on $(-1,1)^{d-1}$ and $\widehat{\varphi}_{0}(0)=1$. For $M \geq 1$, let

$$
\theta(t)=\left(\frac{d}{d t}\right)^{2 M} \phi_{0}(t), \quad \vartheta\left(x_{2}, \ldots, x_{d}\right)=\left(\frac{\partial^{2}}{\partial x_{2}^{2}}+\cdots+\frac{\partial^{2}}{\partial x_{d}^{2}}\right)^{M} \varphi_{0}\left(x^{\prime}\right) .
$$

In one dimension the function $\vartheta$ is obsolete and we just define $\Psi=\theta$. If $d \geq 2$ we define

$$
\Psi(x)=\Delta^{M}\left[\phi_{0} \otimes \varphi_{0}\right](x)=\theta\left(x_{1}\right) \varphi_{0}\left(x^{\prime}\right)+\phi_{0}\left(x_{1}\right) \vartheta\left(x^{\prime}\right) .
$$

Clearly,

$$
\int_{\mathbb{R}^{d}} \Psi(y) y_{1}^{m_{1}} \cdots y_{d}^{m_{d}} d y=0, \quad \text { when } m_{1}+\ldots+m_{d}<2 M .
$$

If we choose $2 M \gg|s|+d / p-d$ then for all $f \in B_{p, q}^{s}\left(\mathbb{R}^{d}\right)$,

$$
\|f\|_{B_{p, q}^{s}} \gtrsim\left\|\left\{2^{k s} \Psi_{k} * f\right\}_{k \in \mathbb{N}}\right\|_{\ell^{q}\left(L^{p}\right)} .
$$

5.2. Proof of Proposition 5.1. We argue by contradiction and assume that there is a nontrivial $f \in b_{p, \infty}^{1 / p} \cap \operatorname{span} \mathcal{H}_{d}$. Then for some $N \in \mathbb{N}$ we can write $f$ as

$$
f=\sum_{\nu \in \Gamma} a_{\nu} \mathbb{1}_{I_{N, \nu}}
$$

where $I_{N, \nu}=\prod_{i=1}^{d}\left[\nu_{i} 2^{-N},\left(\nu_{i}+1\right) 2^{-N}\right), \Gamma$ is a finite nonempty subset of $\mathbb{Z}^{d}$, and $a_{\nu} \in \mathbb{C}$ with $a_{\nu} \neq 0$ for $\nu \in \Gamma$.

Consider the usual partial order in $\mathbb{Z}^{d}$, that is for $\mu, \nu \in \mathbb{Z}^{d}$ we say that

$$
\mu \leq \nu \quad \text { if } \quad \mu_{i} \leq \nu_{i} \quad \forall i=1, \ldots, d .
$$

Pick a minimal element $\mu \in \Gamma$, meaning that that if $\nu \in \Gamma$ and $\nu \leq \mu$ then necessarily $\nu=\mu$. Now consider the function

$$
g(x)=f\left(2^{-N}(x+\mu)\right) / a_{\mu},
$$

which also belongs to $b_{p, \infty}^{1 / p} \cap \operatorname{span} \mathcal{H}_{d}$. Note that $g$ is now a linear combination of disjoint unit cubes and satisfies

$$
g(x)= \begin{cases}1 & \text { if } \quad x \in[0,1)^{d} \\ 0 & \text { if } \quad x \in(-1,1)^{d} \backslash[0,1)^{d} .\end{cases}
$$

This last property is a consequence of the minimality of $\mu$. 
Consider now the function $\Psi \in C_{c}^{\infty}\left(\mathbb{R}^{d}\right)$ as in (38), with the pairs of functions $\phi_{0}, \theta$ and $\varphi_{0}, \vartheta$ as in the paragraph preceding that definition. So, in particular,

$$
\int_{\mathbb{R}^{d-1}} \varphi_{0}\left(x^{\prime}\right) d x^{\prime}=1 \quad \text { and } \quad \int_{\mathbb{R}^{d-1}} \vartheta\left(x^{\prime}\right) d x^{\prime}=0 .
$$

Observe further that for $t \in[-2 \varepsilon,-\varepsilon]$

$$
\int_{0}^{\infty} \theta(t-s) d s=\int_{-\infty}^{t} \theta(u) d u=-\int_{t}^{0} \theta(u) d u \leq-\int_{-\varepsilon}^{0} \theta(s) d s
$$

since $\int_{-\infty}^{0} \theta(s) d s=\phi_{0}^{(2 M-1)}(0)=0$ (because $\phi_{0}$ is even) and $\theta(u)>0$ for $u \in(-2 \varepsilon, 0)$. Thus, if we set

$$
c:=\int_{-\varepsilon}^{0} \theta(s) d s>0
$$

we obtain

$$
\int_{0}^{\infty} \theta(t-s) d s \leq-c, \quad \forall t \in[-2 \varepsilon,-\varepsilon] .
$$

Next consider $\Psi_{k}(x)=2^{k d} \Psi\left(2^{k} x\right), k \geq 1$, and note that $\Psi$ has enough vanishing moments so that

$$
\|h\|_{B_{p, \infty}^{1 / p}} \gtrsim \sup _{k \geq 1} 2^{k / p}\left\|\Psi_{k} * h\right\|_{p}, \quad h \in B_{p, \infty}^{1 / p} .
$$

Moreover, since $g \in b_{p, \infty}^{1 / p}$ we also have

$$
2^{k / p}\left\|\Psi_{k} * g\right\|_{p} \rightarrow 0, \quad \text { as } \quad k \rightarrow \infty .
$$

We show that this leads to a contradiction. Indeed, if $x_{1} \in\left[-\varepsilon 2^{1-k},-\varepsilon 2^{-k}\right]$ and $x^{\prime} \in[1 / 4,3 / 4]^{d-1}$ then, using that $\operatorname{supp} \Psi_{k}(x-\cdot) \subset(-1,1) \times(1 / 8,7 / 8)^{d-1}$, we may apply (40) and (41) to obtain

$$
\begin{aligned}
g * \Psi_{k}(x)= & \int_{[0,1)^{d}} \Psi_{k}(x-y) d y \\
= & \int_{0}^{1} \theta_{k}\left(x_{1}-y_{1}\right) d y_{1} \int_{(0,1)^{d-1}} \varphi_{0, k}\left(x^{\prime}-y^{\prime}\right) d y^{\prime}+ \\
& \quad+\int_{0}^{1} \phi_{0, k}\left(x_{1}-y_{1}\right) d y_{1} \int_{(0,1)^{d-1}} \vartheta_{k}\left(x^{\prime}-y^{\prime}\right) d y^{\prime} \\
= & 2^{k} \int_{0}^{1} \theta\left(2^{k}\left(x_{1}-y_{1}\right)\right) d y_{1}=\int_{0}^{2^{k}} \theta\left(2^{k} x_{1}-u\right) d u \\
= & \int_{0}^{\infty} \theta\left(2^{k} x_{1}-u\right) d u \leq-c / 2 .
\end{aligned}
$$

Thus we must have

$$
\left\|g * \Psi_{k}\right\|_{p} \geq(c / 2)\left(\varepsilon 2^{-k}\right)^{1 / p}(1 / 2)^{\frac{d-1}{p}},
$$

which contradicts (42). 
Remark. In the recent work [20] by Yuan, Sickel and Yang, the authors study regularity properties of the Haar system in other Besov-type spaces $B_{p, q}^{s, \tau}\left(\mathbb{R}^{d}\right)$ which serves as a first step to investigate its basis properties in these spaces.

\section{NeCESSARY CONDITIONS FOR BOUNDEDNESS WHEN $s=1$}

We now consider the necessity of the condition $q \leq p$ in part (iv) of Theorem 1.8. This restriction was also noticed in [8]. For $q>p$ we show that the operators $\mathbb{E}_{N}$ are bounded, but not uniformly bounded and determine the precise behavior of the operator norms as $N \rightarrow \infty$. The lower bounds will be obtained by testing with suitable functions with compact support; we refer to (10) for the notation in the next theorem.

Theorem 6.1. Suppose that either

(i) $\frac{d}{d+1}<p<1$ and $p \leq q \leq \infty$, or

(ii) $p=1$ and $q=\infty$.

Then for large $N$

$$
\left\|\mathbb{E}_{N}\right\|_{B_{p, q}^{1} \rightarrow B_{p, q}^{1}} \approx N^{\frac{1}{p}-\frac{1}{q}} .
$$

Moreover, for cubes $Q$ of side length $\geq 1 / 2$,

$$
\operatorname{Op}\left(\mathbb{E}_{N}, B_{p, q}^{1}, Q\right) \approx N^{\frac{1}{p}-\frac{1}{q}}
$$

Proof of the upper bounds in Theorem 6.1. Letting $s=1$ in Propositions 3.1 and 3.4 (and noticing that $d\left(\frac{1}{p}-1\right)<1<\frac{1}{p}$ when $\frac{d}{d+1}<p<1$ ), we see that

$$
\left\|\left\{2^{k} \sum_{j>N} L_{k} \mathbb{E}_{N} L_{j} \Lambda_{j} f\right\}_{k=0}^{\infty}\right\|_{\ell^{q}\left(L^{p}\right)} \lesssim\|f\|_{B_{p, \infty}^{1}} \leq\|f\|_{B_{p, q}^{1}} .
$$

On the other hand, letting $s=1$ in Propositions 3.2 and 3.3, and using Hölder's inequality we obtain

$$
\left\|\left\{2^{k} \sum_{j \leq N} L_{k} \mathbb{E}_{N}^{\perp} L_{j} \Lambda_{j} f\right\}_{k=0}^{\infty}\right\|_{\ell^{q}\left(L^{p}\right)} \lesssim\left(\sum_{j=0}^{N} 2^{j p}\left\|\Lambda_{j} f\right\|_{p}^{p}\right)^{1 / p} \lesssim N^{1 / p-1 / q}\|f\|_{B_{p, q}^{1}} .
$$

Combining this with (18) and (19) we obtain $\left\|\mathbb{E}_{N}\right\|_{B_{p, q}^{1} \rightarrow B_{p, q}^{1}} \lesssim N^{\frac{1}{p}-\frac{1}{q}}$. The above arguments also apply if $s=p=1$, provided we let $q=\infty$.

Proof of the lower bounds in Theorem 6.1. We shall actually prove a stronger result which gives a lower bound even for a $B_{p, q}^{1} \rightarrow B_{p, \infty}^{1}$ estimate and for functions supported in the open unit cube $Q_{0}=(0,1)^{d}$.

Theorem 6.2. If $0<p \leq 1$ and $p \leq q \leq \infty$, then there is $c_{p, q}>0$ such that, for each $N \geq 1$,

$$
\sup \left\{\left\|\mathbb{E}_{N} f\right\|_{B_{p, \infty}^{1}}:\|f\|_{B_{p, q}^{1}} \leq 1, \operatorname{supp}(f) \subset Q_{0}\right\} \geq c_{p, q} N^{\frac{1}{p}-\frac{1}{q}} .
$$


Fix $u \in C_{c}^{\infty}(\mathbb{R})$ supported in $(1 / 8,7 / 8)$ with $u(t)=1$ for $t \in[1 / 4,3 / 4]$, and $\chi \in C_{c}^{\infty}\left(\mathbb{R}^{d-1}\right)$ supported in $(1 / 8,7 / 8)^{d-1}$ with $\chi\left(x^{\prime}\right)=1$ for $x^{\prime} \in$ $(1 / 4,3 / 4)^{d-1}$; here $x^{\prime}=\left(x_{2}, \ldots, x_{d}\right)$. Define, for large $N$, functions of one variable

$$
g_{N, j}(t)=e^{2 \pi i 2^{j} t} u(N t-2 j), \quad j \in \mathbb{N}
$$

and let

$$
f_{N}(x)=\chi\left(x_{2}, \ldots, x_{d}\right) \sum_{N / 8<j<N / 4} 2^{-j} g_{N, j}\left(x_{1}\right) .
$$

Lemma 6.3. For $p \leq q \leq \infty$ we have

$$
\left\|f_{N}\right\|_{B_{p, q}^{1}} \lesssim N^{1 / q-1 / p}
$$

Proof. We estimate $L_{k} f_{N}=\beta_{k} * f_{N}$. If $2^{k} \leq N$, since $\beta_{k} * f_{N}$ is compactly supported and $\left\|\beta_{k} * f_{N}\right\|_{\infty} \lesssim\left\|f_{N}\right\|_{\infty} \lesssim 2^{-N / 8}$, then

$$
\left(\sum_{k=0}^{\log _{2} N} 2^{k q}\left\|\beta_{k} * f_{N}\right\|_{p}^{q}\right)^{1 / q} \lesssim N 2^{-N / 8} \ll N^{1 / q-1 / p} .
$$

Assume now that $2^{k}>N$. First notice that the sets

$$
\operatorname{supp} \beta_{k} * g_{N, j} \subset\left\{\frac{2 j}{N}+\left(-\frac{2}{N}, \frac{2}{N}\right)\right\} \times(0,1)^{d-1}, \quad \frac{N}{8}<j<\frac{N}{4},
$$

are pairwise disjoint, and thus

$$
\left\|\beta_{k} * f_{N}\right\|_{p}=\left(\sum_{\frac{N}{8}<j<\frac{N}{4}} 2^{-j p}\left\|\beta_{k} *\left(g_{N, j} \otimes \chi\right)\right\|_{p}^{p}\right)^{1 / p}
$$

Next, we distinguish the cases $j \geq k$ and $j \leq k$. When $j \geq k$, if we integrate by parts $M$-times with respect to $y_{1}$ in the convolution we obtain

$$
\beta_{k} *\left(g_{N, j} \otimes \chi\right)(x)=\int \frac{\partial^{M}}{\partial y_{1}}\left[\beta_{k}(x-y) u\left(N y_{1}-2 j\right) \chi\left(y^{\prime}\right)\right] \frac{e^{2 \pi i 2^{j} y_{1}}}{\left(-2 \pi i 2^{j}\right)^{M}} d y_{1} d y^{\prime}
$$

and thus, using that $N<2^{k}$,

$$
\left\|\beta_{k} *\left(g_{N, j} \otimes \chi\right)\right\|_{p} \lesssim 2^{-(j-k) M} N^{-1 / p}, \quad j \geq k .
$$

For $N / 8<j \leq k$ we use the cancellation of the $\beta_{k}$ (with $M$ vanishing moments) to obtain

$$
\left\|\beta_{k} *\left(g_{N, j} \otimes \chi\right)\right\|_{p} \lesssim 2^{-k M}\left\|\partial^{M} g_{N, j}\right\|_{\infty} N^{-\frac{1}{p}} \lesssim 2^{-(k-j) M} N^{-1 / p}, \quad j \leq k .
$$

Thus

$$
\begin{aligned}
& \left(\sum_{2^{k}>N} 2^{k q}\left\|\beta_{k} * f_{N}\right\|_{p}^{q}\right)^{\frac{1}{q}}=\left(\sum_{2^{k}>N} 2^{k q}\left[\sum_{\frac{N}{8}<j<\frac{N}{4}} 2^{-j p}\left\|\beta_{k} *\left(g_{N, j} \otimes \chi\right)\right\|_{p}^{p}\right]^{\frac{q}{p}}\right)^{\frac{1}{q}} \\
& \lesssim\left(\sum_{2^{k}>N}\left[\sum_{\frac{N}{8}<j<\frac{N}{4}} 2^{(k-j) p} 2^{-|j-k| M p}\right]^{\frac{q}{p}}\right)^{\frac{1}{q}} N^{-\frac{1}{p}} \lesssim N^{\frac{1}{q}-\frac{1}{p}}
\end{aligned}
$$


We now take $\Psi_{N}=2^{N d} \Psi\left(2^{N}\right.$. $)$ with $\Psi$ as in $\$ 5.1$, and we shall prove that

$$
\left\|\mathbb{E}_{N} f_{N}\right\|_{B_{p, \infty}^{1}} \gtrsim 2^{N}\left\|\Psi_{N} * \mathbb{E}_{N} f_{N}\right\|_{p} \gtrsim 1
$$

Define $\Theta: \mathbb{R} \rightarrow \mathbb{R}$ by

$$
\Theta(t)=\int_{-\infty}^{t} \theta(s) d s
$$

with $\theta=\phi_{0}^{(2 M)}$ as in $\$ 5.1$ and observe that $\Theta$ is odd, supported in $\left(-\frac{1}{8}, \frac{1}{8}\right)$ and $\int_{-\infty}^{\infty} \Theta(t) d t=0$. In particular,

$$
\int_{0}^{1 / 8}|\Theta(t)|^{p} d t \neq 0
$$

Let $\mathbb{E}_{N}^{(1)}$ and $\mathbb{E}_{N}^{(d-1)}$ be the dyadic averaging operators on $\mathbb{R}$ and $\mathbb{R}^{d-1}$, respectively. If we denote $\theta_{N}=2^{N} \theta\left(2^{N} \cdot\right), N \geq 1$, then we claim that

$$
\Psi_{N} *\left(\mathbb{E}_{N}\left[g_{N, j} \otimes \chi\right]\right)\left(x_{1}, x^{\prime}\right)=\theta_{N} *\left(\mathbb{E}_{N}^{(1)} g_{N, j}\right)\left(x_{1}\right), \text { for } x^{\prime} \in\left(\frac{1}{3}, \frac{2}{3}\right)^{d-1} \text {. }
$$

Indeed, for $x^{\prime} \in\left(\frac{1}{3}, \frac{2}{3}\right)^{d-1}$ it is easily seen that

$$
\begin{aligned}
& 2^{N(d-1)} \varphi_{0}\left(2^{N} \cdot\right) * \mathbb{E}_{N}^{(d-1)} \chi\left(x^{\prime}\right)=\int \varphi_{0}\left(y^{\prime}\right) d y^{\prime}=1, \\
& 2^{N(d-1)} \vartheta\left(2^{N} \cdot\right) * \mathbb{E}_{N}^{(d-1)} \chi\left(x^{\prime}\right)=\int \vartheta\left(y^{\prime}\right) d y^{\prime}=0 .
\end{aligned}
$$

The proof of the lower bound in (49) will rely on the following lemma.

Lemma 6.4. Let $\nu \in \mathbb{Z}$ and $\widetilde{I}_{N, \nu}=\left[\frac{\nu}{2^{N}}, \frac{\nu+1 / 8}{2^{N}}\right]$. Then, for every $t \in \widetilde{I}_{N, \nu}$ and $N / 8<j<N / 4$ we have

$$
\theta_{N} *\left(\mathbb{E}_{N}^{(1)} g_{N, j}\right)(t)=2^{-N} g_{N, j}^{\prime}\left(\frac{\nu}{2^{N}}\right) \Theta\left(2^{N} t-\nu\right)+O\left(2^{-2(N-j)}\right) .
$$

Moreover if $\frac{\nu}{2^{N}} \in\left[\frac{2 j}{N}+\frac{1}{4 N}, \frac{2 j}{N}+\frac{3}{4 N}\right]$ then $g_{N, j}^{\prime}\left(2^{-N} \nu\right)=2 \pi i 2^{j} e^{2 \pi i 2^{j-N} \nu}$.

Proof. The last assertion is immediate by the definition of $g_{N, j}$ in (44). So we focus in proving (53). We split

$$
\theta_{N} * \mathbb{E}_{N}^{(1)} g_{N, j}=\theta_{N} *\left(\mathbb{E}_{N}^{(1)}-I\right) g_{N, j}+\theta_{N} * g_{N, j}
$$

and observe that from the cancellation properties of $\theta_{N}$ we have

$$
\left\|\theta_{N} * g_{N, j}\right\|_{\infty} \lesssim 2^{-2 N}\left\|g_{N, j}^{\prime \prime}\right\|_{\infty} \lesssim 2^{-2 N}\left(2^{2 j}+N^{2}\right)
$$

which for $N / 8 \leq j \leq N / 4$ implies $\left\|\theta_{N} * g_{N, j}\right\|_{\infty} \lesssim 2^{-2(N-j)}$. Let $I_{N, \nu}=$ $\left[2^{-N} \nu, 2^{-N}(\nu+1)\right)$. For $t \in \widetilde{I}_{N, \nu}$ we have $\operatorname{supp} \theta_{N}(t-\cdot) \subset I_{N, \nu-1} \cup I_{N, \nu}$, so 
recalling (50) we obtain

$$
\begin{aligned}
\theta_{N} *\left(\mathbb{E}_{N}^{(1)}-I\right) g_{N, j}(t) & = \\
\int 2^{N} \Theta^{\prime}\left(2^{N}(t-s)\right) \times[ & \mathbb{1}_{I_{N, \nu}}(s)\left(f_{I_{N, \nu}} g_{N, j}(w) d w-g_{N, j}(s)\right) \\
& \left.+\mathbb{1}_{I_{N, \nu-1}}(s)\left(f_{I_{N, \nu-1}} g_{N, j}(w) d w-g_{N, j}(s)\right)\right] d s
\end{aligned}
$$

For $s \in I_{N, \nu}$, using Taylor expansions one sees that

$$
\begin{aligned}
& f_{I_{N, \nu}} g_{N, j}(w) d w-g_{N, j}(s) \\
& =f_{I_{N, \nu}} g_{N, j}^{\prime}(s)(w-s) d w+f_{I_{N, \nu}} \int_{0}^{1}(1-\sigma) g_{N, j}^{\prime \prime}(s+\sigma(w-s)) d \sigma(w-s)^{2} d w \\
& =g_{N, j}^{\prime}\left(\frac{\nu}{2^{N}}\right) \frac{1}{2\left|I_{N, \nu}\right|}\left[\left(\frac{\nu+1}{2^{N}}-s\right)^{2}-\left(\frac{\nu}{2^{N}}-s\right)^{2}\right]+O\left(2^{2 j-2 N}\right) \\
& =g_{N, j}^{\prime}\left(\frac{\nu}{2^{N}}\right)\left[\frac{\nu+1 / 2}{2^{N}}-s\right]+O\left(2^{2 j-2 N}\right) .
\end{aligned}
$$

Similarly, for $s \in I_{N, \nu-1}$,

$$
f_{I_{N, \nu-1}} g_{N, j}(w) d w-g_{N, j}(s)=g_{N, j}^{\prime}\left(\frac{\nu}{2^{N}}\right)\left[\frac{\nu-1 / 2}{2^{N}}-s\right]+O\left(2^{2 j-2 N}\right) .
$$

Hence for $t \in \widetilde{I}_{N, \nu}$ we have

$$
2^{N} \theta_{N} *\left(\mathbb{E}_{N}^{(1)}-I\right) g_{N, j}(t)=A_{1, j}(t)+A_{2, j}(t)+O\left(2^{-2(N-j)}\right)
$$

where

$$
A_{1, j}(t)=g_{N, j}^{\prime}\left(\frac{\nu}{2^{N}}\right) \int 2^{N} \Theta^{\prime}\left(2^{N}(t-s)\right) \frac{1}{2^{N+1}}\left(\mathbb{1}_{I_{N, \nu}}(s)-\mathbb{1}_{I_{N, \nu-1}}(s)\right) d s
$$

and

$$
A_{2, j}(t)=g_{N, j}^{\prime}\left(\frac{\nu}{2^{N}}\right) \int 2^{N} \Theta^{\prime}\left(2^{N}(t-s)\right)\left(\frac{\nu}{2^{N}}-s\right) d s .
$$

Integration by parts yields (for $t \in \widetilde{I}_{N, \nu}$ )

$$
A_{2, j}(t)=g_{N, j}^{\prime}\left(2^{-N} \nu\right) \int 2^{N} \Theta\left(2^{N}(s-t)\right) d s=0
$$

since $\int \Theta(s) d s=0$. To compute $A_{1, j}(t)$ we observe that

$$
\begin{aligned}
& \int 2^{N} \Theta^{\prime}\left(2^{N}(t-s)\right)\left(\mathbb{1}_{I_{N, \nu}}(s)-\mathbb{1}_{I_{N, \nu-1}}(s)\right) d s \\
& =\left[\int_{\frac{\nu}{2^{N}}}^{\frac{\nu+1}{2^{N}}}-\int_{\frac{\nu-1}{2^{N}}}^{\frac{\nu}{2^{N}}}\right] \frac{d}{d s}\left(-\Theta\left(2^{N}(t-s)\right) d s\right. \\
& =-\Theta\left(2^{N}\left(t-\frac{\nu+1}{2^{N}}\right)\right)+2 \Theta\left(2^{N}\left(t-\frac{\nu}{2^{N}}\right)\right)-\Theta\left(2^{N}\left(t-\frac{\nu-1}{2^{N}}\right)\right) .
\end{aligned}
$$


For $t \in \widetilde{I}_{N, \nu}$ we have $\Theta\left(2^{N}\left(t-2^{-N}(\nu \pm 1)\right)\right)=0$ and thus

$$
A_{1, j}(t)=2^{-N} g_{j}^{\prime}\left(\frac{\nu}{2^{N}}\right) \Theta\left(2^{N} t-\nu\right), \quad t \in \widetilde{I}_{N, \nu} .
$$

Inserting these expressions into (54) we are led to (53).

We may now complete the proof of (49). Using (52), and the fact that, by (47), the functions $\theta_{N} *\left(\mathbb{E}_{N}^{(1)} g_{N, j}\right)$ are supported in the disjoint intervals $J_{N, j}:=\frac{2 j}{N}+\left(-\frac{2}{N}, \frac{2}{N}\right)$, we have

$$
\begin{aligned}
& 2^{N}\left\|\Psi_{N} *\left(\mathbb{E}_{N} f_{N}\right)\right\|_{p} \gtrsim 2^{N}\left(\sum_{\frac{N}{8}<j<\frac{N}{4}}\left\|\theta_{N} * g_{N, j}\right\|_{L^{p}(\mathbb{R})}^{p} 2^{-j p}\right)^{1 / p} \\
& \gtrsim\left(\sum_{\frac{N}{8}<j<\frac{N}{4} \nu: \frac{\nu}{2^{N} \in J_{N, j}}}\left[\left|2^{-j} g_{N, j}^{\prime}\left(\frac{\nu}{2^{N}}\right)\right|^{p} \int_{\widetilde{I}_{N, \nu}}\left|\Theta\left(2^{N} t-\nu\right)\right|^{p} d t-\frac{c 2^{(j-N) p}}{2^{N}}\right]\right)^{1 / p}
\end{aligned}
$$

using the previous lemma in the last step. Since by (51)

$$
\int_{\widetilde{I}_{N, \nu}}\left|\Theta\left(2^{N} t-\nu\right)\right|^{p} d t=2^{-N} \int_{0}^{1 / 8}|\Theta(t)|^{p} d t \gtrsim 2^{-N}
$$

we obtain, for sufficiently large $N$,

$$
2^{N}\left\|\Psi_{N} *\left(\mathbb{E}_{N} f_{N}\right)\right\|_{p} \gtrsim\left(\sum_{\frac{N}{8}<j<\frac{N}{4}} \sum_{\nu: \frac{\nu}{2^{N}} \in J_{N, j}} 2^{-N}\left(1-c^{\prime} 2^{-p N / 2}\right)\right)^{1 / p} \gtrsim 1 .
$$

This completes the proof of (49), which together with (46) establishes Theorem 6.2, and therefore also Theorem 6.1.

\section{NeCESSARY CONDItions FOR BOUNDEDNESS When $s \leq 0$}

7.1. The case $1<p \leq \infty, s=1 / p-1, q>1$. In these cases the operator $\mathbb{E}_{N}$ is not bounded in $B_{p, q}^{1 / p-1}$ because characteristic functions of cubes do not belong to the dual space $\left(B_{p, q}^{1 / p-1}\right)^{*}=B_{p^{\prime}, q^{\prime}}^{1 / p^{\prime}}$; see 95 , This also applies when $p=\infty$, since $\left(b_{\infty, q}^{-1}\right)^{*}=B_{1, q^{\prime}}^{1}$; see [10, $\left.\S 1.1 .5\right]$.

7.2. The case $p=\infty, s=-1, q \leq 1$. We shall show

$$
\left\|\mathbb{E}_{N}\right\|_{b_{\infty, q}^{-1} \rightarrow B_{\infty, q}^{-1}} \gtrsim N \text {. }
$$

To prove this we argue by duality and first note that

$$
\left\|\mathbb{E}_{N}\right\|_{b_{1, \infty}^{1} \rightarrow B_{1, \infty}^{1}} \approx N \text {. }
$$

Indeed by Theorem 6.1 we have $\left\|\mathbb{E}_{N}\right\|_{B_{1, \infty}^{1} \rightarrow B_{1, \infty}^{1}} \approx N$ and the lower bound is obtained by testing $\mathbb{E}_{N}$ on the Schwartz-function $f_{N}$ as in (45) satisfying $\left\|f_{N}\right\|_{b_{1, \infty}^{1}}=\left\|f_{N}\right\|_{B_{1, \infty}^{1}} \lesssim N^{-1}$ and $\left\|\mathbb{E}_{N} f_{N}\right\|_{B_{1, \infty}^{1}} \gtrsim 1, c f$. (46), (49). 
To establish (55), since $\left\|\mathbb{E}_{N}\right\|_{b_{\infty, q}^{-1} \rightarrow B_{\infty, q}^{-1}} \geq\left\|\mathbb{E}_{N}\right\|_{b_{\infty, q}^{-1} \rightarrow B_{\infty, 1}^{-1}}$, by (156) it suffices to prove that

$$
\left\|\mathbb{E}_{N}\right\|_{b_{\infty, q}^{-1} \rightarrow B_{\infty, 1}^{-1}} \gtrsim\left\|\mathbb{E}_{N}\right\|_{b_{1, \infty}^{1} \rightarrow B_{1, \infty}^{1}} .
$$

We use that $\left(b_{\infty, q}^{-1}\right)^{*}=B_{1, \infty}^{1}$ for $q \leq 1$; see [15, 2.5.1/Remark 7]. Then for $f \in \mathcal{S}$

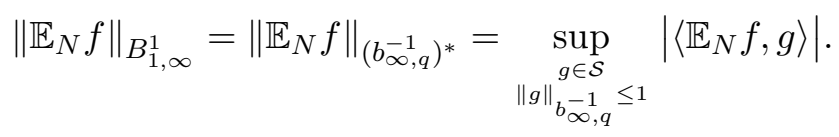

Now, for each $g \in \mathcal{S}$, since $f=\sum_{j=0}^{\infty} L_{j} \Lambda_{j} f$ in $\mathcal{S}$, we have

$$
\begin{aligned}
& \left|\left\langle\mathbb{E}_{N} f, g\right\rangle\right|=\left|\left\langle f, \mathbb{E}_{N} g\right\rangle\right| \leq \sum_{j=0}^{\infty}\left\|\Lambda_{j} f\right\|_{1}\left\|L_{j}\left(\mathbb{E}_{N} g\right)\right\|_{\infty} \\
& \lesssim\|f\|_{b_{1, \infty}^{1}}\left\|\mathbb{E}_{N} g\right\|_{B_{\infty, 1}^{-1}} \leq\|f\|_{b_{1, \infty}^{1}}\left\|\mathbb{E}_{N}\right\|_{b_{\infty, q}^{-1} \rightarrow B_{\infty, 1}^{-1}}\|g\|_{b_{\infty, q}^{-1}} .
\end{aligned}
$$

Inserting this into (58) we arrive at

$$
\left\|\mathbb{E}_{N} f\right\|_{B_{1, \infty}^{1}} \leq\left\|\mathbb{E}_{N}\right\|_{b_{\infty, q}^{-1} \rightarrow B_{\infty, 1}^{-1}}\|f\|_{b_{1, \infty}^{1}}
$$

and hence (57).

\section{Density And APproximation}

In this section we show two results regarding approximation by linear combinations of Haar functions. The main results in 88.1 are relevant to the formulation of Theorems 1.3 and 1.4 which rule out the case $s=1$. We shall also obtain a positive result about approximation for the spaces $b_{p, \infty}^{1 / p}$.

8.1. The case $s=1$. We shall show that no strongly admissible enumeration of the Haar system can form a Schauder basis on $B_{p, q}^{1}\left(\mathbb{R}^{d}\right)$ if $\frac{d}{d+1} \leq p<1$ and $q>0$. Moreover if in addition $0<q \leq p$ we shall show that the Haar system is not dense in $B_{p, q}^{1}\left(\mathbb{R}^{d}\right)$. It seems plausible that this last assertion would continue to hold for all $0<q \leq \infty$, but we do not have a proof in this generality.

Let us start with an auxiliary result. It is well-known that

$$
\|f\|_{B_{p, \infty}^{s}\left(\mathbb{R}^{d}\right)} \approx\|f\|_{p}+\sum_{j=1}^{d} \sup _{|h| \leq 1} \frac{\left\|\Delta_{h e_{j}}^{2} f\right\|_{p}}{\mid h^{s}},
$$

for all $p \leq 1$ and $d(1 / p-1)<s<2$, see [17, 2.6.1]. Below we show that a partial lower bound actually holds for all $0<s<2$, which allows to incorporate the endpoint $s=d(1 / p-1)=1$ (i.e., $p=d /(d+1)$ ) to our later results. 
Proposition 8.1. Let $0<s<2$ and $0<p \leq 1$. Then

$$
\|g\|_{p}+\sum_{j=1}^{d} \sup _{|h| \leq 1} \frac{\left\|\Delta_{h e_{j}}^{2} g\right\|_{p}}{|h|^{s}} \lesssim\|g\|_{B_{p, \infty}^{s}}
$$

holds for any function $g \in L^{1}\left(\mathbb{R}^{d}\right)$.

Proof. Let $\widehat{\psi}_{0} \in C_{c}^{\infty}\left(\mathbb{R}^{d}\right)$ supported in $\{|\xi|<3 / 8\}$ and with $\widehat{\psi}_{0}(\xi)=1$ if $|\xi| \leq 1 / 4$, and let $\widehat{\psi}_{k}(\xi)=\widehat{\psi}_{0}\left(2^{-k} \xi\right)-\widehat{\psi}_{0}\left(2^{-k+1} \xi\right)$ if $k \geq 1$. Consider a standard dyadic frequency decomposition $g=\sum_{k=0}^{\infty} g_{k}$, with $g_{k}=\psi_{k} * g$, which converges in $L^{1}$ and also a.e. Since

$$
\left(\sum_{k=0}^{\infty}\left\|g_{k}\right\|_{p}^{p}\right)^{1 / p} \lesssim\|g\|_{B_{p, \infty}^{s}}
$$

we also have $\|g\|_{p} \lesssim\|g\|_{B_{p, \infty}^{s}}$. In addition, for each $0<|h| \leq 1$, using the trivial estimate $\left\|\Delta_{h e_{j}}^{2} g_{k}\right\|_{p}^{p} \leq 4\left\|g_{k}\right\|_{p}^{p}$, we see that

$$
\begin{aligned}
\frac{\left\|\Delta_{h e_{j}}^{2} \sum_{2^{k} \geq|h|^{-1}} g_{k}\right\|_{p}}{|h|^{s}} & \leq\left(4 \sum_{2^{k} \geq|h|^{-1}}\left(2^{k}|h|\right)^{-s p}\left\|g_{k}\right\|_{p}^{p} 2^{k s p}\right)^{1 / p} \\
& \lesssim \sup _{2^{k}|h| \geq 1} 2^{k s}\left\|g_{k}\right\|_{p} .
\end{aligned}
$$

Let $\varphi \in \mathcal{S}$ be such that $\widehat{\varphi}(\xi)=1$ if $|\xi| \leq 1$. For every $0<v<1$, we let $K_{v e_{j}}=\Delta_{v e_{j}}^{2} \varphi$, so that

$$
\widehat{K}_{v e_{j}}(\xi)=\left(e^{2 \pi i\left\langle v e_{j}, \xi\right\rangle}-1\right)^{2} \hat{\varphi}(\xi) .
$$

Then $K_{v e_{j}}$ is a Schwartz function and we have the estimate

$$
\left|K_{v e_{j}}(x)\right| \leq C_{N} v^{2}(1+|x|)^{-2 N},
$$

for a large $N>d$. Hence, for each $k \geq 0$ such that $2^{k}|h|<1$, we have

$$
\begin{aligned}
& \left|\Delta_{h e_{j}}^{2} g_{k}(x)\right|=2^{k d}\left|K_{2^{k} h e_{j}}\left(2^{k} \cdot\right) * g_{k}(x)\right| \\
& \leq C_{N}\left(2^{k}|h|\right)^{2} \int 2^{k d}\left(1+2^{k}|y|\right)^{-2 N}\left|g_{k}(x-y)\right| d y \\
& \lesssim C_{N}\left(2^{k}|h|\right)^{2} \sup _{y \in \mathbb{R}^{d}}\left(1+2^{k}|y|\right)^{-N}\left|g_{k}(x-y)\right| .
\end{aligned}
$$

Choosing $N>d / p$ we can apply the Peetre maximal function estimate to obtain

$$
\begin{aligned}
& \frac{\left\|\Delta_{h e_{j}}^{2} \sum_{2^{k}<|h|^{-1}} g_{k}\right\|_{p}}{|h|^{s}} \leq\left(\sum_{2^{k}<|h|^{-1}}\left(2^{k}|h|\right)^{-s p}\left\|\Delta_{h e_{j}}^{2} g_{k}\right\|_{p}^{p} 2^{k s p}\right)^{1 / p} \\
& \lesssim\left(\sum_{2^{k}<|h|^{-1}}\left(2^{k}|h|\right)^{(2-s) p}\left\|g_{k}\right\|_{p}^{p} 2^{k s p}\right)^{1 / p} \lesssim \sup _{k \geq 0} 2^{k s}\left\|g_{k}\right\|_{p} .
\end{aligned}
$$

Combining the two estimates yields the result. 
Remark 8.2. The appropriate analogue for $B_{p, q}^{s}\left(\mathbb{R}^{d}\right)$-quasinorms for $q<\infty$, i.e.

$$
\|g\|_{p}+\sum_{j=1}^{d}\left(\int_{-1}^{1} \frac{\left\|\Delta_{h e_{j}}^{2} g\right\|_{p}^{q}}{|h|^{s q}} \frac{d h}{|h|}\right)^{1 / q} \lesssim\|g\|_{B_{p, q}^{s}}
$$

remains valid (when $0<s<2$ ) but is not relevant in this section.

The following proposition is a modification of our argument in 4, Proposition 4.2]. It shows the necessity of the condition $s<1$ in part (ii) of Theorem 1.3 and part (iii) of Theorem 1.4.

Proposition 8.3. There exists a Schwartz function $f$ supported in $\left(\frac{1}{16}, \frac{15}{16}\right)^{d}$ such that for all $0<p \leq 1$ it holds

$$
\liminf _{N \rightarrow \infty}\left\|\mathbb{E}_{N} f-f\right\|_{B_{p, \infty}^{1}}>0
$$

Proof. Let $\eta \in C_{c}^{\infty}\left(\mathbb{R}^{d}\right)$ be supported in $\left(\frac{1}{16}, \frac{15}{16}\right)^{d}$ with $\eta(x)=1$ if $x \in$ $(1 / 8,7 / 8)^{d}$. Let $f(x)=x_{1} \eta(x)$. From (59) we have

$$
\left\|\mathbb{E}_{N} f-f\right\|_{B_{p, \infty}^{1}} \gtrsim \sup _{0<h \leq 1} \frac{\left\|\Delta_{h e_{1}}^{2} \mathbb{E}_{N} f-\Delta_{h e_{1}}^{2} f\right\|_{p}}{h},
$$

Clearly, since $f$ is a Schwartz function,

$$
\left\|\Delta_{h e_{1}}^{2} f\right\|_{p} \lesssim|h|^{2}, \quad 0<h \leq 1
$$

So, by an appropriate triangle inequality, it suffices to show that

$$
\frac{\left\|\Delta_{2^{-N-2} e_{1}}^{2} \mathbb{E}_{N} f\right\|_{p}}{2^{-N-2}} \geq c>0
$$

for large $N$. We now recall a calculation in [4, Prop 4.2]. Let $N>10$ and let $h \in(0,1 / 4)$. An explicit calculation shows that for $x \in(1 / 4,3 / 4)^{d}$

$$
\mathbb{E}_{N} f(x)=\sum_{2^{N-2} \leq k<3 \cdot 2^{N-2}} \frac{k+1 / 2}{2^{N}} \mathbb{1}_{\left[\frac{k}{2^{N}}, \frac{k+1}{2^{N}}\right) \times[0,1)^{d-1}}(x) .
$$

Then, under the additional assumption $0<h<2^{-N-1}$,

$$
\Delta_{h e_{1}} \mathbb{E}_{N} f(x)=2^{-N-1} \sum_{2^{N-2} \leq k \leq 3 \cdot 2^{N-2}} \mathbb{1}_{\left[\frac{k}{2^{N}}-h, \frac{k}{2^{N}}\right) \times[0,1)^{d-1}}(x),
$$

and

$$
\begin{aligned}
& \Delta_{h e_{1}}^{2} \mathbb{E}_{N} f(x)= \\
& 2^{-N-1} \sum_{2^{N-2}<k<3 \cdot 2^{N-2}}\left(\mathbb{1}_{\left[\frac{k}{2^{N}}-2 h, \frac{k}{2^{N}}-h\right) \times[0,1)^{d-1}}(x)-\mathbb{1}_{\left[\frac{k}{2^{N}}-h, \frac{k}{2^{N}}\right) \times[0,1)^{d-1}}(x)\right) .
\end{aligned}
$$

Therefore,

and in particular

$$
\left\|\Delta_{h e_{1}}^{2} \mathbb{E}_{N} f\right\|_{L^{p}\left([0,1]^{d}\right)} \gtrsim 2^{N(1 / p-1)} h^{1 / p},
$$

$$
\left\|\Delta_{2^{-N-2} e_{1}}^{2} \mathbb{E}_{N} f\right\|_{L^{p}\left([0,1]^{d}\right)} \gtrsim 2^{-N}
$$


which implies (61).

Finally, we conclude with the non-density result mentioned above.

Corollary 8.4. Let $\frac{d}{d+1} \leq p<1,0<q \leq p$. Then $\operatorname{span} \mathcal{H}_{d}$ is not dense in $B_{p, q}^{1}\left(\mathbb{R}^{d}\right)$.

Proof. By Proposition 8.3 and $B_{p, q}^{1} \hookrightarrow B_{p, \infty}^{1}$ we have, for some $f \in C_{c}^{\infty}$,

$$
\liminf _{N \rightarrow \infty}\left\|\mathbb{E}_{N} f-f\right\|_{B_{p, q}^{1}}=c>0,
$$

By Theorem 1.8 the operators $\mathbb{E}_{N}$ are uniformly bounded on $B_{p, q}^{1}$. For $h \in \operatorname{span} \mathcal{H}_{d}$ we have $\mathbb{E}_{N} h=h$ for $N \geq N_{0}(h)$, with sufficiently large $N_{0}(h)$. Hence

$\left\|\mathbb{E}_{N} f-f\right\|_{B_{p, q}^{1}} \lesssim\left\|\mathbb{E}_{N}[f-h]\right\|_{B_{p, q}^{1}}+\|f-h\|_{B_{p, q}^{1}} \lesssim\|f-h\|_{B_{p, q}^{1}}$, for $N \geq N_{0}(h)$, and the density of $\operatorname{span} \mathcal{H}_{d}$ in $B_{p, q}^{s}$ would yield a contradiction to (62).

Remark 8.5. When $d /(d+1) \leq p<1$, it follows from Theorem 1.8, iv (or vi), and from the results in 99 below, that each strongly admissible enumeration $\mathcal{U}$ of $\mathcal{H}_{d}$ is a basic sequence for $B_{p, p}^{1}\left(\mathbb{R}^{d}\right)$, that is, $\mathcal{U}$ is a Schauder basis for the subspace

$$
\overline{\operatorname{span}}_{\mathcal{H}_{d}}{ }^{1}{ }_{p, p} .
$$

It may be of interest to identify this subspace. By Oswald's result in [8], it contains the class $\mathcal{B}_{p, p,(1)}^{1}\left(\mathbb{R}^{d}\right)$ defined by first order differences.

8.2. An approximation result for $b_{p, \infty}^{1 / p}$ when $1<p<\infty$. In the limiting case $s=1 / p$, recall that $\mathcal{H}_{d}$ is contained in $B_{p, q}^{1 / p}$ only if $q=\infty$. We show an approximation result in this case when $1<p<\infty$. Recall that $B_{p, \infty}^{s}$ is not separable, and that $b_{p, \infty}^{s}$ denotes the closure of $\mathcal{S}$ in $B_{p, \infty}^{s}$. Recall also, from Proposition [5.1, that $b_{p, \infty}^{1 / p} \cap \operatorname{span} \mathcal{H}_{d}=\{0\}$. However taking closures one obtains

Proposition 8.6. Let $1<p \leq \infty$. Then

$$
b_{p, \infty}^{1 / p}\left(\mathbb{R}^{d}\right) \subsetneq \overline{\operatorname{span}\left(\mathcal{H}_{d}\right)} B_{p, \infty}^{1 / p} .
$$

Proof. Let $1<p<\infty$. In view of [16, 2.5.12], we may use the equivalent norm

$$
\|f\|_{B_{p, \infty}^{1 / p}}=\|f\|_{p}+\sup _{h \neq 0} \frac{\left\|\Delta_{h} f\right\|_{p}}{|h|^{1 / p}} .
$$

By dimensional considerations it is clear that the characteristic function of any dyadic cube $I$ of side length $2^{-k}$ can be written as a unique linear combination of Haar functions of frequency at most $2^{k-1}$ supported in the 
dyadic unit cube containing $I$. It therefore suffices to show that for every $f \in C_{c}^{1}\left(\mathbb{R}^{d}\right)$ we have $\left\|f-f_{N}\right\|_{B_{p, \infty}^{1 / p}} \rightarrow 0$, where we choose

$$
f_{N}=\sum_{I \in \mathcal{D}_{N}} f\left(c_{I}\right) \mathbb{1}_{I}
$$

and $c_{I}$ denotes the center of $I$. Let $\mathcal{I}=\mathcal{I}(f)$ be the family of $I \in \mathcal{D}_{N}$ which intersect the support of $f$. Clearly for $f \in C_{c}^{1}$ we have

$$
\left\|f-f_{N}\right\|_{p} \leq \sqrt{d} 2^{-N} 2^{-N d / p}\left\|f^{\prime}\right\|_{\infty}(\# \mathcal{I}(f))^{1 / p} \lesssim f 2^{-N}
$$

so that $\left\|f-f_{N}\right\|_{p} \rightarrow 0$ for $N \rightarrow \infty$. For the main term it suffices to show that

$$
\sup _{h \neq 0} \frac{\left\|\Delta_{h}\left(f-f_{N}\right)\right\|_{p}}{|h|^{1 / p}} \lesssim 2^{-N\left(1-\frac{1}{p}\right)}
$$

and recall that we are assuming $p>1$.

For $j>N$ we define the sets

$$
\mathcal{U}_{N, j}=\left\{\left(y_{1}, \ldots, y_{d}\right) \in \mathbb{R}^{d}: \min _{1 \leq i \leq d} \operatorname{dist}\left(y_{i}, 2^{-N} \mathbb{Z}\right) \leq 2^{-j-1}\right\} .
$$

Assume that $2^{-j-2} \leq|h|_{\infty}<2^{-j-1}$, for some $j>N$. If $I \in \mathcal{D}_{N}$ then

$$
x \in I \backslash \mathcal{U}_{N, j} \text { implies } \quad x+h \in I,
$$

and thus $\Delta_{h} f_{N}(x)=0$. So we have

$$
\left\|\Delta_{h}\left(f-f_{N}\right)\right\|_{p}^{p}=A_{N}(h)+B_{N}(h)
$$

where

$$
\begin{aligned}
& A_{N}(h)=\int_{\mathcal{U}_{N, j}}\left|\Delta_{h}\left[f_{N}-f\right](x)\right|^{p} d x, \\
& B_{N}(h)=\int_{\mathcal{U}_{N, j}^{\complement}}|f(x+h)-f(x)|^{p} d x .
\end{aligned}
$$

In the second term we use $\left|\Delta_{h} f(x)\right| \leq|h| \int_{0}^{1}|\nabla f(x+s h)| d s$ to obtain $B_{N}(h) \leq\|\nabla f\|_{p}^{p}|h|^{p}$ and thus

$$
\sup _{|h|<2^{-N-2}} B_{N}(h) /|h| \lesssim_{f} 2^{-N(p-1)} .
$$

For the term $A_{N}(h)$ we use that $\left\|f-f_{N}\right\|_{\infty} \leq C_{f} 2^{-N}$, and also that $f$ is compactly supported, and obtain the estimate

$$
\begin{aligned}
A_{N}(h) & \lesssim \sum_{I \in \mathcal{D}_{N}} \int_{I \cap \mathcal{U}_{N, j}}\left|f(x+h)-f_{N}(x+h)\right|^{p}+\left|f(x)-f_{N}(x)\right|^{p} d x \\
& \lesssim f 2^{-N p} 2^{N} 2^{-j}
\end{aligned}
$$


since $\left|I \cap \mathcal{U}_{N, j}\right| \approx 2^{-j} 2^{-(d-1) N}$. Hence $\sup _{|h| \approx 2^{-j}} A_{N}(h) /|h| \lesssim_{f} 2^{-N(p-1)}$. Putting the two estimates together we get

$$
\sup _{|h|_{\infty} \leq 2^{-N-2}} \frac{\left\|\Delta_{h}\left(f-f_{N}\right)\right\|_{p}}{|h|^{1 / p}} \lesssim f 2^{-N\left(1-\frac{1}{p}\right)}
$$

Finally, if $|h| \gtrsim 2^{-N}$ we use (64) to have

$$
\sup _{|h|_{\infty} \geq 2^{-N}} \frac{\left\|\Delta_{h}\left(f-f_{N}\right)\right\|_{p}}{|h|^{1 / p}} \lesssim \frac{2\left\|f-f_{N}\right\|_{p}}{2^{-N / p}} \lesssim f 2^{-N\left(1-\frac{1}{p}\right)}
$$

This shows (65) and therefore $\left\|f-f_{N}\right\|_{B_{p, \infty}^{1 / p}} \rightarrow 0$ for $p>1$, completing the proof of the inclusion (63) when $p<\infty$. The case $p=\infty$ is immediate since for $f \in C_{c}^{1}$

$$
\left\|f-f_{N}\right\|_{B_{\infty, \infty}^{0}} \lesssim\left\|f-f_{N}\right\|_{\infty} \lesssim 2^{-N}
$$

by an elementary consideration. Finally, since $b_{p, \infty}^{1 / p}\left(\mathbb{R}^{d}\right)$ is closed in $B_{p, \infty}^{1 / p}\left(\mathbb{R}^{d}\right)$ Proposition 5.1 tells us that the inclusion (63) is proper.

Remark 8.7. When $0<p \leq 1$, the same proof gives a version of (65), namely

$$
\sup _{h \neq 0} \frac{\left\|\Delta_{h}\left(f-f_{N}\right)\right\|_{p}}{|h|^{s}} \lesssim 2^{-N(1-s)}, \quad \text { if } s<1
$$

This can be used similarly to show that $\mathcal{H}_{d}$ is dense in the space $b_{p, \infty}^{s}$ when $d(1 / p-1)<s<1$ and $d /(d+1)<p \leq 1$.

Remark 8.8. Since $B_{p, \infty}^{1 / p}$ is not separable, not every function $f \in B_{p, \infty}^{1 / p}$ can be approximated by Haar expansions in the norm topology. However, (local) weak* convergence does hold, with norm-uniformly bounded partial sums. More precisely, if $1<p \leq \infty$ and $\chi \in C_{c}^{\infty}\left(\mathbb{R}^{d}\right)$, then

$$
\left(f-S_{R}^{\mathcal{U}} f\right) \chi \stackrel{\mathrm{w}^{*}}{\longrightarrow} 0, \quad \text { and } \quad \sup _{R \geq 1}\left\|\chi S_{R}^{\mathcal{U}} f\right\|_{B_{p, \infty}^{1 / p}} \lesssim\|f\|_{B_{p, \infty}^{1 / p}}
$$

for all $f \in B_{p, \infty}^{1 / p}$ and any strongly admissible enumeration. This is a consequence of the duality relation $B_{p, \infty}^{1 / p}=\left(B_{p^{\prime}, 1}^{-1 / p^{\prime}}\right)^{*}$ and the (local) norm convergence of $S_{R}^{\mathcal{U}} g \rightarrow g$ in the $B_{p^{\prime}, 1}^{-1 / p^{\prime}}$ norm, when $g \in B_{p^{\prime}, 1}^{-1 / p^{\prime}}$, see Theorem 1.4. We thank the referee for raising the question of weak ${ }^{*}$ convergence.

\section{PARTial SUMS AND LOCALizATION}

9.1. Partial sums and strongly admissible enumerations. We shall use a partition of unity to make statements on the structure of the partial sum operators $S_{R}^{\mathcal{U}}$ associated with a strongly admissible enumeration $\mathcal{U}$.

Let $\varsigma \in C_{c}^{\infty}$ be supported in a $10^{-2}$ neighborhood of $[0,1)^{d}$ and so that

$$
\sum_{\nu \in \mathbb{Z}^{d}} \varsigma(\cdot-\nu) \equiv 1
$$

We shall denote $\varsigma_{\nu}=\varsigma(\cdot-\nu), \nu \in \mathbb{Z}^{d}$. 
In the sequel we will use the notation from Definition 1.2 and below. It is convenient to denote $\mathbb{E}_{-1}(g) \equiv 0$ and $T_{-1}[g, \mathfrak{a}]=\sum_{\mu \in \mathbb{Z}^{d}} a_{\mu}\left\langle g, h_{0, \mu}^{\overrightarrow{0}}\right\rangle h_{0, \mu}^{\overrightarrow{0}}$.

Lemma 9.1. Let $\mathcal{U}$ be a strongly admissible enumeration of $\mathcal{H}_{d}$. Then, for every $R \in \mathbb{N}$ and $\nu \in \mathbb{Z}^{d}$ there is an integer $N_{\nu}=N_{\nu}(R) \geq-1$ and sequences $\mathfrak{a}^{\kappa, \nu}, 0 \leq \kappa \leq b$, whose terms belong to $\{0,1\}$, such that for all locally integrable functions $g$ we have

$$
S_{R}^{\mathcal{U}}\left[g \varsigma_{\nu}\right]=\mathbb{E}_{N_{\nu}}\left[g \varsigma_{\nu}\right]+\sum_{\kappa=0}^{b} T_{N_{\nu}+\kappa}\left[g \varsigma_{\nu}, \mathfrak{a}^{\kappa, \nu}\right] .
$$

Proof. We write

$$
S_{R}^{\mathcal{U}}\left[g \varsigma_{\nu}\right]=\sum_{n=1}^{R} u_{n}^{*}\left(g \varsigma_{\nu}\right) u_{n}=\sum_{n=1}^{R} 2^{k(n) d}\left\langle g \varsigma_{\nu}, h_{k(n), \mu(n)}^{\epsilon(n)}\right\rangle h_{k(n), \mu(n)}^{\epsilon(n)} .
$$

Note that if $u_{n}^{*}\left(g \varsigma_{\nu}\right) \neq 0$ then necessarily $u_{n}$ is supported in $I_{\nu}^{* *}$. Let

$$
K_{\nu}=\max \left\{k(n): \operatorname{supp}\left(h_{k(n), \mu(n)}^{\epsilon(n)}\right) \subset I_{\nu}^{* *}, n=1, \ldots, R\right\} .
$$

If $K_{\nu} \leq b$ the asserted formula holds with $N_{\nu}=-1$. We therefore may assume $K_{\nu}>b$.

We let $n_{\nu}^{*} \in[1, R]$ such that $k\left(n_{\nu}^{*}\right)=K_{\nu}$. Now if $h_{k^{\prime}, \mu^{\prime}}^{\epsilon^{\prime}}$ is any other Haar function supported in $I_{\nu}^{* *}$ there is a unique $n^{\prime} \in \mathbb{N}$ such that $h_{k^{\prime}, \mu^{\prime}}^{\epsilon^{\prime}}=$ $h_{k\left(n^{\prime}\right), \mu\left(n^{\prime}\right)}^{\epsilon\left(n^{\prime}\right)}$. If in addition $k^{\prime} \leq K_{\nu}-b$ (in other words if for $u_{n^{\prime}}=h_{k\left(n^{\prime}\right), \mu\left(n^{\prime}\right)}^{\epsilon\left(n^{\prime}\right)}$ we have that $\left.\left|\operatorname{supp}\left(u_{n^{\prime}}\right)\right| \geq\left|\operatorname{supp}\left(u_{n_{\nu}^{*}}\right)\right| 2^{b}\right)$ then by the admissibility condition we must have $n^{\prime} \leq n_{\nu}^{*}$, in particular $n^{\prime} \leq R$. That means that all Haar functions with frequency $2^{k}$ and $k \leq K_{\nu}-b$ which are supported in $I_{\nu}^{* *}$ arise in the expansion (69). All other Haar functions that arise in this expansion have frequencies $2^{k}$ with $K_{\nu}-b+1 \leq k \leq K_{\nu}$. This establishes the assertion with $N_{\nu}=K_{\nu}-b+1$. The functions $\mathfrak{a}^{\kappa, \nu}$ defined on $\mathbb{Z}^{d} \times \Upsilon$ take values in $\{0,1\}$.

Remark. Formula (68) can be extended to all $g \in B_{p, q}^{s}$, when the indices $(s, p, q)$ are as in Theorems 1.8 and 1.9. In that case, one must interpret

$$
S_{R}^{\mathcal{U}}(g)=\sum_{j=0}^{\infty} S_{R}^{\mathcal{U}}\left(L_{j} \Lambda_{j} g\right)
$$

see Remarks 3.5 and 4.5 .

Proposition 9.2. Suppose that

$$
\sup _{N \geq 0}\left\|\mathbb{E}_{N}\right\|_{B_{p, q}^{s} \rightarrow B_{p, q}^{s}}+\sup _{\substack{N \geq-1 \\\|\mathfrak{a}\| \infty \leq 1}}\left\|T_{N}[\cdot, \mathfrak{a}]\right\|_{B_{p, q}^{s} \rightarrow B_{p, q}^{s}}<\infty
$$

Then, for every strongly admissible enumeration $\mathcal{U}$ and every cube $Q$ it holds

$$
\sup _{R \in \mathbb{N}} \operatorname{Op}\left(S_{R}^{\mathcal{U}}, B_{p, q}^{s}, Q\right)<\infty .
$$


Moreover, $\mathcal{U}$ is a local basic sequence of $B_{p, q}^{s}\left(\mathbb{R}^{d}\right)$, that is

$$
\lim _{R \rightarrow \infty}\left\|\chi \cdot\left(S_{R}^{\mathcal{U}} f-f\right)\right\|_{B_{p, q}^{s}}=0
$$

for all $\chi \in C_{c}^{\infty}\left(\mathbb{R}^{d}\right)$ and all $f \in{\overline{\operatorname{span} \mathcal{H}_{d}}}^{S}{ }^{s}$.

Proof. Using Lemma 9.1, the bound in (71) follows from (70). We now show the last assertion. Let $\chi \in C_{c}^{\infty}\left(\mathbb{R}^{d}\right)$ and $f \in \overline{\operatorname{span} \mathcal{H}_{d}} B_{p, q}^{s}$. Suppose that $\operatorname{supp} \chi \subset(-N, N)^{d}$, and pick any $\tilde{\chi} \in C_{c}^{\infty}$ such that $\tilde{\chi} \equiv 1$ in $[-N, N]^{d}$ and $\operatorname{supp} \tilde{\chi}$ contained in $Q:=(-2 N, 2 N)^{d}$. Observe that

$$
u_{n}^{*}(g)=u_{n}^{*}(\tilde{\chi} g), \quad \text { if } g \in B_{p, q}^{s} \text { and } \operatorname{supp} u_{n} \subset[-N, N]^{d},
$$

so we also have

$$
\chi \cdot S_{R}^{\mathcal{U}}[g]=\chi \cdot S_{R}^{\mathcal{U}}[\tilde{\chi} g], \quad \forall g \in B_{p, q}^{s} .
$$

Given $\varepsilon>0$, let $h \in \operatorname{span} \mathcal{H}_{d}$ be such that $\|f-h\|_{B_{p, q}^{s}}<\varepsilon /(1+A)$, with $A$ the constant in (71). Let $R_{0}=R_{0}(h)$ be such that $S_{R}^{\mathcal{U}}[h]=h$ for $R \geq R_{0}$. Then, for all such $R$ we have

$$
\begin{aligned}
\left\|\chi \cdot\left(S_{R}[f]-f\right)\right\|_{B_{p, q}^{s}} & =\left\|\chi \cdot\left(S_{R}[f-h]+h-f\right)\right\|_{B_{p, q}^{s}} \\
& \lesssim\left\|\chi \cdot S_{R}[\tilde{\chi}(f-h)]\right\|_{B_{p, q}^{s}}+\|\chi \cdot(h-f)\|_{B_{p, q}^{s}} \\
& \lesssim(A+1)\|f-h\|_{B_{p, q}^{s}}<\varepsilon,
\end{aligned}
$$

where in the second line we have used (74) with $g=f-h$.

9.2. Bourdaud localizations of Besov spaces. In the unbounded setting of $\mathbb{R}^{d}$, the $B_{p, q}^{s}$-norms do not satisfy "localization properties" when $p \neq q$; see e.g. the discussion in [10, p. 66]. At the endpoint cases considered here, this creates a difficulty when trying to derive 'global' Schauder basis properties from the local ones in the previous subsection. This difficulty is not present in the case of $F_{p, q}^{s}$ spaces; see [4, 6].

To handle this problem one may consider the class of $\ell^{p}$-local Besov spaces introduced by G. Bourdaud 2

$$
\left(B_{p, q}^{s}\right)_{\ell p}=\left\{f \in S^{\prime}:\|f\|_{\left(B_{p, q}^{s}\right)_{\ell p}}=\left[\sum_{\nu \in \mathbb{Z}^{d}}\|\varsigma(\cdot-\nu) \cdot f\|_{B_{p, q}^{s}}^{p}\right]^{1 / p}<\infty\right\}
$$

where $\varsigma \in C_{c}^{\infty}\left(\mathbb{R}^{d}\right)$ with $\sum_{\nu \in \mathbb{Z}^{d}} \varsigma(\cdot-\nu) \equiv 1$ as in (67). In [2] (see also [17, 2.4.7]) it is shown that this definition does not depend on the particular choice of $\varsigma$, and that $\left(B_{p, q}^{s}\right)_{\ell^{p}}=B_{p, q}^{s}$ if and only if $p=q$. Moreover one has the embeddings

$$
\begin{aligned}
& B_{p, q}^{s} \hookrightarrow\left(B_{p, q}^{s}\right)_{\ell^{p}} \text { if } 0<q \leq p, \\
& \left(B_{p, q}^{s}\right)_{\ell^{p}} \hookrightarrow B_{p, q}^{s} \text { if } p \leq q \leq \infty .
\end{aligned}
$$

Using this notation we can prove the following. 
Theorem 9.3. Let $s \in \mathbb{R}$ and $0<p, q \leq \infty$. Suppose that (70) holds. Then, every strongly admissible enumeration $\mathcal{U}$ of $\mathcal{H}_{d}$ is a basic sequence of $\left(B_{p, q}^{s}\right)_{\ell^{p}}$. Moreover, $\mathcal{U}$ is a Schauder basis of $\left(B_{p, q}^{s}\right)_{\ell^{p}}$ in each of the cases (i) to (iv) in Theorem 1.4.

Proof. For the first assertion it suffices to show that the operator norms of $S_{R} \equiv S_{R}^{\mathcal{U}}$ in $\left(B_{p, q}^{s}\right)_{\ell^{p}}$ are uniformly bounded in $R$. To do so we use the assumption (70), together with Lemma 9.1.

Observe first that $\varsigma_{\nu^{\prime}} S_{R}\left(f \varsigma_{\nu}\right)=0$ whenever $\left|\nu-\nu^{\prime}\right|_{\infty} \geq 3$. Hence

$$
\begin{aligned}
\left\|S_{R} f\right\|_{\left(B_{p, q}^{s}\right)_{\ell p}} & =\left(\sum_{\nu^{\prime}}\left\|\varsigma_{\nu^{\prime}} S_{R}\left(\sum_{\nu} \varsigma_{\nu} f\right)\right\|_{B_{p, q}^{s}}^{p}\right)^{\frac{1}{p}} \\
& \lesssim\left(\sum_{\nu^{\prime}} \sum_{\nu:\left|\nu-\nu^{\prime}\right|_{\infty} \leq 2}\left\|\varsigma_{\nu^{\prime}} S_{R}\left(f \varsigma_{\nu}\right)\right\|_{B_{p, q}^{s}}^{p}\right)^{1 / p} \\
& \lesssim\left(\sum_{\nu}\left\|S_{R}\left(f \varsigma_{\nu}\right)\right\|_{B_{p, q}^{s}}^{p}\right)^{1 / p}
\end{aligned}
$$

using in the last step that $\varsigma_{\nu^{\prime}}$ is a uniform multiplier in $B_{p, q}^{s}$; see [17, 4.2.2]. Then Lemma 9.1 and (70) give

$$
\begin{aligned}
\left\|S_{R} f\right\|_{\left(B_{p, q}^{s}\right)_{\ell p}} & \lesssim\left(\sum_{\nu}\left\|\mathbb{E}_{N_{\nu}}\left(f \varsigma_{\nu}\right)\right\|_{B_{p, q}^{s}}^{p}+\left\|\sum_{\kappa=0}^{b} T_{N_{\nu}}\left[f \varsigma_{\nu}, \mathfrak{a}^{\kappa, \nu}\right]\right\|_{B_{p, q}^{s}}^{p}\right)^{1 / p} \\
& \lesssim b \quad\left(\sum_{\nu}\left\|f \varsigma_{\nu}\right\|_{B_{p, q}^{s}}^{p}\right)^{1 / p}=\|f\|_{\left(B_{p, q}^{s}\right)_{\ell} p}
\end{aligned}
$$

This shows the first part. Also, the Schauder basis property will hold if and only if $\operatorname{span} \mathcal{H}_{d}$ is dense in $\left(B_{p, q}^{s}\right)_{\ell^{p}}$.

We now show that density holds in the range of Theorem 1.4. Since $p<\infty$, for each $f \in\left(B_{p, q}^{s}\right)_{\ell^{p}}$ and $\varepsilon>0$ there is some $g \in B_{p, q}^{s}$ with compact support such that $\|f-g\|_{\left(B_{p, q}^{s}\right)_{\ell p}}<\varepsilon$. Moreover, in the asserted range $\operatorname{span} \mathcal{H}_{d}$ is dense in $B_{p, q}^{s}$, so if $\operatorname{supp} g \subset(-N, N)^{d}=Q$, then by Proposition 9.2 we may find a sufficiently large $R$ such that $\left\|g-S_{R} g\right\|_{B_{p, q}^{s}}<\varepsilon /|Q|^{1 / p}$. Since also $\operatorname{supp}\left(S_{R} g\right) \subset Q$ we deduce that

$$
\left\|g-S_{R} g\right\|_{\left(B_{p, q}^{s}\right)_{\ell} p} \lesssim|Q|^{1 / p}\left\|g-S_{R} g\right\|_{B_{p, q}^{s}}<\varepsilon,
$$

which completes the proof.

Finally, we gather as a corollary the positive Schauder results in the original scale of $B_{p, q}^{s}$ spaces.

Corollary 9.4. Every strongly admissible enumeration $\mathcal{U}$ of $\mathcal{H}_{d}$ is a Schauder basis of $B_{p, q}^{s}\left(\mathbb{R}^{d}\right)$ in each of the cases (i), (ii), (iii) in Theorem 1.3.

Proof. When $q=p$ the result is a consequence of the identity $B_{p, p}^{s}=\left(B_{p, p}^{s}\right)_{\ell^{p}}$ and the previous theorem. This covers the case (iii) in Theorem 1.3. For 

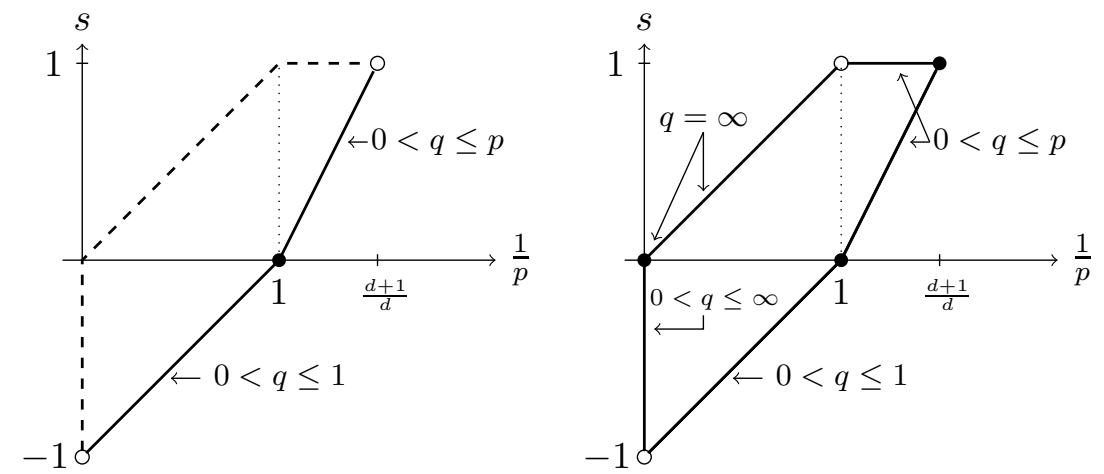

Figure 4. The left caption shows the region in which strongly admissible enumerations form a Schauder basis of the Bourdaud localization $\left(B_{p, q}^{s}\right)_{\ell^{p}} ;$ here always $q<\infty$. The right caption shows the corresponding region for the basic sequence property.

the other cases, in which $(1 / p, s)$ lies in the interior of the pentagon $\mathfrak{P}$, one proceeds by real interpolation as follows. Pick two numbers $s_{0}, s_{1}$ such that $s_{0}<s<s_{1}$ and $\left(1 / p, s_{i}\right) \in \mathfrak{P}, i=0,1$. Then, for some $\theta \in(0,1)$ we have

$$
B_{p, q}^{s}=\left(B_{p, p}^{s_{0}}, B_{p, p}^{s_{1}}\right)_{\theta, q}, \quad 0<q \leq \infty .
$$

Then the uniform boundedness of $S_{R}^{\mathcal{U}}$ on $B_{p, q}^{s}$ follows by interpolation from the diagonal cases.

9.3. Error estimates for compactly supported functions. Here we include a technical result related to localization which will be used in the proof of Theorem 10.2 below.

Let $f$ be supported in a dyadic cube $Q$ with sidelength $\ell(Q) \geq 1$. Since the function $\Lambda_{j} f$ does not have compact support, the terms $L_{k} \mathbb{E}_{N} L_{j} \Lambda_{j} f(x)$ will contribute for $x$ far away from the cube. We give a crude estimate which will suffice for our later application.

Let $\zeta \in C_{c}^{\infty}\left(\mathbb{R}^{d}\right)$ be supported on $(-2,2)^{d}$ and such that $\zeta \equiv 1$ on $\left[-\frac{3}{2}, \frac{3}{2}\right]^{d}$. If $y_{Q}$ is the center of $Q$, we define

$$
\zeta_{Q}(y)=\zeta\left(\left(y-y_{Q}\right) / \ell(Q)\right) .
$$

Clearly $\zeta_{Q} f=f$ for every distribution $f$ supported in $Q$. Moreover, this property continues to hold with $\zeta_{Q}$ replaced by $\tilde{\zeta}_{Q}$, where $\tilde{\zeta}(x)=\zeta(2 x)$. For $n \geq 1$ we let

$$
\zeta_{Q, n}(y)=\zeta\left(2^{-n}\left(y-y_{Q}\right) / \ell(Q)\right)-\zeta\left(2^{-n+1}\left(y-y_{Q}\right) / \ell(Q)\right) .
$$


Note that $\zeta_{Q, n}$ has support in $\left\{\frac{3}{4} \cdot 2^{n} \ell(Q)<\left|y-y_{Q}\right|_{\infty}<2^{n+1} \ell(Q)\right\}$, and that $\sum_{n \geq 1} \zeta_{Q, n} \equiv 1$.

Lemma 9.5. Let $s \leq 1,0<p \leq 1$ and $0<q \leq \infty$. Then, for every $M_{1}>1$ there exists a constant $C_{M_{1}}>0$ such that, if $f \in B_{p, q}^{s}\left(\mathbb{R}^{d}\right)$ is supported in a cube $Q$ with size $|Q| \geq 1$, then

$$
\left\|L_{k} \mathbb{E}_{N} L_{j}\left[\zeta_{Q, n} \Lambda_{j} f\right]\right\|_{p} \leq C_{M_{1}} 2^{-k / p} 2^{-j M_{1}} 2^{-n M_{1}}\|f\|_{B_{p, q}^{s}},
$$

for all $n \geq 1, k \geq 0, j \geq N$ and $N \geq 1$.

The same holds if $\mathbb{E}_{N}$ is replaced by $T_{N}[\cdot, \mathfrak{a}]$ with $\|\mathfrak{a}\|_{\infty} \leq 1$.

Proof. Let $\phi_{j}(x)=2^{j d} \phi\left(2^{j} x\right)$ be the convolution kernel of $\Lambda_{j}$, with $\phi \in \mathcal{S}$. Let

$$
F_{j, n}(x):=\zeta_{Q, n}(x) \Lambda_{j} f(x)=\zeta_{Q, n}(x)\left\langle\phi_{j}(x-\cdot) \tilde{\zeta}_{Q}(\cdot), f\right\rangle
$$

where we have used $f=f \tilde{\zeta}_{Q}$ for the second equation and the pairing $\langle\cdot, \cdot\rangle$ is in the sense of tempered distributions.

Pick a large $\gamma \in 2 \mathbb{N}$ such that $B_{p, q}^{s} \subset B_{2,2}^{-\gamma}$ (e.g., $\gamma>d\left(\frac{1}{p}-\frac{1}{2}\right)-s$ ). Then by duality

$$
\begin{aligned}
\left|F_{j, n}(x)\right| & \lesssim\left|\zeta_{Q, n}(x)\right|\left\|(I-\Delta)^{\gamma / 2}\left(\phi_{j}(x-\cdot) \tilde{\zeta}_{Q}(\cdot)\right)\right\|_{2}\|f\|_{B_{2,2}^{-\gamma}} \\
& \lesssim|Q|^{1 / 2} 2^{j(d+\gamma)}\left(1+2^{j+n} \ell(Q)\right)^{-M_{2}}\|f\|_{B_{p, q}^{s}} .
\end{aligned}
$$

Observe that $F_{j, n}$, and hence $L_{k} \mathbb{E}_{N} L_{j}\left[F_{j, n}\right]$, are all supported in a set of diameter $C 2^{n} \ell(Q)$. Then, if $k \leq N$ we have

$$
\begin{aligned}
\left\|L_{k} \mathbb{E}_{N} L_{j}\left(F_{j, n}\right)\right\|_{p} & \lesssim\left(2^{n d}|Q|\right)^{1 / p}\left\|L_{k} \mathbb{E}_{N} L_{j}\left(F_{j, n}\right)\right\|_{\infty} \\
& \lesssim\left(2^{n d}|Q|\right)^{1 / p}\left\|F_{j, n}\right\|_{\infty} .
\end{aligned}
$$

Inserting the bound (79) into this expression, with a sufficiently large $M_{2}$, and using that $k \leq N \leq j$, one easily obtains (78).

Assume now that $k>N$. We may use Proposition 2.2, i to obtain

$$
\left\|L_{k} \mathbb{E}_{N} L_{j}\left(F_{j, n}\right)\right\|_{p} \lesssim 2^{-\frac{k}{p}} 2^{j\left(\frac{d}{p}-d\right)} 2^{N\left(d-\frac{d-1}{p}\right)}\left\|\mathcal{M}_{j} F_{j, n}\right\|_{p} .
$$

By the support properties of $F_{j, n}$ we have

$$
\left\|\mathcal{M}_{j} F_{j, n}\right\|_{p} \lesssim\left(2^{n d}|Q|\right)^{1 / p}\left\|F_{j, n}\right\|_{\infty}
$$

so again, using (79) with a sufficiently large $M_{2}$, and the assumption $N \leq j$, one easily derives (78).

10. THE CASE $s=d\left(\frac{1}{p}-1\right)$ WHEN $q>p$.

In this section we restrict to the cases $q>p$ in the line $s=d / p-d$. We shall see that the individual operators $\mathbb{E}_{N}$ are not bounded, and hence positive results are not expected in this range.

Theorem 10.1. Let $0<p \leq 1$. If $q>p$ then the operators $\mathbb{E}_{N}$ are unbounded on $B_{p, q}^{d / p-d}\left(\mathbb{R}^{d}\right)$. 
We shall actually prove something stronger, namely optimal estimates for the local version of the operator norms $\mathrm{Op}\left(\mathbb{E}_{N}, B_{p, q}^{s}, Q\right)$ defined in (10). This may be of interest in the context of Besov spaces in bounded domains; see Remark 1.6. We remark that Oswald [8] also proved some lower bounds in a local setting which grow with $N$. The following theorem provides optimal growth rates.

Theorem 10.2. (i) If $0<p \leq 1$ and $p \leq q \leq \infty$, then there is a constant $c_{1}=c_{1}(p, q)>0$ so that

$$
\operatorname{Op}\left(\mathbb{E}_{N}, B_{p, q}^{d / p-d}, Q\right) \geq c_{1}\left(2^{N d}|Q|\right)^{\frac{1}{p}-\frac{1}{q}} .
$$

(ii) If in addition $\frac{d}{d+1} \leq p \leq q \leq 1$, then there is a constant $c_{2}=c_{2}(p, q)$, such that for any dyadic cube $Q$ with side length $\geq 1$ and any $N>10$

$$
c_{1} \leq \frac{\mathrm{Op}\left(\mathbb{E}_{N}, B_{p, q}^{d / p-d}, Q\right)}{\left(2^{N d}|Q|\right)^{\frac{1}{p}-\frac{1}{q}}} \leq c_{2} .
$$

Remark 10.3. From [16, 2.11.3] it is known that, when $0<p \leq 1$ and $1<q<\infty$, it holds

$$
\left(B_{p, q}^{d / p-d}\right)^{*}=B_{\infty, q^{\prime}}^{0}
$$

As $q^{\prime}<\infty$, this space does not contain the dual functionals $u_{n}^{*}$. In particular, the restriction in $q$ in part (ii) of Theorem 10.2 is natural, since for $q>1$ and $Q_{0}=(0,1)^{d}$ we have

$$
\operatorname{Op}\left(\mathbb{E}_{0}, B_{p, q}^{d / p-d}, Q_{0}\right)=\left\|\mathbb{1}_{Q_{0}}\right\|_{B_{p, q}^{d / p-d}}\left\|\mathbb{1}_{Q_{0}}\right\|_{\left(B_{p, q}^{d / p-d}\right)^{*}}=\infty ;
$$

see also [8, Thm 2.ii.a].

10.1. Proof of lower bounds in Theorem 10.2. We fix $0<p \leq 1$ and choose an positive integer $M>d / p-d$.

Let $\eta \in C_{c}^{\infty}(\mathbb{R})$ be an odd function, supported on $(-1 / 2,1 / 2)$, and such that $\int_{0}^{1 / 2} \eta(t) d t=1$ and $\int_{0}^{1 / 2} t^{n} \eta(t) d t=0$ for $n=1, \ldots, M$. Let further

$$
g_{l}\left(x_{1}, \ldots, x_{d}\right)=2^{l d} \prod_{i=1}^{d} \eta\left(2^{l} x_{i}\right)
$$

so that $\int g_{l}(x) P_{M}(x) d x=0$ whenever $P_{M}$ is a polynomial of degree $\leq M$. By the properties of $\eta$, if $l \geq N$ we have

$$
\mathbb{E}_{N}\left(g_{l}\right)(x)=2^{N d} \prod_{i=1}^{d}\left(\mathbb{1}_{\left[0,2^{-N}\right)}\left(x_{i}\right)-\mathbb{1}_{\left[-2^{-N}, 0\right)}\left(x_{i}\right)\right)=: h_{N}(x) .
$$

Notice that $h_{N}$ is not itself a Haar function, but up to a factor $(-2)^{d}$, it is a translate of a Haar function with Haar frequency $2^{N-1}$. Moreover, we also have

$$
\mathbb{E}_{N}\left[g_{l}(\cdot-\nu)\right]=h_{N}(\cdot-\nu), \quad \text { if } \nu \in 2^{-N} \mathbb{Z}^{d}, \quad l \geq N
$$


Let $\left\{\mathfrak{z}_{m}\right\}_{m=1}^{\infty}$ be an enumeration of $\mathbb{Z}^{d}$, and define

$$
f_{N}(x)=\sum_{m=1}^{\infty} a_{m} g_{N+m}\left(x-2^{-N+5} \mathfrak{z}_{m}\right) .
$$

Observe that the summands have disjoint supports. Also

$$
\mathbb{E}_{N} f_{N}=\sum_{m=1}^{\infty} a_{m} h_{N}\left(\cdot-2^{-N+5} \mathfrak{z}_{m}\right) .
$$

We claim that

$$
\left\|f_{N}\right\|_{B_{p, q}^{d / p-d}} \lesssim\left(\sum_{m=1}^{\infty}\left|a_{m}\right|^{q}\right)^{1 / q}
$$

and

$$
\left\|\mathbb{E}_{N} f_{N}\right\|_{B_{p, q}^{d / p-d}} \gtrsim\left(\sum_{m=1}^{\infty}\left|a_{m}\right|^{p}\right)^{1 / p} .
$$

This clearly implies that $\mathbb{E}_{N}$ cannot be a bounded operator on $B_{p, q}^{d / p-d}\left(\mathbb{R}^{d}\right)$ unless $q \leq p$.

We first show (86). To do so we construct specific functions $\Psi_{n}$ such that

$$
\|g\|_{B_{p, q}^{d / p-d}} \geq\|g\|_{B_{p, \infty}^{d / p-d}} \gtrsim \sup _{n \geq 1} 2^{n\left(\frac{d}{p}-d\right)}\left\|\Psi_{n} * f\right\|_{p}
$$

Let $\psi \in C_{c}^{\infty}(\mathbb{R})$ be supported in $(-1 / 2,1 / 2)$, with

$$
\int \psi(t) t^{l} d t=0, \quad l=0, \ldots, M,
$$

and such that, for some $\varepsilon>0$,

$$
\psi *\left(\mathbb{1}_{\left[0, \frac{1}{2}\right)}-\mathbb{1}_{\left[-\frac{1}{2}, 0\right)}\right)(t) \geq c>0 \quad \text { when } t \in\left[\frac{1}{2}, \frac{1}{2}+\varepsilon\right] .
$$

We then define

$$
\Psi_{n}(x)=2^{n d} \prod_{i=1}^{d} \psi\left(2^{n} x_{i}\right),
$$

which has enough vanishing moments to guarantee the validity of (87); see [17, 2.5.3]. In particular,

$$
\left\|\mathbb{E}_{N} f_{N}\right\|_{B_{p, q}^{d\left(\frac{1}{p}-1\right)}} \gtrsim 2^{N\left(\frac{d}{p}-d\right)}\left\|\Psi_{N+1} *\left(\mathbb{E}_{N} f_{N}\right)\right\|_{p}
$$

Next, using (88) one shows that, for $x \in 2^{-N+5} \mathfrak{z}_{m}+2^{-N-1}\left[\frac{1}{2}, \frac{1}{2}+\varepsilon\right]^{d}$,

$$
\Psi_{N+1} * h_{N}\left(x-2^{-N+5} \mathfrak{z} m\right) \geq 2^{N d} c^{d},
$$

and therefore

$$
\left\|\Psi_{N+1} * h_{N}\left(\cdot-2^{-N+5} \mathfrak{z}_{m}\right)\right\|_{p} \gtrsim 2^{N\left(d-\frac{d}{p}\right)} .
$$


Also the functions $\Psi_{N+1} * h_{N}\left(\cdot-2^{-N+5} \mathfrak{z}_{m}\right)$ have disjoint supports so that

$$
\begin{aligned}
\left\|\Psi_{N+1} * \mathbb{E}_{N} f_{N}\right\|_{p} & =\left(\sum_{m=1}^{\infty}\left|a_{m}\right|^{p}\left\|\Psi_{N+1} * h_{N}\left(\cdot-2^{-N+5} \mathfrak{z}_{m}\right)\right\|_{p}^{p}\right)^{1 / p} \\
& \gtrsim\left(\sum_{m}\left|a_{m}\right|^{p}\right)^{1 / p} 2^{-N d\left(\frac{1}{p}-1\right)}
\end{aligned}
$$

and (86) follows.

To prove (85) we examine $L_{j} g_{l}$ with $l=N+m$ and use the cancellation of the convolution kernel $\beta_{j}$ of $L_{j}$ when $j \geq l$, and the cancellation of $g_{l}$ for $j<l$. Here cancellation refers to $M$ vanishing moments. As a consequence we obtain the estimate

$$
\left|L_{j} g_{l}(x)\right| \lesssim \begin{cases}2^{l d} \mathbb{1}_{[-1,1]^{d}}\left(2^{l} x\right) 2^{-M|l-j|} & \text { for } j \geq l \\ 2^{j d_{1}} \mathbb{1}_{[-1,1]^{d}}\left(2^{j} x\right) 2^{-M|l-j|} & \text { for } j \leq l\end{cases}
$$

see a similar argument in the proof of [4, Lemma 2.2]. From here one easily obtains

$$
2^{j d\left(\frac{1}{p}-1\right)}\left\|L_{j} g_{l}\right\|_{p} \lesssim\left\{\begin{array}{ll}
2^{-\left(M-d\left(\frac{1}{p}-1\right)\right)|l-j|} & \text { if } j \geq l \\
2^{-M|l-j|} & \text { if } j \leq l
\end{array}\right\} \leq 2^{-\delta|l-j|},
$$

if we set $\delta=M-d\left(\frac{1}{p}-1\right)>0$. This leads to

$$
\begin{aligned}
2^{j d\left(\frac{1}{p}-1\right)}\left\|L_{j} f_{N}\right\|_{p} & \leq 2^{j d\left(\frac{1}{p}-1\right)}\left(\sum_{m=1}^{\infty}\left|a_{m}\right|^{p}\left\|L_{j} g_{N+m}\left(\cdot-2^{-N+5} \mathfrak{z}_{m}\right)\right\|_{p}^{p}\right)^{1 / p} \\
& \lesssim\left(\sum_{m=1}^{\infty}\left|a_{m}\right|^{p} 2^{-|N+m-j| \delta p}\right)^{1 / p}
\end{aligned}
$$

and consequently,

$$
\left(\sum_{j \geq 0}\left[2^{j d\left(\frac{1}{p}-1\right)}\left\|L_{j} f_{N}\right\|_{p}\right]^{q}\right)^{1 / q} \lesssim\left(\sum_{j \geq 0}\left(\sum_{m=1}^{\infty}\left|a_{m}\right|^{p} 2^{-|N+m-j| \delta p}\right)^{q / p}\right)^{1 / q} .
$$

Since $q \geq p$ we can apply the triangle inequality in $\ell^{q / p}$ to bound the previous expression, by

$$
\begin{aligned}
& \left(\sum_{j \geq 0}\left(\sum_{n \in \mathbb{Z}}\left|a_{n+j-N}\right|^{p} 2^{-|n| \delta p}\right)^{q / p}\right)^{1 / q} \\
& \lesssim\left(\sum_{j \geq 0} \sum_{n \in \mathbb{Z}}\left|a_{n+j-N}\right|^{q} 2^{-|n| q \frac{\delta}{2}}\right)^{1 / q} \lesssim\left(\sum_{m=1}^{\infty}\left|a_{m}\right|^{q}\right)^{1 / q} .
\end{aligned}
$$

This proves (85).

Finally, to establish the lower bound in Theorem 10.2, we simply chose

$$
a_{m}=\left\{\begin{array}{l}
1 \text { if } 2^{-N+5} \mathfrak{z}_{m} \in Q \\
0 \text { if } 2^{-N+5} \mathfrak{z}_{m} \notin Q .
\end{array}\right.
$$


Since $\left\{\mathfrak{z}_{m}\right\}$ enumerates $\mathbb{Z}^{d}$ and $\#\left(2^{-N+5} \mathbb{Z}^{d} \cap Q\right) \approx 2^{N d}|Q|$ we obtain

$$
\left\|f_{N}\right\|_{B_{p, q}^{d\left(\frac{1}{p}-1\right)}} \lesssim\left(2^{N d}|Q|\right)^{1 / q}
$$

from (85), and

$$
\left\|\mathbb{E}_{N} f_{N}\right\|_{B_{p, q}^{d\left(\frac{1}{p}-1\right)}} \gtrsim\left(2^{N d}|Q|\right)^{1 / p}
$$

from (86). This establishes the desired lower bound for all $q \geq p$.

10.2. Proof of upper bounds in Theorem 10.2 (ii). In what follows let $Q$ be a dyadic cube of side length $\geq 1$. We assume $\frac{d}{d+1} \leq p \leq q \leq 1$.

We use the global estimates (18), (19) and examine the two expressions on the right hand side of (19) corresponding to the cases $j \leq N$ and $j \geq N$. The terms for $j \leq N$ cause no problem. Namely, by Propositions 3.2 and 3.3 we have (for $p \leq q)$

$$
\left(\sum_{k=0} 2^{k\left(\frac{d}{p}-d\right) r}\left\|\sum_{j \leq N} L_{k} \mathbb{E}_{N}^{\perp} L_{j} \Lambda_{j} f\right\|_{p}^{r}\right)^{1 / r} \lesssim\|f\|_{B_{p, \infty}^{d / p-d}} \text { if } \frac{d}{d+1}<p \leq 1,
$$

and in the endpoint $q \geq p=\frac{d}{d+1}($ when $d / p-d=1)$ we have

$$
\begin{aligned}
\left(\sum_{k=0} 2^{k r}\left\|\sum_{j \leq N} L_{k} \mathbb{E}_{N}^{\perp} L_{j} \Lambda_{j} f\right\|_{p}^{r}\right)^{1 / r} & \\
& \lesssim\left(\sum_{j=0}^{N} 2^{j p}\left\|\Lambda_{j} f\right\|_{p}^{p}\right)^{1 / p} \lesssim N^{\frac{1}{p}-\frac{1}{q}}\|f\|_{B_{p, q}^{1}}, \quad p=\frac{d}{d+1}
\end{aligned}
$$

where we have applied Hölder's inequality. This global bound is far better than what is need for the conclusion and this part satisfies the target upper bound in (80).

Hence it suffices to prove, for $f$ supported in $Q$, the following bound

$$
\begin{aligned}
\left(\sum_{k=0}^{\infty} 2^{k d\left(\frac{1}{p}-1\right) r}\left\|\sum_{j \geq N+1} L_{k} \mathbb{E}_{N} L_{j} \Lambda_{j} f\right\|_{p}^{r}\right)^{1 / r} & \\
& \lesssim\left(2^{N d}|Q|\right)^{\frac{1}{p}-\frac{1}{q}}\left(\sum_{j=0}^{\infty} 2^{j d\left(\frac{1}{p}-1\right) q}\left\|\Lambda_{j} f\right\|_{p}^{q}\right)^{1 / q},
\end{aligned}
$$

for any $r>0$. Notice that Lemma 9.5 reduces matters to show the following inequalities.

$$
\begin{aligned}
\left(\sum_{k \geq N+1} 2^{k d\left(\frac{1}{p}-1\right) r}\left\|\sum_{j \geq N+1} L_{k} \mathbb{E}_{N} L_{j}\left[\zeta_{Q} \Lambda_{j} f\right]\right\|_{p}^{r}\right)^{1 / r} & \\
& \lesssim\left(2^{N d}|Q|\right)^{\frac{1}{p}-\frac{1}{q}}\left(\sum_{j=0}^{\infty} 2^{j d\left(\frac{1}{p}-1\right) q}\left\|\Lambda_{j} f\right\|_{p}^{q}\right)^{1 / q}
\end{aligned}
$$


and

$$
\begin{aligned}
\left(\sum_{k \leq N} 2^{k d\left(\frac{1}{p}-1\right) r}\left\|\sum_{j \geq N+1} L_{k} \mathbb{E}_{N} L_{j}\left[\zeta_{Q} \Lambda_{j} f\right]\right\|_{p}^{r}\right)^{1 / r} & \\
& \lesssim\left(2^{N d}|Q|\right)^{\frac{1}{p}-\frac{1}{q}}\left(\sum_{j=0}^{\infty} 2^{j d\left(\frac{1}{p}-1\right) q}\left\|\Lambda_{j} f\right\|_{p}^{q}\right)^{1 / q} .
\end{aligned}
$$

We first prove (94). Instead of using Proposition 3.1 directly we shall use a modification of its proof in [4, Proposition 2.1(i)]; we first recall some notation from that paper.

We let $\mathcal{D}_{N}$ be the collection of dyadic cubes of sidelength $2^{-N}$. For $j>N$ we define $\mathcal{U}_{N, j}$ as in (66), that is a $2^{-j-1}$-neighborhood of the set $\cup_{I \in \mathcal{D}_{N}} \partial I$. For $I \in \mathcal{D}_{N}$ and $l>N$ we denote by $\mathcal{D}_{l}[\partial I]$ the set of all $J \in \mathcal{D}_{l}$ such that $\bar{J} \cap \partial I \neq \emptyset$. Likewise, $\mathcal{D}_{N}(I)$ denotes the collection of cubes $I^{\prime} \in \mathcal{D}_{N}$ with $\bar{I} \cap \bar{I}^{\prime} \neq \emptyset$, that is the collection of neighboring cubes of $I$.

We use the following result taken from [4, Lemma 2.3].

Lemma 10.4. (i) Let $k>N \geq 1$ and $G$ be locally integrable. Then

$$
L_{k}\left(\mathbb{E}_{N} G\right)(x)=0, \quad \text { for all } x \in \mathcal{U}_{N, k}^{\complement}=\mathbb{R}^{d} \backslash \mathcal{U}_{N, k}
$$

(ii) Let $j>N \geq 1$, and $F$ locally integrable.

$$
\left|\mathbb{E}_{N}\left(L_{j} F\right)\right| \lesssim 2^{(N-j) d} \sum_{I \in \mathcal{D}_{N}} \sum_{J \in \mathcal{D}_{j+1}[\partial I]}\|F\|_{L^{\infty}(J)} \mathbb{1}_{I}
$$

Proof of (94). Observe that $F_{j}:=\zeta_{Q} \Lambda_{j} f$ and the functions $L_{k} \mathbb{E}_{N} L_{j}\left[F_{j}\right]$ are all supported in a fixed $C$-dilate of $Q$ (with say $C=10$ ). By Lemma 10.4, i, $L_{k} \mathbb{E}_{N}\left[L_{j} F_{j}\right](x)=0$ if $x \in \mathcal{U}_{N, k}^{\complement}$. We derive a pointwise estimate if $x \in \mathcal{U}_{N, k} \cap I$ for some $I \in \mathcal{D}_{N}$. From (97) and the fact that $\operatorname{supp} \beta_{k}(x-\cdot)$ is contained in the union of all $I^{\prime} \in \mathcal{D}_{N}(I)$ we have

$$
\begin{aligned}
\left|L_{k} \mathbb{E}_{N}\left[L_{j} F_{j}\right](x)\right| & \leq \int\left|\beta_{k}(x-y)\right|\left|\mathbb{E}_{N}\left(L_{j} F_{j}\right)(y)\right| d y \\
& \lesssim 2^{(N-j) d} \sum_{I^{\prime} \in \mathcal{D}_{N}(I)} \sum_{J \in \mathcal{D}_{j+1}\left[\partial I^{\prime}\right]}\left\|F_{j}\right\|_{L^{\infty}(J)}
\end{aligned}
$$


Let $Q_{*}$ be the above $C$-dilate of $Q$. Then using $\left|\mathcal{U}_{N, k} \cap I\right| \approx 2^{-N(d-1)-k}$ we have

$$
\begin{aligned}
& \left\|\sum_{j \geq N+1} L_{k} \mathbb{E}_{N}\left[L_{j} F_{j}\right]\right\|_{p} \\
& \lesssim\left(\sum_{\substack{I \in \mathcal{D}_{N} \\
I \cap Q_{*} \neq \emptyset}}\left|\mathcal{U}_{N, k} \cap I\right|\left|2^{N d} \sum_{j \geq N+1} 2^{-j d} \sum_{I^{\prime} \in \mathcal{D}_{N}(I)} \sum_{J \in \mathcal{D}_{j+1}\left[\partial I^{\prime}\right]}\left\|F_{j}\right\|_{L^{\infty}(J)}\right|^{p}\right)^{1 / p} \\
& \lesssim 2^{N d} 2^{-(N(d-1)+k) / p}\left(\sum_{\substack{I \in \mathcal{D}_{N} \\
I \cap Q_{*} \neq \emptyset}}\left|\sum_{j \geq N+1} 2^{-j d} \sum_{J \in \mathcal{D}_{j+1}[\partial I]}\left\|\Lambda_{j} f\right\|_{L^{\infty}(J)}\right|^{p}\right)^{1 / p} .
\end{aligned}
$$

The $I$-sum in the last display contains $\approx|Q| 2^{N d}$ terms. Let $p \leq q \leq 1$. Using Hölder's inequality in this sum we see that

$$
\begin{aligned}
& \left\|\sum_{j>N} L_{k} \mathbb{E}_{N}\left[L_{j} F_{j}\right]\right\|_{p} \lesssim\left(2^{N d}|Q|\right)^{\frac{1}{p}-\frac{1}{q}} \times \\
& 2^{N d_{2}} 2^{-(N(d-1)+k) / p}\left(\sum_{I \in \mathcal{D}_{N}}\left|\sum_{j>N} 2^{-j d} \sum_{J \in \mathcal{D}_{j+1}[\partial I]}\left\|\Lambda_{j} f\right\|_{L^{\infty}(J)}\right|^{q}\right)^{1 / q} .
\end{aligned}
$$

Consider the maximal function $\mathfrak{M}_{j} g(x)=\sup _{|h|_{\infty} \leq 2^{-j+1}}|g(x+h)|$. Then $\left\|\mathfrak{M}_{j} \Lambda_{j} f\right\|_{p} \lesssim c_{p}\left\|\Lambda_{j} f\right\|_{p}$ for all $p>0$. Moreover, as in [4, (22)], it holds

$$
\sup _{x \in J} \mathfrak{M}_{j} g(x) \lesssim\left[f_{J^{*}}\left|\mathcal{M}_{j} g(x+h)\right|^{p} d h\right]^{\frac{1}{p}},
$$

where $J^{*}$ is a $C^{\prime}$-dilate of the cube $J \in \mathcal{D}_{j+1}$. Therefore,

$$
\begin{aligned}
& \left(\sum_{I \in \mathcal{D}_{N}}\left|\sum_{j>N} 2^{-j d} \sum_{J \in \mathcal{D}_{j+1}[\partial I]}\left\|\Lambda_{j} f\right\|_{L^{\infty}(J)}\right|^{q}\right)^{1 / q} \\
& \lesssim\left(\sum_{I \in \mathcal{D}_{N}}\left|\sum_{j>N} 2^{-j d} \sum_{J \in \mathcal{D}_{j+1}[\partial I]} 2^{j d / p}\left\|\mathcal{M}_{j}\left[\Lambda_{j} f\right]\right\|_{L^{p}\left(J^{*}\right)}\right|^{q}\right)^{1 / q} .
\end{aligned}
$$

Using the embeddings $\ell^{p} \hookrightarrow \ell^{1}$ (for the $J$-sum) and $\ell^{q} \hookrightarrow \ell^{1}$ (for the $j$-sum), and in the second step $\ell^{p / q} \hookrightarrow \ell^{1}$ (for the $I$-sum), the above quantity is further estimated by

$$
\begin{aligned}
& \left(\sum_{I \in \mathcal{D}_{N}} \sum_{j>N} 2^{j d\left(\frac{1}{p}-1\right) q}\left(\sum_{J \in \mathcal{D}_{j+1}[\partial I]}\left\|\mathcal{M}_{j}\left[\Lambda_{j} f\right]\right\|_{L^{p}\left(J^{*}\right)}^{p}\right)^{q / p}\right)^{1 / q} \\
& \lesssim\left(\sum_{j>N} 2^{j d\left(\frac{1}{p}-1\right) q}\left(\sum_{I \in \mathcal{D}_{N}} \sum_{J \in \mathcal{D}_{j+1}[\partial I]}\left\|\mathcal{M}_{j}\left[\Lambda_{j} f\right]\right\|_{L^{p}\left(J^{*}\right)}^{p}\right)^{q / p}\right)^{1 / q} \\
& \lesssim\left(\sum_{j>N} 2^{j d\left(\frac{1}{p}-1\right) q}\left\|\mathcal{M}_{j}\left[\Lambda_{j} f\right]\right\|_{p}^{q}\right)^{1 / q} \lesssim\left(\sum_{j>N} 2^{j d\left(\frac{1}{p}-1\right) q}\left\|\Lambda_{j} f\right\|_{p}^{q}\right)^{1 / q} .
\end{aligned}
$$


Inserting this estimate into (98) we see that

$$
\begin{aligned}
& \left(\sum_{k>N} 2^{k d\left(\frac{1}{p}-1\right) r}\left\|\sum_{j>N} L_{k} \mathbb{E}_{N} L_{j}\left[\zeta_{Q} \Lambda_{j} f\right]\right\|_{p}^{r}\right)^{1 / r} \\
& \lesssim\left(2^{N d}|Q|\right)^{\frac{1}{p}-\frac{1}{q}}\left(\sum_{k>N} 2^{(N-k)\left(d-\frac{d-1}{p}\right) r}\right)^{1 / r}\left(\sum_{j>N} 2^{j d\left(\frac{1}{p}-1\right) q}\left\|\Lambda_{j} f\right\|_{p}^{q}\right)^{1 / q}
\end{aligned}
$$

and since the $k$-sum is $O(1)$ in the larger range $p>\frac{d-1}{d}$ we obtain (94) for $\frac{d}{d+1} \leq p \leq q \leq 1$.

Proof of (95). This case is simpler and can be obtained from the individual bounds of $\left\|L_{k} \mathbb{E}_{N} L_{j}\left[F_{j}\right]\right\|_{p}$ in Proposition 2.2 . Recall that $F_{j}=\zeta_{Q} \Lambda_{j} f$ and $L_{k} \mathbb{E}_{N} L_{j}\left[F_{j}\right]$ are supported in a $C$-dilate of $Q$.

Let $k \leq N$. Applying Hölder's inequality, the $q$-triangle inequality and Proposition 2.2. i we now obtain

$$
\begin{aligned}
2^{k s}\left\|\sum_{j>N} L_{k} \mathbb{E}_{N} L_{j}\left[F_{j}\right]\right\|_{p} \lesssim 2^{k s}|Q|^{\frac{1}{p}-\frac{1}{q}}\left[\sum_{j>N}\left\|L_{k} \mathbb{E}_{N} L_{j}\left[F_{j}\right]\right\|_{q}^{q}\right]^{\frac{1}{q}} \\
\lesssim|Q|^{\frac{1}{p}-\frac{1}{q}} 2^{k\left(s+d+1-\frac{d}{q}\right)} 2^{-N}\left(\sum_{j>N} 2^{j\left(\frac{d}{q}-d\right) q}\left\|\Lambda_{j} f\right\|_{q}^{q}\right)^{1 / q} .
\end{aligned}
$$

We now use the extension of Young's inequality

$$
\left\|\Lambda_{j} f\right\|_{q} \leq 2^{j\left(\frac{d}{p}-\frac{d}{q}\right)}\left\|\Lambda_{j} f\right\|_{p}
$$

see e.g. [16, 2.7.1/3]. As a result we obtain

$$
\begin{aligned}
2^{k d\left(\frac{1}{p}-1\right)}\left\|\sum_{j>N} L_{k} \mathbb{E}_{N} L_{j}\left[\zeta_{Q} \Lambda_{j} f\right]\right\|_{p} & \\
& \lesssim 2^{(k-N)\left(\frac{d}{p}-\frac{d}{q}+1\right)}\left(2^{N d}|Q|\right)^{\frac{1}{p}-\frac{1}{q}}\left(\sum_{j>N} 2^{j\left(\frac{d}{p}-d\right) q}\left\|\Lambda_{j} f\right\|_{p}^{q}\right)^{1 / q} .
\end{aligned}
$$

Finally, we may sum over $k \leq N$ using that $p \leq q$, and therefore obtain (95). With this assertion, the proof of Theorem 10.2 is now complete.

\section{A Strongly admissible EnUmeration}

We give explicit examples of strongly admissible enumerations for $\mathcal{H}_{d}$. We define the family of cubes

$$
\mathfrak{Q}_{5}=\left\{\prod_{i=1}^{d}\left[10 \kappa_{i}-5,10 \kappa_{i}+5\right): \kappa \in \mathbb{Z}^{d}\right\} .
$$

For $\ell=0,1,2, \ldots$, let $\mathfrak{Q}_{5}(\ell)$ be a strictly increasing collection of finite families of cubes from $\mathfrak{Q}_{5}$ such that for each cube in $\mathfrak{Q}_{5}(\ell)$ all its neighboring cubes in $\mathfrak{Q}_{5}$ belong to $\mathfrak{Q}_{5}(\ell+1)$, and such that $\mathfrak{Q}_{5}=\cup_{\ell} \mathfrak{Q}_{5}(\ell)$.

Example. We may take $\mathfrak{Q}_{5}(\ell)$ to be family of all $Q \in \mathfrak{Q}_{5}$ such that $Q \subset[-10 \ell-5,10 \ell+5)^{d}$. 
Let $\mathcal{A}_{0}=[-5,5)^{d}$, and for $\ell \geq 1$ let $\mathcal{A}_{\ell}$ be the union of cubes in $\mathfrak{Q}_{5}$ which belong to $\mathfrak{Q}_{5}(\ell) \backslash \mathfrak{Q}_{5}(\ell-1)$. For $\ell \geq 0$, let $\mathcal{H}_{d}(\ell, 0)$ be the family of characteristic functions of dyadic unit cubes contained in $\mathcal{A}_{\ell}$. For $k \geq 1$, $\ell \geq 0$, let $\mathcal{H}(\ell, k)$ be the family of Haar functions of mean value 0 and Haar frequency $2^{k-1}$ with the property that the interior of their support is contained in $\mathcal{A}_{\ell}$. Clearly, $\mathcal{H}_{d}=\cup_{\ell, k \geq 0} \mathcal{H}(\ell, k)$.

Let $N(\ell, k)=\# \mathcal{H}_{d}(\ell, k)$. We then have $N(0,0)=10^{d}$ and

$$
N(\ell, k)=N(\ell, 0) 2^{(k-1) d}\left(2^{d}-1\right) .
$$

In the specific example above the sets $\mathcal{A}_{\ell}, \ell \geq 1$, are corridors of width 10 , of the form $[-10 \ell-5,10 \ell+5)^{d} \backslash[-10 \ell+5,10 \ell-5)^{d}$ and we have $N(\ell, 0)=10^{d}\left((2 \ell+1)^{d}-(2 \ell-1)^{d}\right)$.

We now define an admissible enumeration $\mathcal{U}$ associated with this collection. Let $P(m)=\sum_{i=0}^{m} N(m-i, i)$, for $m=0,1,2, \ldots$, and let

$$
R(m)=\sum_{j=0}^{m} P(j)
$$

so that $R(m+1)-R(m)=P(m+1)$. First, for $n=1, \ldots, R(0)$ we enumerate the functions in $\mathcal{H}(0,0)$. Next, for $n=R(m)+1, \ldots, R(m+1)$ we enumerate the functions in $\cup_{i=0}^{m+1} \mathcal{H}(m+1-i, i)$ as follows: when

$$
R(m)+1 \leq n \leq R(m)+N(m+1,0)
$$

we enumerate the functions in $\mathcal{H}_{d}(m+1,0)$; subsequently, for each $\nu=$ $1, \ldots, m+1$, when

$$
R(m)+\sum_{i=0}^{\nu-1} N(m+1-i, i)+1 \leq n \leq R(m)+\sum_{i=0}^{\nu} N(m+1-i, i)
$$

we enumerate the functions in $\mathcal{H}_{d}(m+1-\nu, \nu)$.

That is, the functions in $\mathcal{H}\left(\ell^{\prime}, k^{\prime}\right)$ occur earlier than those in $\mathcal{H}(\ell, k)$ if $\ell^{\prime}+k^{\prime}<\ell+k$. Moreover, $\mathcal{H}(\ell+1, k-1)$ also occurs earlier than $\mathcal{H}(\ell, k)$. Now, if $u_{n}$ and $u_{n^{\prime}}$ are both supported in $I^{* *}$, the five-fold dilate of a fixed unit cube $I$, then their supports must be contained in cubes from $\mathcal{A}(\ell) \cup \mathcal{A}(\ell+1)$, for some smallest $\ell \geq 0$. Moreover, if $\left|\operatorname{supp} u_{n^{\prime}}\right| \geq 2^{d}\left|\operatorname{supp} u_{n}\right|$, that is, $k\left(n^{\prime}\right) \leq k(n)-1$, then the above observations imply that $u_{n^{\prime}}$ must occur before $u_{n}$. Thus, the enumeration we just constructed for $\mathcal{H}_{d}$ is strongly admissible with $b=1$.

In the next section it will be convenient to notice that, for the enumeration above, we have

$$
S_{R(m)} f=\mathbb{E}_{m-\ell} f, \quad \text { if } \operatorname{supp}(f) \subset \mathcal{A}_{\ell} \text { and } \ell \leq m .
$$

In particular we have $S_{R(m)} f=\mathbb{E}_{m} f$ if $f$ is supported in $(-5,5)^{d}$. 


\section{FAilure of CONVERGEnCE For Strongly ADMissible ENUMERATIONS}

In this section we prove the remaining negative results for the Schauder basis property, as stated in Theorem 1.3, namely the cases

(a) $s=\frac{d}{p}-d, \frac{d}{d+1} \leq p \leq 1$ and $0<q<p$

(b) $s=\frac{1}{p}-1,1<p<\infty$ and $0<q \leq 1$.

We remark that in these cases the operators $\mathbb{E}_{N}$ are uniformly bounded, by Theorem 1.8 (iii) and (vi), and local positive results hold by Theorem 9.3 . We disprove the possibility that the admissible enumerations in \$11 may be global Schauder bases in $B_{p, q}^{s}\left(\mathbb{R}^{d}\right)$. It suffices to show that the corresponding partial sum operators $S_{R}$ are not uniformly bounded.

12.1. The case $0<p \leq 1$.

Proposition 12.1. Let $0<q<p \leq 1$. Then, for the strongly admissible enumerations defined in $₫ 11$ we have

$$
\sup _{R \in \mathbb{N}} \sup \left\{\left\|S_{R} f\right\|_{B_{p, q}^{d\left(\frac{1}{p}-1\right)}}:\|f\|_{B_{p, q}^{d\left(\frac{1}{p}-1\right)}} \leq 1\right\}=\infty .
$$

Proof. We shall use a similar notation as in $\$ 10.1$. Consider functions $g_{l}$ as defined in (81). Fix $j \gg m$, and for $\ell \leq m$ pick $\mathfrak{z}_{\ell} \in \mathbb{Z}^{d}$ so that the threefold dilate of the cube $\mathfrak{z} \ell+[0,1)^{d}$ is contained in $\mathcal{A}_{\ell}$. Define

$$
f_{m, j}(x)=\sum_{\ell=1}^{m} g_{j}(x-\mathfrak{z} \ell) .
$$

Note that the summands $g_{j}(\cdot-\mathfrak{z} \ell)$ have disjoint supports in $\mathcal{A}_{\ell}$. By (101)

$$
S_{R(m)} f_{m, j}=\sum_{\ell=1}^{m} \mathbb{E}_{m-\ell}\left[g_{j}(\cdot-\mathfrak{z} \ell)\right]=\sum_{\ell=1}^{m} h_{m-\ell}(\cdot-\mathfrak{z} \ell),
$$

where $h_{N}$ was defined in (82).

Let $\Psi_{N}$ be defined as in (89), so that by (90) we have

$$
\left\|\Psi_{N+1} * h_{N}\right\|_{p} \gtrsim 2^{-N d\left(\frac{1}{p}-1\right)} \text {. }
$$

Then

$$
\begin{aligned}
\left\|S_{R(m)} f_{m, j}\right\|_{B_{p, q}^{d\left(\frac{1}{p}-1\right)}} \geq\left(\sum_{N=1}^{\infty} 2^{N\left(\frac{d}{p}-d\right) q}\left\|\Psi_{N+1} * S_{R(m)} f_{m, j}\right\|_{p}^{q}\right)^{1 / q} \\
=\left(\sum_{N=1}^{\infty} 2^{N\left(\frac{d}{p}-d\right) q}\left(\sum_{\ell=1}^{m}\left\|\Psi_{N+1} * h_{m-\ell}(\cdot-\mathfrak{z} \ell)\right\|_{p}^{p}\right)^{q / p}\right)^{1 / q} \\
\geq\left(\sum_{N=1}^{m-1} 2^{N\left(\frac{d}{p}-d\right) q}\left\|\Psi_{N+1} * h_{N}\right\|_{p}^{q}\right)^{1 / q} \gtrsim m^{1 / q} .
\end{aligned}
$$


Similarly, using the inequality in (92), that is

$$
2^{k d\left(\frac{1}{p}-1\right)}\left\|L_{k} g_{j}\right\|_{p} \lesssim 2^{-|j-k| \delta},
$$

for some $\delta>0$, we may conclude that

$$
\begin{aligned}
\left\|f_{m, j}\right\|_{B_{p, q}^{d\left(\frac{1}{p}-1\right)}} & \lesssim\left(\sum_{k=0}^{\infty} 2^{k d\left(\frac{1}{p}-1\right) q}\left\|L_{k}\left(f_{m, j}\right)\right\|_{p}^{q}\right)^{\frac{1}{q}} \\
& =\left(\sum_{k=0}^{\infty} 2^{k d\left(\frac{1}{p}-1\right) q}\left(\sum_{\ell=1}^{m}\left\|L_{k} g_{j}(\cdot-\mathfrak{z} \ell)\right\|_{p}^{p}\right)^{\frac{q}{p}}\right)^{\frac{1}{q}} \\
& \lesssim\left(\sum_{k=0}^{\infty} 2^{-|j-k| \delta q}\right)^{\frac{1}{q}} m^{1 / p} \lesssim m^{1 / p}
\end{aligned}
$$

Hence, the left hand side in (102) is $\gtrsim m^{1 / q-1 / p}$ which implies the assertion if $q<p$.

12.2. The case $1<p<\infty$. We shall deduce this case from the previous one. First of all notice that Proposition 12.1 remains to be valid when $1<p<\infty$. Indeed, the condition on $p$ did not play any role in the proof. In particular, if the dimension $d=1$ this implies

$$
\sup _{R \in \mathbb{N}}\left\|S_{R} f\right\|_{B_{p, q}^{\frac{1}{p}-1} \rightarrow B_{p, q}^{\frac{1}{p}-1}}=\infty, \quad \text { when } 0<q \leq 1<p .
$$

To establish the same result for $d \geq 2$, we tensorize the previous example. Consider

$$
F_{m, j}\left(x_{1}, x^{\prime}\right)=f_{m, j}\left(x_{1}\right) \chi\left(x^{\prime}\right),
$$

where $f_{m, j}$ is the 1-dimensional function in (103), and $\chi \in C_{c}^{\infty}\left((-2,2)^{d-1}\right)$ with $\chi \equiv 1$ in $[-1,1]^{d-1}$. We claim that, for $s=1 / p-1$ and $0<q \leq 1<p$, we have

$$
\left\|F_{m, j}\right\|_{B_{p, q}^{s}\left(\mathbb{R}^{d}\right)} \lesssim m^{1 / p}
$$

and

$$
\left\|S_{R(m, d)}\left(F_{m, j}\right)\right\|_{B_{p, q}^{s}\left(\mathbb{R}^{d}\right)} \gtrsim\left\|S_{R(m, 1)}\left(f_{m, j}\right)\right\|_{B_{p, q}^{s}(\mathbb{R})} \gtrsim m^{1 / q}
$$

Here $R(m, d)$ are the numbers in (100), where we stress the dependence on the dimension. Notice that in either case they verify (101).

To justify these inequalities, we construct a function $\Psi \in C_{c}^{\infty}\left(\mathbb{R}^{d}\right)$ as in (38), that is

$$
\Psi(x)=\Delta^{M}\left[\phi_{0} \otimes \varphi_{0}\right](x)=\theta\left(x_{1}\right) \varphi_{0}\left(x^{\prime}\right)+\phi_{0}\left(x_{1}\right) \vartheta\left(x^{\prime}\right),
$$

for suitable $\phi_{0}, \varphi_{0}, \theta, \vartheta$ as in the paragraph preceding (38). We let

$$
\Psi_{0}(x)=\phi_{0}\left(x_{1}\right) \varphi_{0}\left(x^{\prime}\right), \quad \text { and } \quad \Psi_{k}(x)=2^{k d} \Psi\left(2^{k} x\right), \quad k \geq 1 .
$$


These functions meet the required hypothesis to have

$$
\|g\|_{B_{p, q}^{s}\left(\mathbb{R}^{d}\right)} \approx\left(\sum_{k=0}^{\infty} 2^{k s q}\left\|\Psi_{k} * g\right\|_{L^{p}\left(\mathbb{R}^{d}\right)}^{q}\right)^{\frac{1}{q}} .
$$

Moreover, if we define, for $k \geq 1$,

$$
\phi_{k}\left(x_{1}\right)=2^{k} \theta\left(2^{k} x_{1}\right) \quad \text { and } \quad \varphi_{k}\left(x^{\prime}\right)=2^{(d-1) k} \vartheta\left(2^{k} x^{\prime}\right),
$$

then the convolutions with $\phi_{k}$ (respectively $\varphi_{k}$ ), $k=0,1,2, \ldots$, can be used to characterize the norms of $B_{p, q}^{s}$ in $\mathbb{R}$ (respectively in $\mathbb{R}^{d-1}$ ). Using this notation in (107) we can now write

$$
\Psi_{k}=\phi_{k} \otimes \varphi_{0, k}+\phi_{0, k} \otimes \varphi_{k},
$$

with $\phi_{0, k}\left(x_{1}\right)=2^{k} \phi_{0}\left(2^{k} x_{1}\right)$ and likewise for $\varphi_{0, k}$.

We now prove (106). First, using (101) one easily sees that

$$
S_{R(m, d)}\left(F_{m, j}\right)=\left(S_{R(m, 1)} f_{m, j}\right) \otimes\left(\mathbb{E}_{m}^{(d-1)}[\chi]\right) .
$$

Moreover, we claim that

$$
\Psi_{k} *\left(S_{R(m, d)} F_{m, j}\right)\left(x_{1}, x^{\prime}\right)=\phi_{k} *\left(S_{R(m, 1)} f_{m, j}\right)\left(x_{1}\right), \quad x^{\prime} \in\left(\frac{1}{4}, \frac{3}{4}\right)^{d-1} .
$$

Indeed, this is a direct consequence of (108) and

$$
\varphi_{0, k} *\left(\mathbb{E}_{m}^{(d-1)} \chi\right)\left(x^{\prime}\right)=\int \varphi_{0, k}=1 \quad \text { and } \quad \varphi_{k} *\left(\mathbb{E}_{m}^{(d-1)} \chi\right)\left(x^{\prime}\right)=\int \varphi_{k}=0 .
$$

Then (109) implies the first inequality in (106), and from the 1-dimensional result one obtains the second inequality.

We now prove (105). If $k \geq 1$ we can write

$$
\begin{aligned}
\Psi_{k} * F_{m, j} & =\left(\phi_{k} * f_{m, j}\right) \otimes\left(\varphi_{0, k} * \chi\right)+\left(\phi_{0, k} * f_{m, j}\right) \otimes\left(\varphi_{k} * \chi\right) \\
& =A_{k}+B_{k}
\end{aligned}
$$

(a similar formula holds for $k=0$ ). Then

$$
\left\|A_{k}\right\|_{p} \lesssim\left\|\phi_{k} * f_{m, j}\right\|_{p} \quad \text { and } \quad\left\|B_{k}\right\|_{p} \leq\left\|\phi_{0, k} * f_{m, j}\right\|_{p}\left\|\varphi_{k} * \chi\right\|_{p} .
$$

From the previous calculation in one dimension we have

$$
\left\|\phi_{k} * f_{m, j}\right\|_{p} \lesssim 2^{-|k-j| \delta} 2^{k\left(1-\frac{1}{p}\right)} m^{1 / p} .
$$

We estimate the term

$$
\left\|\phi_{0, k} * f_{m, j}\right\|_{p}=\left(\sum_{\ell=1}^{m}\left\|\phi_{0, k} * g_{j}\right\|_{p}^{p}\right)^{\frac{1}{p}}=m^{\frac{1}{p}}\left\|\phi_{0, k} * g_{j}\right\|_{p} .
$$

Now, if $k \geq j$ then

$$
\left\|\phi_{0, k} * g_{j}\right\|_{p} \leq\left\|\phi_{0, k}\right\|_{1}\left\|g_{j}\right\|_{p} \lesssim 2^{\left(1-\frac{1}{p}\right) j} \leq 2^{k\left(1-\frac{1}{p}\right)} .
$$

On the other hand, if $k<j$ then

$$
\left\|\phi_{0, k} * g_{j}\right\|_{p} \leq\left\|\phi_{0, k}\right\|_{p}\left\|g_{j}\right\|_{1} \lesssim 2^{k\left(1-\frac{1}{p}\right)} .
$$


Thus,

$$
\left\|B_{k}\right\|_{p} \lesssim m^{1 / p} 2^{k\left(1-\frac{1}{p}\right)}\left\|\varphi_{k} * \chi\right\|_{p}
$$

which can be inserted into (111), and overall will imply

$$
\left\|F_{m, j}\right\|_{B_{p, q}^{\frac{1}{p}-1}} \lesssim\left\|f_{m, j}\right\|_{B_{p, q}^{\frac{1}{p}-1}}+m^{\frac{1}{p}}\|\chi\|_{B_{p, q}^{0}} \lesssim m^{\frac{1}{p}}
$$

This completes the proof of (105), and hence of (104) for all $d>1$.

\section{Failure of unconditionality When $s=d / p-d$}

Theorem 1.3 states that strongly admissible enumerations of $\mathcal{H}_{d}$ form a Schauder basis of $B_{p, p}^{d / p-d}$ when $\frac{d}{d+1}<p \leq 1$. We show that the stronger conclusion of unconditionality fails. The argument will also apply to the Triebel-Lizorkin spaces $F_{p, q}^{d / p-d}$ and therefore we cover this case at the same time.

Theorem 13.1. For every $N \geq 1$, there is a collection $A(N)$ of Haar functions, all supported in $[0,1]^{d}$, with $\#(A(N)) \leq 2^{d} N$, and such that the orthogonal projection operators $P_{A(N)}$ satisfy the estimates

$$
\begin{aligned}
& \left\|P_{A(N)}\right\|_{B_{p, q}^{d\left(\frac{1}{p}-1\right)} \rightarrow B_{p, q}^{d\left(\frac{1}{p}-1\right)}} \gtrsim N^{1 / q}, \\
& \left\|P_{A(N)}\right\|_{F_{p, q}^{d\left(\frac{1}{p}-1\right)} \rightarrow F_{p, q}^{d\left(\frac{1}{p}-1\right)}} \gtrsim N^{1 / p} .
\end{aligned}
$$

We shall use the following well-known identity.

Lemma 13.2. For $N=1,2, \ldots$, it holds

$$
2^{N d} \mathbb{1}_{I_{N, 0}}=\mathbb{1}_{I_{0,0}}+\sum_{k=0}^{N-1} 2^{k d} \sum_{\epsilon \in \Upsilon} h_{k, 0}^{\epsilon} .
$$

Proof. The formula follows easily computing the Haar coefficients of the function on the left hand side of (112).

Let $F_{N}(x)=2^{N d} \mathbb{1}_{\left[0,2^{-N}\right)^{d}}(x)$, and let $G_{N}$ be its odd extension $G_{N}(x)=$ $F_{N}(x)-F_{N}(-x)$. Consider the finite dimensional subspace

$$
A(N)=\operatorname{span}\left(\left\{\mathbb{1}_{[0,1)^{d}}\right\} \cup \bigcup_{k=0}^{N-1}\left\{h_{k, 0}^{\epsilon}: \epsilon \in \Upsilon\right\}\right),
$$

which has dimension $\operatorname{dim} A(N)=\left(2^{d}-1\right) N+1$. Let $P_{A(N)}$ be the orthogonal projection onto $A(N)$. Then, by Lemma 13.2, $F_{N} \in A(N)$ and

$$
P_{A(N)}\left(G_{N}\right)=F_{N} \text {. }
$$

The failure of unconditionality follows now from 
Proposition 13.3. Let $\frac{d-1}{d}<p<\infty, q>0$. Then, for large $N$,

$$
\begin{aligned}
&\left\|G_{N}\right\|_{B_{p, q}^{\frac{d}{p}-d}} \lesssim 1, \\
&\left\|G_{N}\right\|_{F_{p, q}^{\frac{d}{p}-d}} \lesssim 1,
\end{aligned}
$$

and

$$
\begin{aligned}
& \left\|P_{A(N)} G_{N}\right\|_{B_{p, q}^{\frac{d}{p}-d}} \gtrsim N^{1 / q}, \\
& \left\|P_{A(N)} G_{N}\right\|_{F_{p, q}^{\frac{d}{p}-d}} \gtrsim N^{1 / p} .
\end{aligned}
$$

Proof. Since $\left\|\psi_{0} * G_{N}\right\|_{p} \lesssim 1$ for any $\psi_{0} \in \mathcal{S}$, we only need to estimate the terms involving $\psi_{k} * G_{N}$, with $k \geq 1$, in the $B_{p, q}^{s}$ or $F_{p, q}^{s}$ quasi-norms. Assume that $\psi_{k}(x)=2^{k d} \psi\left(2^{k} x\right)$, where $\psi \in C_{c}^{\infty}\left((-1,1)^{d}\right)$ is such that $\psi(x) \geq 1$ for $x \in(-1 / 2,-1 / 8)^{d}$, and $\psi$ has sufficient vanishing moments (to characterize the involved $\mathrm{B}$ and $\mathrm{F}$ norms). For $k \geq 1$, we analyze $\psi_{k} * G_{N}$. Note, that $G_{N}$ is supported on $\left[-2^{-N}, 2^{-N}\right]^{d}$. Since $\int G_{N}(x) d x=0$ we have

$$
\left|\psi_{k} * G_{N}(x)\right| \lesssim 2^{k d} 2^{k-N} \mathbb{1}_{\left[-2^{-k+1}, 2^{-k+1}\right]^{d}}, \quad \text { for } k \leq N
$$

see (91). Hence

$$
2^{k\left(\frac{d}{p}-d\right)}\left\|\psi_{k} * G_{N}\right\|_{p} \lesssim 2^{k-N}, \quad k \leq N
$$

For $k>N$ let $D_{N}$ be the boundary of $I_{N} \cup-I_{N}$. Then $\psi_{k} * G_{N}$ is supported in a $C 2^{-k}$ neighborhood $\mathcal{N}_{k, N}$ of $D_{N}$ and $\psi_{k} * G_{N}=O\left(2^{N d}\right)$ on $\mathcal{N}_{k, N}$. The measure of $\mathcal{N}_{k, N}$ is $O\left(2^{-N(d-1)-k}\right)$ and therefore we obtain

$$
2^{k\left(\frac{d}{p}-d\right)}\left\|\psi_{k} * G_{N}\right\|_{p} \lesssim 2^{-(k-N)\left(d-\frac{d-1}{p}\right)}, \quad k \geq N .
$$

Since $p>\frac{d-1}{d}$ we can sum the estimates and obtain (114a).

Similarly

$$
\begin{aligned}
& \left\|\left(\sum_{k=1}^{N}\left|\psi_{k} * G_{N}\right|^{q} 2^{k\left(\frac{d}{p}-d\right) q}\right)^{1 / q}\right\|_{p} \\
& \lesssim\left\|\left(\sum_{k=1}^{N}\left|2^{k d} 2^{k-N} \mathbb{1}_{|x| \lesssim 2^{-k}}\right|^{q} 2^{k\left(\frac{d}{p}-d\right) q}\right)^{1 / q}\right\|_{p} \lesssim 1
\end{aligned}
$$


and

$$
\begin{aligned}
& \left\|\left(\sum_{k=N+1}^{\infty}\left|\psi_{k} * G_{N}\right|^{q} 2^{k\left(\frac{d}{p}-d\right) q}\right)^{1 / q}\right\|_{p} \\
& \lesssim 2^{N d}\left\|\left(\sum_{k=N+1}^{\infty} 2^{k\left(\frac{d}{p}-d\right) q} \mathbb{1}_{\mathcal{N}_{k, N}}\right)^{1 / q}\right\|_{p} \\
& \lesssim 2^{N d}\left(\sum_{N \leq l<\infty} \operatorname{meas}\left(\mathcal{N}_{l, N}\right)\left(\sum_{N \leq k \leq l} 2^{k\left(\frac{d}{p}-d\right) q}\right)^{p / q}\right)^{1 / p} \\
& \lesssim 2^{N d}\left(\sum_{N \leq l<\infty} 2^{-l-N(d-1)}\left(\sum_{N \leq k \leq l} 2^{k\left(\frac{d}{p}-d\right) q}\right)^{p / q}\right)^{1 / p} \lesssim 1 .
\end{aligned}
$$

Observe that the last inequality requires a slightly different argument in each of the cases $\frac{d-1}{d}<p<1, p=1$ and $p>1$; we leave details to the reader. This proves (114b).

We now include the lower bound for $P_{A(N)} G_{N}=F_{N} \equiv 2^{N d} \mathbb{1}_{I_{N, 0}}$. Let

$$
\Omega_{k}=\left(-\frac{3 / 8}{2^{k}},-\frac{1 / 8}{2^{k}}\right)^{d} .
$$

Then, for $4 \leq k \leq N-4$,

$$
\psi_{k} * F_{N}(x)=\int 2^{k d} \psi\left(2^{k}(x-y)\right) 2^{N d} \mathbb{1}_{I_{N, 0}}(y) d y \geq 2^{k d}, \quad \text { for } x \in \Omega_{k},
$$

due to $2^{k}\left(x-\left[0,2^{-N}\right]^{d}\right) \subset(-1 / 2,-1 / 8)^{d}$ and the assumptions on $\psi$. Hence

$$
2^{k\left(\frac{d}{p}-d\right)}\left\|\psi_{k} * F_{N}\right\|_{p} \gtrsim 1, \quad 4 \leq k \leq N-4,
$$

which implies (115a). Also

$$
\left\|F_{N}\right\|_{F_{p, q}^{\frac{d}{p}-d}} \gtrsim\left(\sum_{k=4}^{N-4} \int_{\Omega_{k}} 2^{k\left(\frac{d}{p}-d\right) p} 2^{k d p}\right)^{1 / p} \gtrsim N^{1 / p}
$$

and (115b) follows.

14. Failure of unconditionality When $s=1 / p-1,1<p<\infty$.

In dimension $d=1$ the failure of unconditionality of $\mathcal{H}$ in $B_{p, q}^{\frac{1}{p}-1}(\mathbb{R})$ is already contained in Proposition 13.3. As happened in \$12.2, the argument for $d \geq 2$ requires a slight variation of the above.

We consider the finite dimensional space

$$
\mathcal{A}(N):=\operatorname{span}\left\{h \otimes \mathbb{1}_{[0,1]^{d-1}}: \quad h \in A^{(1)}(N)\right\},
$$

where $A^{(1)}(N)$ is the subspace defined in (113) (when $d=1$ ). Note that $\operatorname{dim} \mathcal{A}(N)=\operatorname{dim} A^{(1)}(N) \approx N$. We now have 
Theorem 14.1. Let $1<p<\infty$. Then

$$
\left\|P_{\mathcal{A}(N)}\right\|_{B_{p, q}^{\frac{1}{p}-1} \rightarrow B_{p, q}^{\frac{1}{p}-1}} \gtrsim N^{1 / q} .
$$

In particular, $\mathcal{H}^{d}$ is not unconditional in $B_{p, q}^{\frac{1}{p}-1}\left(\mathbb{R}^{d}\right)$ for any $q>0$.

Proof. We keep the notation $F_{N}$ and $G_{N}$ for the 1-dimensional functions in Proposition 13.3. We fix $\chi \in C_{c}^{\infty}\left((-1,2)^{d-1}\right)$ with $\chi \equiv 1$ in $[0,1]^{d-1}$, and define

$$
g_{N}(x)=G_{N}\left(x_{1}\right) \chi\left(x^{\prime}\right) \quad \text { and } \quad f_{N}(x)=F_{N}\left(x_{1}\right) \mathbb{1}_{[0,1]^{d-1}}\left(x^{\prime}\right) .
$$

Observe that $f_{N} \in \mathcal{A}(N)$ and

$$
P_{\mathcal{A}(N)}\left(g_{N}\right)=f_{N}
$$

by our choice of $\chi$. So, it suffices to show that, for large $N$,

$$
\left\|g_{N}\right\|_{B_{p, q}^{\frac{1}{p}-1}} \lesssim 1 \text { and }\left\|f_{N}\right\|_{B_{p, q}^{\frac{1}{p}-1}} \gtrsim N^{1 / q} .
$$

The first assertion is proved as in $\$ 12.2$, namely, one constructs functions $\Psi_{k}$ as in (108) and observes that

$$
\begin{aligned}
\Psi_{k} * g_{N} & =\left(\phi_{k} * G_{N}\right) \otimes\left(\varphi_{0, k} * \chi\right)+\left(\phi_{0, k} * G_{N}\right) \otimes\left(\varphi_{k} * \chi\right) \\
& =A_{k}+B_{k} .
\end{aligned}
$$

A similar proof as the one following (111) gives

$$
\left\|A_{k}\right\|_{p} \lesssim\left\|\phi_{k} * G_{N}\right\|_{p} \quad \text { and } \quad\left\|B_{k}\right\|_{p} \lesssim 2^{k\left(1-\frac{1}{p}\right)}\left\|\varphi_{k} * \chi\right\|_{p} .
$$

From here and the 1-dimensional results in (114a) it follows that

$$
\left\|g_{N}\right\|_{B_{p, q}^{\frac{1}{p}-1}\left(\mathbb{R}^{d}\right)} \lesssim\left\|G_{N}\right\|_{B_{p, q}^{\frac{1}{p}-1}(\mathbb{R})}+\|\chi\|_{B_{p, q}^{0}\left(\mathbb{R}^{d-1}\right)} \lesssim 1 .
$$

Likewise, to prove the second assertion in (117) one uses

$$
\Psi_{k} * f_{N}\left(x_{1}, x^{\prime}\right)=\phi_{k} * F_{N}\left(x_{1}\right), \quad x^{\prime} \in\left(\frac{1}{4}, \frac{3}{4}\right)^{d-1} .
$$

This identity, as before, follows from (108) and the facts

$$
\varphi_{0, k} * \mathbb{1}_{[0,1]^{d-1}}\left(x^{\prime}\right)=\int \varphi_{0, k}=1 \quad \text { and } \quad \varphi_{k} * \mathbb{1}_{[0,1]^{d-1}}\left(x^{\prime}\right)=\int \varphi_{k}=0,
$$

because the supports of $\varphi_{0, k}(x-\cdot)$ and $\varphi_{k}(x-\cdot)$ are contained in $[0,1]^{d-1}$ for such values of $x^{\prime}$. Thus,

$$
\left\|f_{N}\right\|_{B_{p, q}^{\frac{1}{p}-1}\left(\mathbb{R}^{d}\right)} \gtrsim\left\|F_{N}\right\|_{B_{p, q}^{\frac{1}{p}-1}(\mathbb{R})} \gtrsim N^{1 / q} .
$$

This establishes (117) and completes the proof of Theorem 14.1. 


\section{REFERENCES}

[1] F. Albiac, J.L. Ansorena, P. Berná, P. Wojtaszczyk. Greedy approximation for biorthogonal systems in quasi-Banach spaces. Preprint 2019, arXiv:1903.11651

[2] G. Bourdaud, Localisations des espaces de Besov. Studia Math. 90 (1988), 153-163.

[3] J. Franke, On the spaces $F_{p, q}^{s}$ of Triebel-Lizorkin type: Pointwise multipliers and spaces on domains. Math. Nachr. 125, 29-68 (1986).

[4] G. Garrigós, A. Seeger, T. Ullrich, The Haar system as a Schauder basis in spaces of Hardy-Sobolev type, Journal of Fourier Analysis and Applications, 24(5) (2018), 1319-1339.

[5] - On uniform boundedness of dyadic averaging operators in spaces of HardySobolev type. Analysis Math. 43 (2) (2017), 267-278.

[6] - The Haar system in Triebel-Lizorkin spaces: Endpoint results. Preprint, 2019, arXiv:1907.03738.

[7] P. Oswald, On inequalities for spline approximation and spline systems in the space $L_{p},(0<p<1)$, in Approximation and Function Spaces, Proc. Int. Conf. Gdansk 1979 (Z. Ciesielski, ed.), PWN Warszawa/North-Holland Amsterdam, pp. 531-552, 1981.

[8] $ـ$ Haar system as Schauder basis in Besov spaces: The limiting cases for $0<$ $p \leq 1$, arXiv e-print: arXiv:1808.08156

[9] J. Peetre, On spaces of Triebel-Lizorkin type. Ark. Mat. 13 (1975),123-130.

[10] T. Runst, W. Sickel, Sobolev spaces of fractional order, Nemytskij operators, and nonlinear partial differential equations, volume 3 of de Gruyter Series in Nonlinear Analysis and Applications. Walter de Gruyter \& Co., Berlin, 1996.

[11] A. Seeger, T. Ullrich, Haar projection numbers and failure of unconditional convergence in Sobolev spaces. Math. Z. 285 (2017), 91 - 119.

[12] Lower bounds for Haar projections: Deterministic Examples. Constr. Appr. 46 (2017), 227-242.

[13] H. Triebel. Über die Existenz von Schauderbasen in Sobolev-Besov-Räumen. Isomorphiebeziehungen. Studia Math. 46 (1973), 83-100.

[14] _ On Haar bases in Besov spaces. Serdica 4 (1978), no. 4, 330-343.

[15] _ Spaces of Besov-Hardy-Sobolev type. Teubner, Leipzig, 1978.

[16] - Theory of function spaces. Monographs in Mathematics, 78. Birkhäuser Verlag, Basel, 1983.

[17] — Theory of function spaces II. Monographs in Mathematics, 84. Birkhäuser Verlag, Basel, 1992.

[18] _ Function spaces in Lipschitz domains and on Lipschitz manifolds. Characteristic functions as pointwise multipliers. Rev. Mat. Complut. 15 (2002), no. 2, $475-524$.

[19] _ Bases in function spaces, sampling, discrepancy, numerical integration. EMS Tracts in Mathematics, 11. European Mathematical Society (EMS), Zürich, 2010.

[20] W. Yun, W. Sickel, D. Yang. Regularity Properties of the Haar System with Respect to Besov-type Spaces. Preprint arXiv:1898.09583. 
Gustavo Garrigós, Department of Mathematics, University of Murcia, 30100 Espinardo, Murcia, Spain

E-mail address: gustavo.garrigos@um.es

Andreas Seeger, Department of Mathematics, University of Wisconsin, 480 Lincoln Drive, Madison, WI,53706, USA

E-mail address: seeger@math.wisc.edu

Tino Ullrich, Faculty of Mathematics, Technical University of Chemnitz, 09107 Chemnitz, Germany

E-mail address: tino.ullrich@mathematik.tu-chemnitz.de 